\title{
Supporting Information Theoretical Study of Divalent Bis(Pentaisopropylcyclopentadienyl) Actinocenes
}

\author{
Jason M. Yu, Filipp Furche* \\ *ffurche@uci.edu
}

Department of Chemistry, University of California Irvine, 1102 Natural Sciences II, Irvine, CA 92697-2025, USA 


\section{Contents}

$\begin{array}{lll}1 & \text { Optimized coordinates for } \mathrm{An}\left(\mathrm{Cp}^{\mathrm{iPr}}\right)_{2} & \text { S3 }\end{array}$

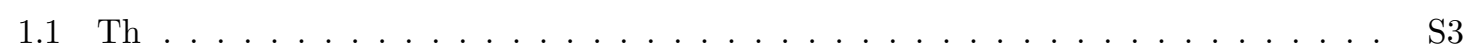

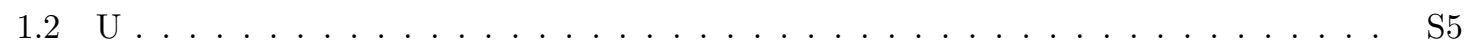

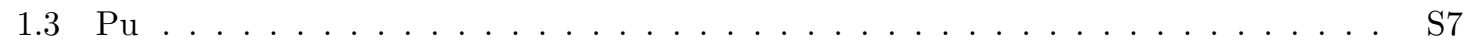

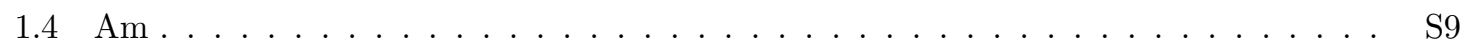

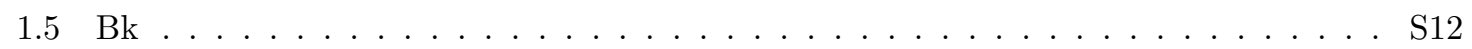

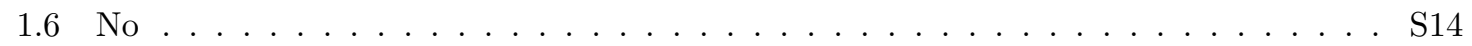

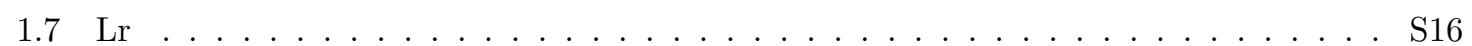

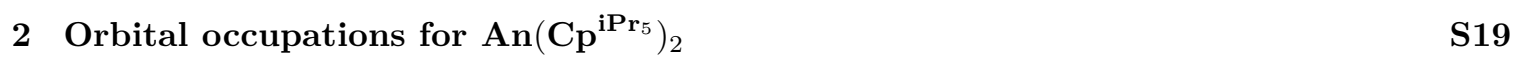

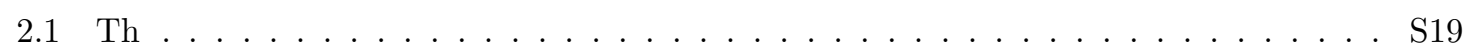

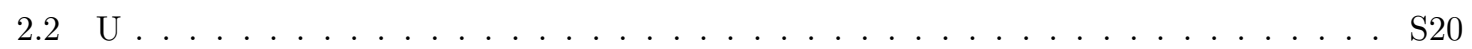

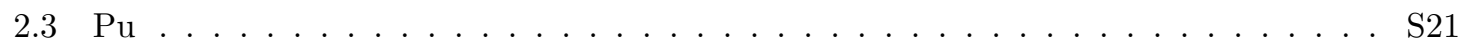

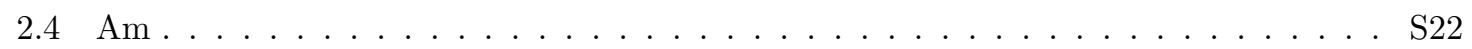

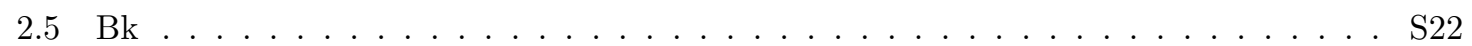

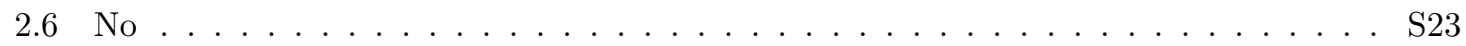

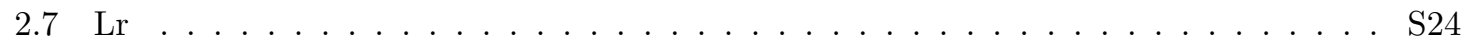

$\begin{array}{lll}3 & \text { Orbital contour plots and energies for } \mathbf{A n}\left(\mathbf{C p}^{\mathrm{iPr}}\right)_{2} & \mathbf{S 2 5}\end{array}$

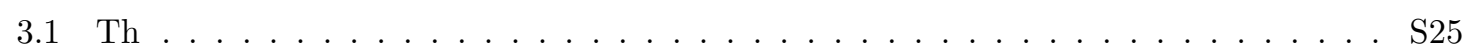

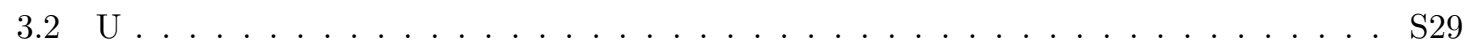

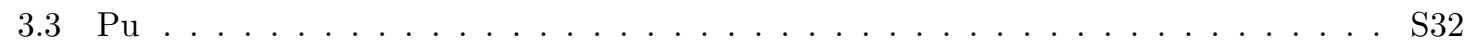

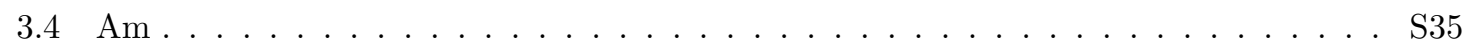

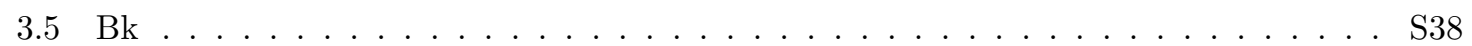

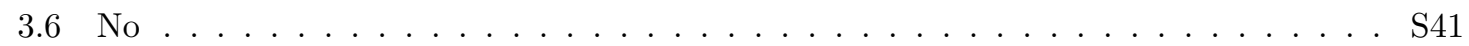

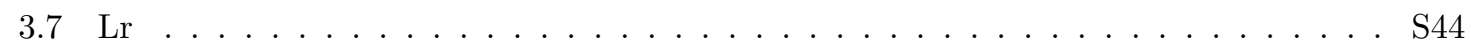

\begin{tabular}{|lll}
4 & Tabulated Absorption Spectra Data for $\operatorname{An}\left(\mathrm{Cp}^{\mathrm{iPr}{ }_{5}}\right)_{2}$ & $\mathbf{S} 45$
\end{tabular}

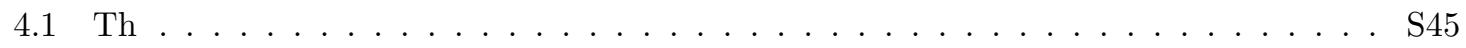

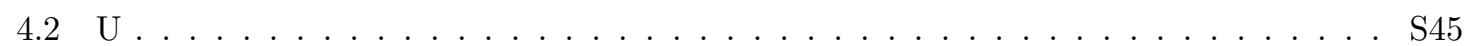

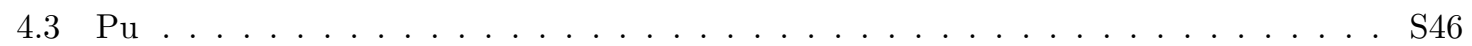

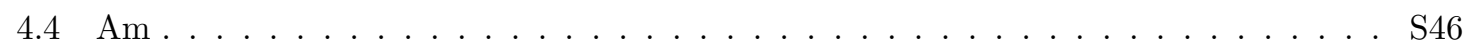

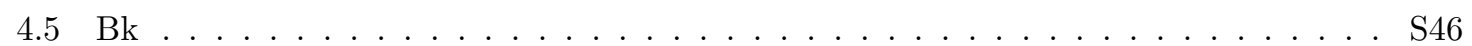

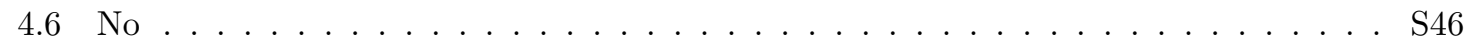

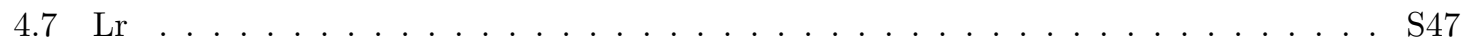


\begin{tabular}{|ll|l}
5 & Optimized coordinates for solvated $\left[\mathrm{An}\left(\mathrm{Cp}^{\mathrm{iPr}}\right)_{2}\right]^{+}$cations & $\mathrm{S} 47$
\end{tabular}

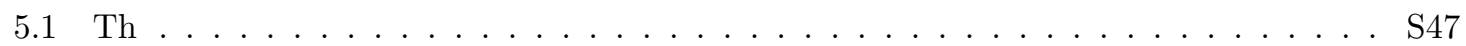

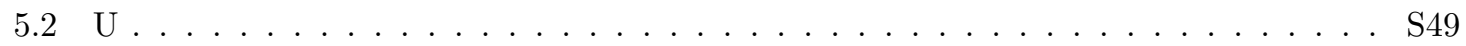

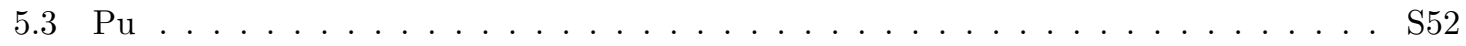

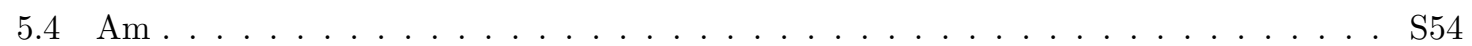

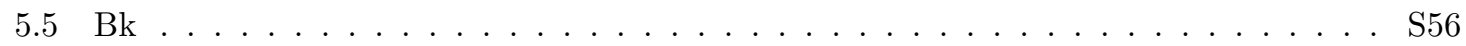

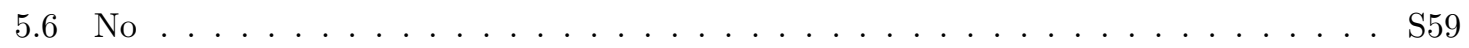

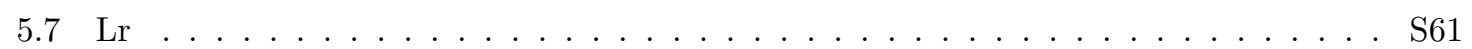

$\begin{array}{|ll|}6 & \text { Orbital occupations for solvated }\left[\mathrm{An}\left(\mathrm{Cp}^{\mathrm{iPr}}\right)_{2}\right]^{+} \text {cations }\end{array}$

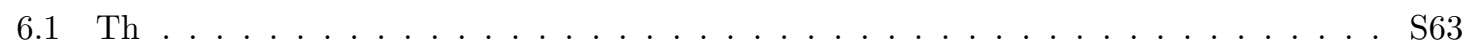

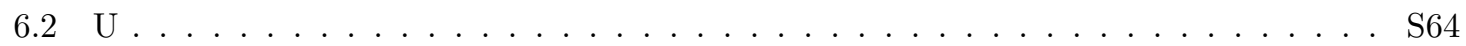

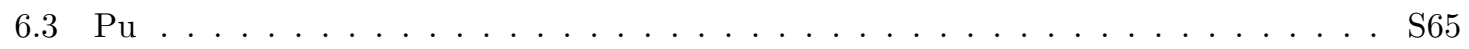

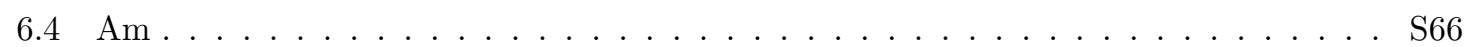

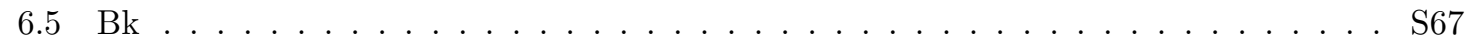

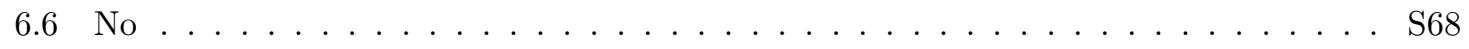

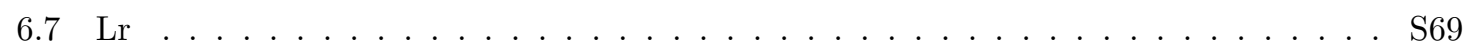

$\begin{array}{lll}7 & \text { Optimized coordinates for solvated }\left[\mathrm{Th}\left(\mathrm{Cp}^{\mathrm{iPr}}\right)_{2}\right]^{-} \text {anion } & \text { S70 }\end{array}$

8 Orbital occupations for solvated $\left[\mathrm{Th}\left(\mathrm{Cp}^{\mathrm{iPr_{5 }}}\right)_{2}\right]^{-}$anion $\quad$ S72

9 Natural Atomic Populations of HOMO for $\mathbf{A n}\left(\mathbf{C p}^{\left.\mathrm{iPr}_{5}\right)_{2}} \quad\right.$ S74 


\section{Optimized coordinates for $\mathrm{An}\left(\mathrm{Cp}^{\mathrm{iPr_{5 }}}\right)_{2}$}

\section{$1.1 \quad$ Th}

\$coord

\begin{tabular}{|c|c|}
\hline-0.26072625460968 & 2.29787937315277 \\
\hline-0.66455345806302 & 5.13945385617443 \\
\hline 0.14203722629259 & 5.98115569755650 \\
\hline 0.46874344891599 & 6.89068627613414 \\
\hline-2.26598199505128 & 0.46211837391211 \\
\hline-5.09326939232507 & 0.95615068664647 \\
\hline-5.64452518437875 & 1.98336418616955 \\
\hline-6.40858239294603 & 2.57514067379388 \\
\hline-1.13972763622731 & -2.01227451124927 \\
\hline-2.48326014025342 & -4.54852023346039 \\
\hline-3.63054564059340 & -4.75536921843447 \\
\hline-4.42946518746076 & -5.29916181391725 \\
\hline 1.56159157794525 & -1.70577241655922 \\
\hline 3.55853022274064 & -3.76729078944155 \\
\hline 3.40072458078426 & -4.92234399221709 \\
\hline 3.67102235511083 & -5.85020278668027 \\
\hline 2.10484430794304 & 0.95804918074360 \\
\hline 4.68255276790083 & 2.22020648008105 \\
\hline 5.73230901789532 & 1.71319332692546 \\
\hline 6.69828177637993 & 1.68353765066946 \\
\hline-2.72387744248884 & 5.43540472019717 \\
\hline-0.62813861459531 & 4.68884096733462 \\
\hline-0.56580204419018 & 7.91324926382119 \\
\hline 2.221258 & 6.01576659910378 \\
\hline-0.09779124534176 & 8.87630904588068 \\
\hline 2.55828859816380 & 6.82018562698833 \\
\hline-0.21482099826800 & 6.34480833428513 \\
\hline-6.01110149816858 & -0.91092896142163 \\
\hline-4.65345826258837 & 0.85153622028041 \\
\hline-7.70078972453182 & 1.90721878218561 \\
\hline-5.03492753318621 & 3. 97151607171484 \\
\hline-8.47209071544612 & 2.64992534138230 \\
\hline-5.69582732954154 & 4.54063030537839 \\
\hline-6.10065465017908 & 1.75634669110610 \\
\hline-0.99118759320476 & -5.99838977969244 \\
\hline-2.24785675691335 & -4.16256264054973 \\
\hline-4.19354774578645 & -6.73452323244829 \\
\hline-5.33301441277622 & -3.56123467991752 \\
\hline-5.13824877257633 & -7.23856511725668 \\
\hline-6.07850348187101 & -4.01392176791665 \\
\hline-3.55559092916775 & -5.25932638315312 \\
\hline 5.39851387634085 & -2.79627980019822 \\
\hline 3.26420638497480 & -3.42414141244068 \\
\hline 5.10903468419027 & -6.06938303786448 \\
\hline 1.73894336359751 & -6.17248014581872 \\
\hline
\end{tabular}

$4.78823732281176 \quad \mathrm{C}$ $5.18060259545873 \quad \mathrm{c}$ $7.86347945111519 \quad \mathrm{c}$ $3.14227748997980 \quad \mathrm{c}$ $4.78823732281176 \quad \mathrm{c}$ $5.18060259545873 \quad \mathrm{c}$ $7.86347945111519 \quad \mathrm{c}$ $3.14227748997980 \quad \mathrm{c}$ $4.78823732281176 \quad \mathrm{c}$ $5.18060259545873 \quad \mathrm{c}$ $7.86347945111519 \quad \mathrm{c}$ $3.14227748997980 \quad \mathrm{c}$ $4.78823732281176 \quad \mathrm{c}$ $5.18060259545873 \quad \mathrm{c}$ $7.86347945111519 \quad \mathrm{c}$ $3.14227748997980 \quad \mathrm{c}$ $4.78823732281176 \quad \mathrm{c}$ $5.18060259545873 \quad \mathrm{c}$ $7.86347945111519 \quad \mathrm{c}$ $3.14227748997980 \quad \mathrm{c}$ $5.07684341848665 \mathrm{~h}$ $9.31600356219072 \mathrm{~h}$ $8.25959441565163 \mathrm{~h}$ $8.07755672674043 \mathrm{~h}$ $3.50493639958401 \mathrm{~h}$ $3.09873755598148 \mathrm{~h}$ $1.24073288735571 \mathrm{~h}$ $5.07684341848665 \mathrm{~h}$ $9.31600356219072 \mathrm{~h}$ $8.25959441565163 \mathrm{~h}$ $8.07755672674043 \mathrm{~h}$ $3.50493639958401 \mathrm{~h}$ $3.09873755598148 \mathrm{~h}$ $1.24073288735571 \mathrm{~h}$ $5.07684341848665 \mathrm{~h}$ $9.31600356219072 \mathrm{~h}$ $8.25959441565163 \mathrm{~h}$ $8.07755672674043 \mathrm{~h}$ $3.50493639958401 \mathrm{~h}$ $3.09873755598148 \mathrm{~h}$ $1.24073288735571 \mathrm{~h}$ $5.07684341848665 \mathrm{~h}$ $9.31600356219072 \mathrm{~h}$ $8.25959441565163 \mathrm{~h}$ $8.07755672674043 \mathrm{~h}$ 


\begin{tabular}{|c|c|c|}
\hline 5.29647833134150 & -7.12360461362632 & 3.50493639958401 \\
\hline 3.90317860586259 & -5.00678915382380 & 1.24073288735571 \\
\hline 1.93910557701067 & -7.02137038613397 & 3.09873755598148 \\
\hline 4.32765265752132 & 4.27019382111510 & 5.07684341848665 \\
\hline 7.35110483031807 & 2.98343822430597 & 8.25959441565163 \\
\hline 4.26524724912222 & 2.04632686537533 & 9.31600356219072 \\
\hline 6.40774051599049 & -0.25356784508235 & 8.07755672674043 \\
\hline 8.41165240202274 & 2.83593534362012 & 3.50493639958401 \\
\hline 5.96788797175228 & 2.16496051158574 & 1.24073288735571 \\
\hline 7.27693663623808 & -0.32552377831614 & 3.09873755598148 \\
\hline 0.00000000000000 & -0.00000000000000 & 0.00000000000000 \\
\hline 0.26072625460968 & -2.29787937315277 & -4.78823732281176 \\
\hline 0.66455345806302 & -5.13945385617443 & -5.18060259545873 \\
\hline-0.14203722629259 & -5.98115569755650 & -7.86347945111519 \\
\hline-0.46874344891599 & -6.89068627613414 & -3.14227748997980 \\
\hline 2.26598199505128 & -0.46211837391211 & -4.78823732281176 \\
\hline 5.09326939232507 & -0.95615068664647 & -5.18060259545873 \\
\hline 5.64452518437875 & -1.98336418616955 & -7.86347945111519 \\
\hline 6.40858239294603 & -2.57514067379388 & -3.14227748997980 \\
\hline 1.13972763622731 & 2.01227451124927 & -4.78823732281176 \\
\hline 2.48326014025342 & 4.54852023346039 & -5.18060259545873 \\
\hline 3.63054564059340 & 4.75536921843447 & -7.86347945111519 \\
\hline 4.42946518746076 & 5.29916181391725 & -3.14227748997980 \\
\hline-1.56159157794525 & 1.70577241655922 & -4.78823732281176 \\
\hline-3.55853022274064 & 3.76729078944155 & -5.18060259545873 \\
\hline-3.40072458078426 & 4.92234399221709 & -7.86347945111519 \\
\hline-3.67102235511083 & 5.85020278668027 & -3.14227748997980 \\
\hline-2.10484430794304 & -0.95804918074360 & -4.78823732281176 \\
\hline-4.68255276790083 & -2.22020648008105 & -5.18060259545873 \\
\hline-5.73230901789532 & -1.71319332692546 & -7.86347945111519 \\
\hline-6.69828177637993 & -1.68353765066946 & -3.14227748997980 \\
\hline 2.72387744248884 & -5.43540472019717 & -5.07684341848665 \\
\hline 0.62813861459531 & -4.68884096733462 & -9.31600356219072 \\
\hline 0.56580204419018 & -7.91324926382119 & -8.25959441565163 \\
\hline-2.22125806637443 & -6.01576659910378 & -8.07755672674043 \\
\hline 0.09779124534176 & -8.87630904588068 & -3.50493639958401 \\
\hline-2.55828859816380 & -6.82018562698833 & -3.09873755598148 \\
\hline 0.21482099826800 & -6.34480833428513 & -1.24073288735571 \\
\hline 6.01110149816858 & 0.91092896142163 & -5.07684341848665 \\
\hline 4.65345826258837 & -0.85153622028041 & -9.31600356219072 \\
\hline 7.70078972453182 & -1.90721878218561 & -8.25959441565163 \\
\hline 5.03492753318621 & -3.97151607171484 & -8.07755672674043 \\
\hline 8.47209071544612 & -2.64992534138230 & -3.50493639958401 \\
\hline 5.69582732954154 & -4.54063030537839 & -3.09873755598148 \\
\hline 6.10065465017908 & -1.75634669110610 & -1.24073288735571 \\
\hline 0.99118759320476 & 5.99838977969244 & -5.07684341848665 \\
\hline 2.24785675691335 & 4.16256264054973 & -9.31600356219072 \\
\hline 4.19354774578645 & 6.73452323244829 & -8.25959441565163 \\
\hline 5.33301441277622 & 3.56123467991752 & -8.07755672674043 \\
\hline 5.13824877257633 & 7.23856511725668 & -3.50493639958401 \\
\hline 6.07850348187101 & 4.01392176791665 & -3.09873755598148 \\
\hline
\end{tabular}


3.55559092916775
-5.39851387634085
-3.26420638497480
-5.10903468419027
-1.73894336359751
-5.29647833134150
-3.90317860586259
-1.93910557701067
-4.32765265752132
-7.35110483031807
-4.26524724912222
-6.40774051599049
-8.41165240202274
-5.96788797175228
-7.27693663623808 \$end

\section{$1.2 \mathrm{U}$}

\$coord

$-0.26513831890914$

$-0.66935561012161$

0.13574833864093

0.47126725704028

$-2.26803450539514$

$-5.09397645802300$

$-5.66965844870587$

$-6.39111025106686$

$-1.13658409308259$

$-2.47889497882844$

$-3.63978996454409$

$-4.42119061804747$

1.56558690479762

3. 56193510656560

3. 42014453870684

3. 65866417837135

2. 10417001258927

4.68029194040745

5.75355553590227

6.68236943370268

$-2.72800359962922$

$-0.65585688799653$

$-0.54890677660271$

2.21443391033264

$-0.11690020583459$

2.56166232623525

$-0.18480790303496$

$-6.01811071469402$

$-4.70919158818349$

$-7.73163757798677$
5.25932638315312

2. 79627980019822

3. 42414141244068

6.06938303786448

6. 17248014581872

7.12360461362632

5.00678915382380

7.02137038613397

$-4.27019382111510$

$-2.98343822430597$

$-2.04632686537533$

0.25356784508235

$-2.83593534362012$

$-2.16496051158574$

0.32552377831614

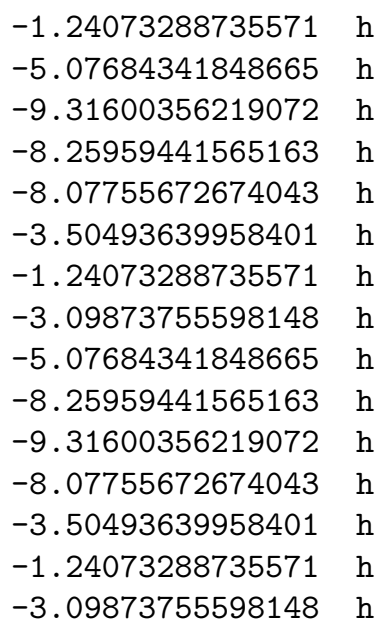

2.29860394365224 5.13863699525779

6.00553899210288

6.87313501394080

0.45814615600770

0.95133114473355

1. 98491795098179

2.57211731966589

$-2.01545404742433$

$-4.55068201325610$

$-4.77879223351628$

$-5.28347908733499$

$-1.70376526007950$

$-3.76380730091866$

$-4.93837397646893$

$-5.83748697448820$

0.96246920784393

2. 22452117418335

1. 72670926690052

1. 67571372821647

5.44143397687055

4. 73843624085546

7.95117422164024

6.01521072080029

8.86014285652141

6.82755143757396

6.30399317318037

$-0.91299002728176$

0.84050035810033

1.93500659299623
$4.69828886702405 \quad \mathrm{c}$ $5.08738208734420 \quad \mathrm{c}$ $7.76220069452461 \quad \mathrm{c}$ $3.03830499901027 \quad \mathrm{c}$ $4.69828886702405 \quad \mathrm{c}$ $5.08738208734420 \quad \mathrm{c}$ $7.76220069452461 \quad \mathrm{c}$ $3.03830499901027 \quad \mathrm{c}$ $4.69828886702405 \quad \mathrm{c}$ $5.08738208734420 \quad c$ $7.76220069452461 \quad \mathrm{C}$ $3.03830499901027 \quad \mathrm{c}$ $4.69828886702405 \quad \mathrm{c}$ $5.08738208734420 \quad \mathrm{c}$ $7.76220069452461 \quad \mathrm{C}$ $3.03830499901027 \quad \mathrm{c}$ 4.69828886702405 c $5.08738208734420 \quad \mathrm{c}$ $7.76220069452461 \quad \mathrm{c}$ $3.03830499901027 \quad \mathrm{c}$ $4.98071493470536 \mathrm{~h}$ $9.22494304729354 \mathrm{~h}$ $8.13497707196052 \mathrm{~h}$ $7.98492474140065 \mathrm{~h}$ $3.35530641690311 \mathrm{~h}$ $3.02631269050297 \mathrm{~h}$ $1.13511901557340 \mathrm{~h}$ $4.98071493470536 \mathrm{~h}$ $9.22494304729354 \mathrm{~h}$ $8.13497707196052 \mathrm{~h}$ 


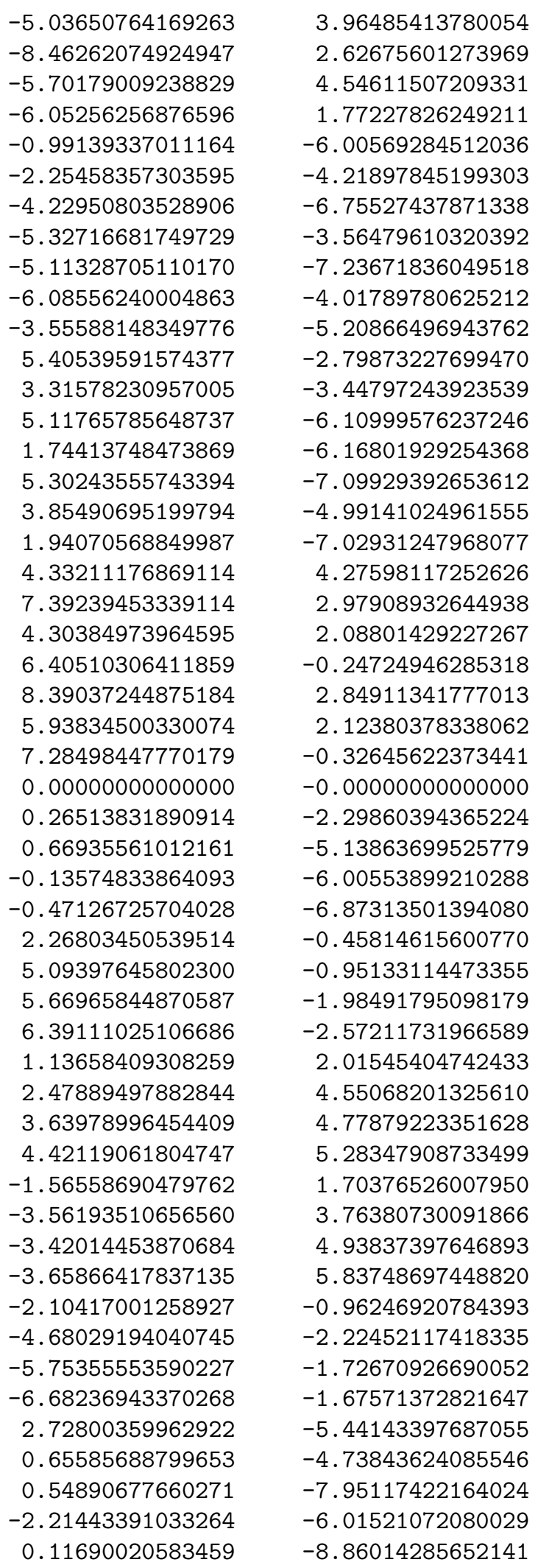

3.96485413780054

2. 62675601273969

4.54611507209331

1. 77227826249211

$-6.00569284512036$

$-4.21897845199303$

$-6.75527437871338$

$-3.56479610320392$

$-7.23671836049518$

$-4.01789780625212$

$-5.20866496943762$

$-2.79873227699470$

$-3.44797243923539$

$-6.10999576237246$

$-6.16801929254368$

$-7.09929392653612$

$-4.99141024961555$

$-7.02931247968077$

4. 27598117252626

2. 97908932644938

2.08801429227267

$-0.24724946285318$

2. 84911341777013

2. 12380378338062

$-0.32645622373441$

$-0.00000000000000$

$-2.29860394365224$

$-5.13863699525779$

$-6.00553899210288$

$-6.87313501394080$

$-0.45814615600770$

$-0.95133114473355$

$-1.98491795098179$

$-2.57211731966589$

2.01545404742433

4. 55068201325610

4. 77879223351628

5. 28347908733499

1. 70376526007950

3. 76380730091866

4.93837397646893

5.83748697448820

$-0.96246920784393$

$-2.22452117418335$

$-1.72670926690052$

$-1.67571372821647$

$-5.44143397687055$

$-4.73843624085546$

$-7.95117422164024$

$-6.01521072080029$

$-8.86014285652141$

$7.98492474140065 \mathrm{~h}$ $3.35530641690311 \mathrm{~h}$ $3.02631269050297 \mathrm{~h}$ $1.13511901557340 \mathrm{~h}$ $4.98071493470536 \mathrm{~h}$ $9.22494304729354 \mathrm{~h}$ $8.13497707196052 \mathrm{~h}$ $7.98492474140065 \mathrm{~h}$ $3.35530641690311 \mathrm{~h}$ $3.02631269050297 \mathrm{~h}$ $1.13511901557340 \mathrm{~h}$ $4.98071493470536 \mathrm{~h}$ $9.22494304729354 \mathrm{~h}$ $8.13497707196052 \mathrm{~h}$ $7.98492474140065 \mathrm{~h}$ $3.35530641690311 \mathrm{~h}$ $1.13511901557340 \mathrm{~h}$ $3.02631269050297 \mathrm{~h}$ $4.98071493470536 \mathrm{~h}$ $8.13497707196052 \mathrm{~h}$ $9.22494304729354 \mathrm{~h}$ $7.98492474140065 \mathrm{~h}$ $3.35530641690311 \mathrm{~h}$ $1.13511901557340 \mathrm{~h}$ $3.02631269050297 \mathrm{~h}$ $0.00000000000000 \mathrm{u}$ $-4.69828886702405 \mathrm{c}$ $-5.08738208734420 \quad \mathrm{c}$ $-7.76220069452461 \quad \mathrm{c}$ $-3.03830499901027 \quad \mathrm{c}$ $-4.69828886702405 \quad \mathrm{c}$ $-5.08738208734420 \quad c$ $-7.76220069452461 \quad \mathrm{c}$ $-3.03830499901027 \quad c$ $-4.69828886702405 \quad \mathrm{c}$ $-5.08738208734420 \quad c$ $-7.76220069452461 \quad \mathrm{c}$ $-3.03830499901027 \quad c$ $-4.69828886702405 \quad \mathrm{c}$ $-5.08738208734420 \quad c$ $-7.76220069452461 \quad \mathrm{c}$ $-3.03830499901027 \quad c$ $-4.69828886702405 \quad \mathrm{c}$ $-5.08738208734420 \quad \mathrm{c}$ $-7.76220069452461 \quad \mathrm{c}$ $-3.03830499901027 \quad c$ $-4.98071493470536 \mathrm{~h}$ $-9.22494304729354 \mathrm{~h}$ $-8.13497707196052 \mathrm{~h}$ $-7.98492474140065 \mathrm{~h}$ $-3.35530641690311 \mathrm{~h}$ 


$\begin{array}{rr}-2.56166232623525 & -6.82755143757396 \\ 0.18480790303496 & -6.30399317318037 \\ 6.01811071469402 & 0.91299002728176 \\ 4.70919158818349 & -0.84050035810033 \\ 7.73163757798677 & -1.93500659299623 \\ 5.03650764169263 & -3.96485413780054 \\ 8.46262074924947 & -2.62675601273969 \\ 5.70179009238829 & -4.54611507209331 \\ 6.05256256876596 & -1.77227826249211 \\ 0.99139337011164 & 6.00569284512036 \\ 2.25458357303595 & 4.21897845199303 \\ 4.22950803528906 & 6.75527437871338 \\ 5.32716681749729 & 3.56479610320392 \\ 5.11328705110170 & 7.23671836049518 \\ 6.08556240004863 & 4.01789780625212 \\ 3.55588148349776 & 5.20866496943762 \\ -5.40539591574377 & 2.79873227699470 \\ -3.31578230957005 & 3.44797243923539 \\ -5.11765785648737 & 6.10999576237246 \\ -1.74413748473869 & 6.16801929254368 \\ -5.30243555743394 & 7.09929392653612 \\ -3.85490695199794 & 4.99141024961555 \\ -1.94070568849987 & 7.02931247968077 \\ -4.33211176869114 & -4.27598117252626 \\ -7.39239453339114 & -2.97908932644938 \\ -4.30384973964595 & -2.08801429227267 \\ -6.40510306411859 & 0.24724946285318 \\ -8.39037244875184 & -2.84911341777013 \\ -5.93834500330074 & -2.12380378338062 \\ -7.28498447770179 & 0.32645622373441\end{array}$
\$end

\section{3 $\mathrm{Pu}$}

\$coord
$-0.25006522998221$
$-0.66534494752230$
0.03469702751187
0.56927072158870
$-2.24917322546076$
$-5.08340607055342$
$-5.72533360726335$
$-6.34992782949231$
$-1.11362824045353$
$-2.45626633616560$
$-3.77659168151473$
$-4.25442461399140$
1.58083773609100
3. 56769536006977
3.40767494921508

\footnotetext{
2. 30193351844339

5. 14672024740571

6.05105405907278

6.85920855142456

0.45465282884749

0.94106744137032

2. 03014359953659

2. 51328993934404

$-2.00837955786677$

$-4.53864248885049$

$-4.72198125547989$

$-5.33194957305507$

$-1.69299313548283$

$-3.75224801671578$

$-4.95167359223600$
}

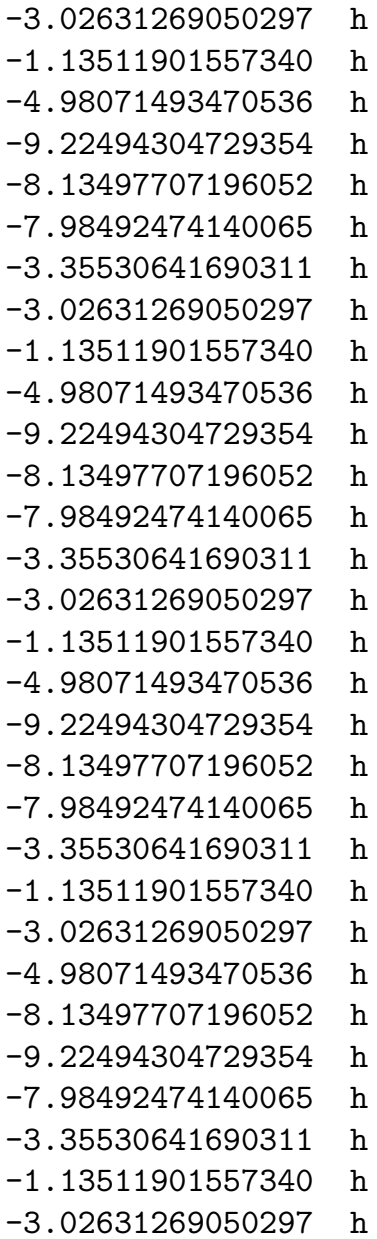

\author{
$h$
} $\mathrm{h}$ (n) h $\mathrm{h}$ $\mathrm{h}$ (n)

(n)

(n)

h

h

h

h

h

h

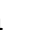

h

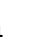

h

h




\begin{tabular}{|c|c|c|}
\hline 3.66152445735011 & -5.79095631280223 & 3.17102531044067 \\
\hline 2.11861987401523 & 0.96355487363243 & 4.64890973740251 \\
\hline 4.69794511071057 & 2.22760697812738 & 4.98032022600603 \\
\hline 5.76967845440413 & 1.86213143595031 & 7.67667083205622 \\
\hline 6.68779402407401 & 1.53751637383626 & 2.96114449731275 \\
\hline-2.71749627380707 & 5.44715661959445 & 4.62777944345668 \\
\hline-0.81330539236904 & 4.81067881549831 & 8.97710432993680 \\
\hline-0.65071094094746 & 8.00626784269362 & 7.84264179998701 \\
\hline 2.10510933705816 & 6.05351998254636 & 7.81813914711928 \\
\hline-0.14044326687576 & 8.82341433493492 & 2.99888538204098 \\
\hline 2.65119496855163 & 6.92849863458797 & 3.00309169540497 \\
\hline 0.12133765420583 & 6.17317001170369 & 0.90070637940759 \\
\hline-5.99489053868453 & -0.93158616423270 & 4.84018039784306 \\
\hline-4.79912370127267 & 0.92206880116190 & 9.07535899380706 \\
\hline-7.79596339940243 & 1.98851056048507 & 7.88862037587999 \\
\hline-5.09635096046942 & 4.01511415868501 & 7.75057426722010 \\
\hline-8.43645161890786 & 2.43118181683818 & 3.00501200293725 \\
\hline-5.79277897911065 & 4.52644657535745 & 2.89029899335752 \\
\hline-5.83496367924541 & 1.78013987961292 & 0.93576625053825 \\
\hline-0.95863655274396 & -5.98690780295879 & 5.23962619527587 \\
\hline-2.46830052422929 & -4.15287410472778 & 9.34526995578397 \\
\hline-4.40211383139835 & -6.68920196486622 & 8.18226843395280 \\
\hline-5.46223833750581 & -3.49115824034827 & 7.92892344620538 \\
\hline-4.95565461554906 & -7.28427105014619 & 3. 35692019798091 \\
\hline-5.92031044831000 & -4.07902336175794 & 2.90779681331980 \\
\hline-3.26865321636065 & -5.30815651821757 & 1. 20997062641254 \\
\hline 5.41852568296918 & -2.79998516564911 & 5.18010257668175 \\
\hline 3.35975038963667 & -3.46548522720254 & 9.39038234818023 \\
\hline 5.07285201014383 & -6.17523985269260 & 8.27316807317078 \\
\hline 1.69670031589123 & -6.13005022789864 & 8.15329897788573 \\
\hline 5.25477845596984 & -7.11467909940461 & 3.49100455628654 \\
\hline 3.95557068621969 & -4.92052154724788 & 1.28819112513121 \\
\hline 1.90679615002811 & -6.92434212537751 & 3.07852231630614 \\
\hline 4.37156723798356 & 4.27296172203133 & 4.75813270945982 \\
\hline 7.43995345403610 & 3.09324890625521 & 7.97464383319480 \\
\hline 4.33320874756365 & 2.34156886385240 & 9.11776563121454 \\
\hline 6.37203493549962 & -0.11162321033020 & 8.01476793418412 \\
\hline 8.39968801651632 & 2.72816385750676 & 3.17164718682901 \\
\hline 5.93031998977604 & 1.85903077750033 & 1.03944586019876 \\
\hline 7.29317443064860 & -0.46041225601464 & 3.08452039953786 \\
\hline 0.11193605515859 & -0.42047242983558 & 0.00411889041685 \\
\hline 0.26499577085904 & -2.29078829009918 & -4.85479159787981 \\
\hline 0.66025219794455 & -5.12294959321880 & -5.28958996983144 \\
\hline-0.26357224306719 & -6.00836709581336 & -7.91746870822384 \\
\hline-0.36822774794291 & -6.83761422906254 & -3.16284895951459 \\
\hline 2.26305425789496 & -0.45605588655951 & -4.76701048802731 \\
\hline 5.08702768652875 & -0.95645661685579 & -5.13103093199812 \\
\hline 5.71559626716335 & -1.94673442271765 & -7.80878146304597 \\
\hline 6.30372063140413 & -2.62484291121056 & -3.06750827122792 \\
\hline 1.13812525955674 & 2.01339659309204 & -4.57597705018366 \\
\hline 2.48294935963769 & 4.55517965459291 & -4.87302157895033 \\
\hline
\end{tabular}




\begin{tabular}{|c|c|c|}
\hline 3.57806868128247 & 4.90500859449249 & -7.56270245867706 \\
\hline 4.49149699325090 & 5.16186038124610 & -2.84789803813555 \\
\hline-1.56356098920064 & 1.70487850479735 & -4.53971303342731 \\
\hline-3.57031871994536 & 3.76561922208424 & -4.85109071789661 \\
\hline-3.50027446038618 & 4.95537352516477 & -7.52303590569181 \\
\hline-3.61473023044447 & 5.84153747159075 & -2.80525773692738 \\
\hline-2.09982644428583 & -0.96136672105480 & -4.70588720486054 \\
\hline-4.67637912645971 & -2.22522541104297 & -5.07392174875667 \\
\hline-5.84424379268610 & -1.64221163429624 & -7.69025950509557 \\
\hline-6.61706044383895 & -1.77465256276118 & -2.94449182712729 \\
\hline 2.72130426426550 & -5.42616091512881 & -5.28722496917247 \\
\hline 0.49898051009699 & -4.76601705442126 & -9.41678340721369 \\
\hline 0.37659913190576 & -7.96833437221605 & -8.29609127039137 \\
\hline-2.34817446082485 & -5.98377854515130 & -8.07007447564894 \\
\hline 0.15042332490696 & -8.83984294602710 & -3.50193650997581 \\
\hline-2.45043198464149 & -6.74411322034416 & -2.99757250848959 \\
\hline 0.44458197609856 & -6.29785202873212 & -1.30720619771223 \\
\hline 6.03139348103182 & 0.89316058824086 & -4.97070379542267 \\
\hline 4.84067232821064 & -0.72899623017983 & -9.26575498797912 \\
\hline 7.78904450604811 & -1.95924222658584 & -8.11733472926256 \\
\hline 5.02001441670095 & -3.89523898438238 & -8.11045831309151 \\
\hline 8.380332 & -2.73657573669733 & -3.32764270916199 \\
\hline 5.56104061090028 & -4.57956609619048 & -3.07480930518065 \\
\hline 5.96659656964019 & -1.82036021266634 & -1.16440144862903 \\
\hline 1.01093872130427 & 6.01203843948456 & -4.64753155107528 \\
\hline 2.14814582610504 & 4.44755391781017 & -9.01754045539093 \\
\hline 4.19996423277478 & 6.88630490243700 & -7.84867909288673 \\
\hline 5.23412869274508 & 3.67058731504408 & -7.89039557301321 \\
\hline 5.116093 & 7.15637202523913 & -3.00512582823239 \\
\hline 6.19151741200291 & 3.95691946578569 & -3.02509409804868 \\
\hline 3.70725305971537 & 4.88257070581329 & -0.93229553262067 \\
\hline-5.40837723503246 & 2.79509316100727 & -4.69436921970147 \\
\hline-3.43536292489624 & 3.47916115575544 & -9.00200499010134 \\
\hline-5.20127063844003 & 6.13769641435380 & -7.84452998751923 \\
\hline-1.82420474674112 & 6.18077491070286 & -7.77160479828476 \\
\hline-5.36024225062245 & 6.99032948041094 & -2.97063605305618 \\
\hline-3.56302341203644 & 4.99598196038605 & -0.89775951924656 \\
\hline-1.98992144747439 & 7.14763380883561 & -2.96227335642172 \\
\hline-4.31200153961509 & -4.27732178718165 & -5.05799520930023 \\
\hline-7.49505776874964 & -2.88395926182389 & -8.04800824411590 \\
\hline-4.44463082636737 & -1.95307996492461 & -9.21199704590459 \\
\hline-6.50214370296155 & 0.33748271160619 & -7.82255497015663 \\
\hline-8.29467478476979 & -3.00327266469465 & -3.20768656909093 \\
\hline-5.79567675145435 & -2.22001743785167 & -1.07275945471066 \\
\hline-7.28861116635951 & 0.20327242347924 & -2.88352983536845 \\
\hline
\end{tabular}
\$end

\section{$1.4 \mathrm{Am}$}

\$coord 


\begin{tabular}{|c|c|c|}
\hline 0.08974843508045 & 2.20377124725922 & 4.94344786702553 \\
\hline 0.12627186595770 & 5.07117394300717 & 5.36908612992581 \\
\hline 1.14285342729087 & 5.85093122408064 & 8.00007394269914 \\
\hline 1.42330119941171 & 6.61261269351202 & 3.25200541881013 \\
\hline-2.17615506137724 & 0.69707666041423 & 4.85133667746366 \\
\hline-4.89802591099547 & 1.61446593273564 & 5.23835724720010 \\
\hline-5.37607605719023 & 2.71120145970641 & 7.90969291113979 \\
\hline-5.90629479180805 & 3.42673665714729 & 3.17952096213209 \\
\hline-1.44448322631804 & -1.92186060071118 & 4.67826234116126 \\
\hline-3.15426540737804 & -4.23576410343658 & 5.04206392242982 \\
\hline-4.29012828653118 & -4.37674824851557 & 7.73850877054067 \\
\hline-5.24567030650058 & -4.62905468232084 & 3.04285827462460 \\
\hline 1.28491088988205 & -2.03290080752135 & 4.66753318226836 \\
\hline 2.95236040708153 & -4.37757858506028 & 5.03815275559173 \\
\hline 2.65966817955444 & -5.55382579804868 & 7.70532692895888 \\
\hline 2.76139388829872 & -6.45508640084121 & 2.99760198338242 \\
\hline 2.22845527802684 & 0.52011697459644 & 4.83293272130648 \\
\hline 4.96951670157474 & 1.37360885531527 & 5.24574885475701 \\
\hline 6.02057042257740 & 0.62704994388441 & 7.87457468431998 \\
\hline 6.86186512752298 & 0.64587390614229 & 3.14413003597912 \\
\hline-1.86730945366256 & 5.68068565891602 & 5.34320726493292 \\
\hline 0.17508567760938 & 4.77946861063627 & 9.51241691895129 \\
\hline 0.81103637060541 & 7.89462255393654 & 8.33184396212657 \\
\hline 3. 19619874036310 & 5.50781736842424 & 8.19152904160903 \\
\hline 1.18911737448938 & 8.67027212704770 & 3.57885349882876 \\
\hline 3.47538004957583 & 6.23077859903994 & 3.11932642783351 \\
\hline 0.56999599308270 & 6.20023191798994 & 1.38007611458296 \\
\hline-6.09982007621844 & -0.08339630274693 & 5.10610093430928 \\
\hline-4.69613954635925 & 1. 39553214714758 & 9.38530887940771 \\
\hline-7.42709177031582 & 3.02682166060082 & 8.21028642128555 \\
\hline-4.41005441448853 & 4.54310946015359 & 8.19834993620946 \\
\hline-7.94228421324958 & 3.82802865265902 & 3.47913733751506 \\
\hline-4.90695758498741 & 5.26498078788150 & 3.16397616484439 \\
\hline-5.72585540971341 & 2.57672780395016 & 1.27328369007776 \\
\hline-1.90804031126349 & -5.89770335569515 & 4.86328611098466 \\
\hline-2.81291928154328 & -4.11086093108583 & 9.19349876631883 \\
\hline-5.18914735297181 & -6.24477675805508 & 8.05270768581536 \\
\hline-5.75583841374319 & -2.91691841239728 & 8.04624719076598 \\
\hline-6.20495587634411 & -6.46846593142576 & 3.34794331592936 \\
\hline-6.71754308120276 & -3.14439759514312 & 3.11685304662071 \\
\hline-4.44509681132213 & -4.64519506561876 & 1.11273262452895 \\
\hline 4.91961381231875 & -3.69261581957376 & 4.93749531754681 \\
\hline 2.79133473478730 & -4.09207146410266 & 9.19412680056092 \\
\hline 4.16823523483058 & -6.97027157050317 & 8.04419001751384 \\
\hline 0.82354867875953 & -6.52894026431870 & 7.92901843203252 \\
\hline 4.23055467572732 & -7.91690318667683 & 3.31619091285777 \\
\hline 3.05235607154373 & -5.66339777258980 & 1.08607023358117 \\
\hline 0.90900328952637 & -7.42501263290991 & 3.01294487385122 \\
\hline 4.92302723604517 & 3.45874095929719 & 5.23846789079488 \\
\hline 7.84487809956071 & 1.59775238406632 & 8.23056676692975 \\
\hline 4.68737160278319 & 1.16748127465011 & 9.39183854121839 \\
\hline
\end{tabular}




\begin{tabular}{|c|c|c|}
\hline 6.36240399260683 & -1.42963095002282 & 8.02818715578321 \\
\hline 8.72429643193219 & 1.54281266055979 & 3.49464962040943 \\
\hline 6.20221838199735 & 1. 29762915424491 & 1. 26678844035194 \\
\hline 7.17538425595858 & -1.41772416121729 & 3.02629826454714 \\
\hline-0.00512540609607 & 0.40732494430196 & 0.00243275538391 \\
\hline-0.10352147106192 & -2.41703977207628 & -4.64163201980648 \\
\hline-0.12880897361979 & -5.29549260093303 & -4.99758169775796 \\
\hline-0.99839849743808 & -6.08340387611512 & -7.68217363327115 \\
\hline-1.55941379789226 & -6.84786901436182 & -2.98226481825922 \\
\hline 2.16819848483550 & -0.90606202800625 & -4.73615371835410 \\
\hline 4.89096030482062 & -1.82583329309574 & -5.13149396696721 \\
\hline 5.31606172920926 & -3.00400187304099 & -7.77909251873746 \\
\hline 5.98717647955843 & -3.56800133363489 & -3.06182160251783 \\
\hline 1.42825344872239 & 1.71151031762154 & -4.90350436402317 \\
\hline 3.14402368918744 & 4.01004133855565 & -5.32871601895334 \\
\hline 4.44314955055840 & 4.02194415380631 & -7.95150427181461 \\
\hline 5.08757102938532 & 4.52455402390053 & -3.20904634301414 \\
\hline-1.29266297236011 & 1.82095558525980 & -4.91473119458354 \\
\hline-2.94959259219348 & 4.16262363407807 & -5.33133858520685 \\
\hline-2.63434926043982 & 5.35436356840484 & -7.98553853136311 \\
\hline-2.75235391059462 & 6.20059856881934 & -3.24566119352018 \\
\hline-2.24223567686961 & -0.72659357330541 & -4.75454891670160 \\
\hline-4.98316355244718 & -1.59067010428263 & -5.13610291558899 \\
\hline-5.98459358874101 & -1.02563006505142 & -7.82932758084763 \\
\hline-6.89747591228530 & -0.68571075074506 & -3.12376251147624 \\
\hline 1.86046266659398 & -5.90207308182213 & -4.84520068387934 \\
\hline 0.00506646955937 & -4.98906308991995 & -9.15377239898860 \\
\hline-0.61156826449471 & -8.11907614156463 & -8.00301657252563 \\
\hline-3.05031827960400 & -5.78584872443364 & -7.95503424327764 \\
\hline-1.23460001519739 & -8.89738188154252 & -3.28376274597258 \\
\hline-3.62606308461227 & -6.53318403759789 & -3.04600064813132 \\
\hline-0.89757854815666 & -6.37303405122825 & -1.05873923838898 \\
\hline 6.07860199168856 & -0.11182489163787 & -5.08551256423945 \\
\hline 4.55051628561410 & -1.76924062891543 & -9.28256067609373 \\
\hline 7.36506127196867 & -3.27351563804483 & -8.13417220745717 \\
\hline 4.39702589586863 & -4.87330884720058 & -7.95841916237760 \\
\hline 8.02822329656384 & -3.90467147530402 & -3.40443143729822 \\
\hline 5.04964298751070 & -5.43585358020169 & -3.00749476756197 \\
\hline 5.79764905490461 & -2.70522389359727 & -1.16633104390539 \\
\hline 1.88446266264141 & 5.67186315906430 & -5.32777250618310 \\
\hline 3.04573337658305 & 3.69354224454164 & -9.47174305819956 \\
\hline 5.36382889137675 & 5.87364644642553 & -8.29875371951171 \\
\hline 5.91615530243450 & 2.54718406610034 & -8.11061128599247 \\
\hline 6.09245956281882 & 6.33269505552492 & -3.55031884716454 \\
\hline 6.53504883741126 & 3.02259680547229 & -3.06383917358955 \\
\hline 4.15046542315094 & 4.69219190981935 & -1.34081783318172 \\
\hline-4.92185108677729 & 3.49216882680266 & -5.25281603000826 \\
\hline-2.82211274025713 & 3.90008951271080 & -9.47584190535967 \\
\hline-4.10515856918830 & 6.81378278855766 & -8.30739840534200 \\
\hline-0.77249578964039 & 6.27462483992357 & -8.22675441088596 \\
\hline-4.15066988590479 & 7.72970393502495 & -3.56557666709160 \\
\hline
\end{tabular}




$$
\begin{aligned}
& -3.16492119320881 \\
& -0.86165561603203 \\
& -4.95610390347324 \\
& -7.81724591409496 \\
& -4.63819218617517 \\
& -6.29616280185752 \\
& -8.76947253398592 \\
& -6.27313057962498 \\
& -7.18284704341317
\end{aligned}
$$

\$end

\subsection{Bk}

$\$$ coord

$$
\begin{array}{rr}
-0.26953359201756 & 2.30262510281352 \\
-0.67520298111439 & 5.13956276304090 \\
0.13134758203272 & 6.03192888790232 \\
0.46975143734054 & 6.85587129265764 \\
-2.27321706910398 & 0.45520860939500 \\
-5.09666385251493 & 0.94605604242557 \\
-5.69611663965029 & 1.98888750901485 \\
-6.37515989049258 & 2.56534090620030 \\
-1.13539182049513 & -2.02129071023583 \\
-2.47470850897284 & -4.55486797355969 \\
-3.65174126922046 & -4.80272880753106 \\
-4.40981693338003 & -5.27040341989538 \\
1.57150633348937 & -1.70443496946519 \\
3.56720988172116 & -3.76111926435381 \\
3.43921641715135 & -4.95713715081729 \\
3.64974314149890 & -5.82262935411931 \\
2.10663614812730 & 0.96789196749248 \\
4.67936546088097 & 2.23036843244703 \\
5.77729390968666 & 1.73904956143115 \\
6.66548224503320 & 1.67182057515677 \\
-2.73326663803638 & 5.44690651295416 \\
-0.67553887997517 & 4.78559181572194 \\
-0.53496029647296 & 7.98808439075405 \\
2.20957764448027 & 6.02159477112727 \\
-0.11073587434090 & 8.84892132271622 \\
2.55931874620588 & 6.80535762893607 \\
-0.18614876224009 & 6.28164805260197 \\
-6.02494177410684 & -0.91630436760218 \\
-4.76012127494445 & 0.83635354538864 \\
-7.76243153546830 & 1.95967635332446 \\
-5.04407990303403 & 3.96220833456072 \\
-8.45004355321067 & 2.62915099572658 \\
-5.68140673211858 & 4.53702793099590 \\
-6.03172544451392 & 1.76409800760760 \\
-0.99035215860079 & -6.01321375617231 \\
-2.26637785851201 & -4.26869689806029
\end{array}
$$

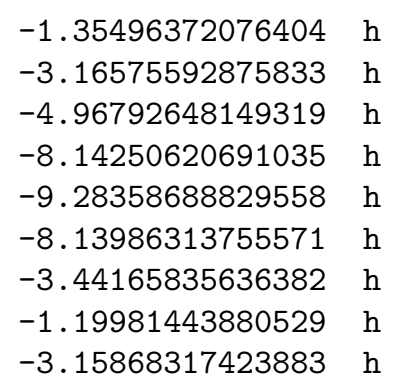

$\mathrm{h}$

1.01894624503810

$-1.57510954194905$

$-1.21883514488078$

1. 38676386820992
$4.63928549464807 \quad \mathrm{C}$

$5.02243822940706 \quad \mathrm{c}$

$7.68868271597103 \quad \mathrm{c}$

2.96038272598829 C

$4.63928549464807 \quad \mathrm{c}$

$5.02243822940706 \quad \mathrm{c}$

$7.68868271597103 \quad \mathrm{c}$

2.96038272598829 c

$4.63928549464807 \quad \mathrm{c}$

5.02243822940706 c

$7.68868271597103 \quad \mathrm{c}$

$2.96038272598829 \quad \mathrm{c}$

$4.63928549464807 \quad \mathrm{c}$

5.02243822940706 c

$7.68868271597103 \mathrm{C}$

2.96038272598829 C

$4.63928549464807 \quad \mathrm{C}$

$5.02243822940706 \quad \mathrm{c}$

$7.68868271597103 \quad \mathrm{C}$

$2.96038272598829 \quad \mathrm{c}$

$4.91414686797613 \mathrm{~h}$

$9.16036996777358 \mathrm{~h}$

$8.04140543887694 \mathrm{~h}$

$7.91589044850113 \mathrm{~h}$

$3.24897204489626 \mathrm{~h}$

$2.95499646136592 \mathrm{~h}$

$1.06051547261897 \mathrm{~h}$

$4.91414686797613 \mathrm{~h}$

$9.16036996777358 \mathrm{~h}$

$8.04140543887694 \mathrm{~h}$

$7.91589044850113 \mathrm{~h}$

$3.24897204489626 \mathrm{~h}$

$2.95499646136592 \mathrm{~h}$

$1.06051547261897 \mathrm{~h}$

$4.91414686797613 \mathrm{~h}$

$9.16036996777358 \mathrm{~h}$ 


\begin{tabular}{|c|c|}
\hline-4.26248622779044 & -6.77693779745011 \\
\hline-5.32699046652556 & -3.57281534986062 \\
\hline-5.11167824796019 & -7.22401664580158 \\
\hline-6.07062121056762 & -4.00132015967298 \\
\hline-3.54166257327707 & -5.19137552441452 \\
\hline 5.41287047925977 & -2.80006611533069 \\
\hline 3.35942272703385 & -3.47455331606116 \\
\hline 5.12807017011541 & -6.14805425179248 \\
\hline 1.75181873697455 & -6.17032965630192 \\
\hline 5.29085265641775 & -7.09383881812695 \\
\hline 3.84285759754527 & -4.97254453006008 \\
\hline 1.92955649116175 & -7.00997978954392 \\
\hline 4.33569009148426 & 4.28267772615105 \\
\hline 7.43180788961624 & 2.97723130516403 \\
\hline 4.34261528639779 & 2.12130485301086 \\
\hline 6.40967398810477 & -0.24065809952543 \\
\hline 8.38160501909407 & 2.83978314548571 \\
\hline 5.91667918248583 & 2.11817399426502 \\
\hline 7.26315270531855 & -0.33108561071501 \\
\hline 0.00000000000000 & 0.00000000000000 \\
\hline 0.26953359201756 & -2.30262510281352 \\
\hline 0.67520298111439 & -5.13956276304090 \\
\hline-0.13134758203272 & -6.03192888790232 \\
\hline-0.46975143734054 & -6.85587129265764 \\
\hline 2.27321706910398 & -0.45520860939500 \\
\hline 5.09666385251493 & -0.94605604242557 \\
\hline 5.69611663965029 & -1.98888750901485 \\
\hline 6.37515989049258 & -2.56534090620030 \\
\hline 1.13539182049513 & 2.02129071023583 \\
\hline 2.47470850897284 & 4.55486797355969 \\
\hline 3.65174126922046 & 4.80272880753106 \\
\hline 4.40981693338003 & 5.27040341989538 \\
\hline-1.57150633348937 & 1.70443496946519 \\
\hline-3.56720988172116 & 3.76111926435381 \\
\hline-3.43921641715135 & 4.95713715081729 \\
\hline-3.64974314149890 & 5.82262935411931 \\
\hline-2.10663614812730 & -0.96789196749248 \\
\hline-4.67936546088097 & -2.23036843244703 \\
\hline-5.77729390968666 & -1.73904956143115 \\
\hline-6.66548224503320 & -1.67182057515677 \\
\hline 2.73326663803638 & -5.44690651295416 \\
\hline 0.67553887997517 & -4.78559181572194 \\
\hline 0.53496029647296 & -7.98808439075405 \\
\hline-2.20957764448027 & -6.02159477112727 \\
\hline 0.11073587434090 & -8.84892132271622 \\
\hline-2.55931874620588 & -6.80535762893607 \\
\hline 0.18614876224009 & -6.28164805260197 \\
\hline 6.02494177410684 & 0.91630436760218 \\
\hline 4.76012127494445 & -0.83635354538864 \\
\hline 7.76243153546830 & -1.95967635332446 \\
\hline 5.04407990303403 & -3.96220833456072 \\
\hline
\end{tabular}

$8.04140543887694 \mathrm{~h}$

$7.91589044850113 \mathrm{~h}$

$3.24897204489626 \mathrm{~h}$

$2.95499646136592 \mathrm{~h}$

$1.06051547261897 \mathrm{~h}$

$4.91414686797613 \mathrm{~h}$

$9.16036996777358 \mathrm{~h}$

$8.04140543887694 \mathrm{~h}$

$7.91589044850113 \mathrm{~h}$

$3.24897204489626 \mathrm{~h}$

$1.06051547261897 \mathrm{~h}$

$2.95499646136592 \mathrm{~h}$

$4.91414686797613 \mathrm{~h}$

$8.04140543887694 \mathrm{~h}$

$9.16036996777358 \mathrm{~h}$

$7.91589044850113 \mathrm{~h}$

$3.24897204489626 \mathrm{~h}$

$1.06051547261897 \mathrm{~h}$

$2.95499646136592 \mathrm{~h}$

$0.00000000000000 \mathrm{bk}$

$-4.63928549464807 \quad c$

-5.02243822940706 c

$-7.68868271597103 \mathrm{C}$

-2.96038272598829 c

$-4.63928549464807 \quad \mathrm{c}$

$-5.02243822940706 \quad c$

$-7.68868271597103 \mathrm{c}$

$-2.96038272598829 \quad \mathrm{c}$

$-4.63928549464807 \quad \mathrm{c}$

-5.02243822940706 c

$-7.68868271597103 \quad \mathrm{c}$

-2.96038272598829 c

$-4.63928549464807 \quad \mathrm{c}$

-5.02243822940706 c

$-7.68868271597103 \quad \mathrm{c}$

-2.96038272598829 c

$-4.63928549464807 \quad \mathrm{c}$

$-5.02243822940706 \quad c$

$-7.68868271597103 \mathrm{c}$

-2.96038272598829 c

$-4.91414686797613 \mathrm{~h}$

$-9.16036996777358 \mathrm{~h}$

$-8.04140543887694 \mathrm{~h}$

$-7.91589044850113 \mathrm{~h}$

$-3.24897204489626 \mathrm{~h}$

$-2.95499646136592 \mathrm{~h}$

$-1.06051547261897 \mathrm{~h}$

$-4.91414686797613 \mathrm{~h}$

$-9.16036996777358 \mathrm{~h}$

$-8.04140543887694 \mathrm{~h}$

$-7.91589044850113 \mathrm{~h}$ 


\begin{abstract}
8.45004355321067
5.68140673211858

6.03172544451392

0.99035215860079

2. 26637785851201

4. 26248622779044

5.32699046652556

5.11167824796019

6.07062121056762

3.54166257327707

$-5.41287047925977$

$-3.35942272703385$

$-5.12807017011541$

$-1.75181873697455$

$-5.29085265641775$

$-3.84285759754527$

$-1.92955649116175$

$-4.33569009148426$

$-7.43180788961624$

$-4.34261528639779$

$-6.40967398810477$

$-8.38160501909407$

$-5.91667918248583$

$-7.26315270531855$
\end{abstract}

\$end

\subsection{No}

\$coord

$\begin{array}{rr}-0.26619029509727 & 2.30336260363035 \\ -0.67068412390946 & 5.13934691191358 \\ 0.13221672570889 & 6.00300980715039 \\ 0.47494768123040 & 6.88859388651932 \\ -2.27288533849594 & 0.45861617400276 \\ -5.09506216222229 & 0.95028702934988 \\ -5.66834437928946 & 1.98077762635756 \\ -6.40467519894598 & 2.58039466541527 \\ -1.13853009662458 & -2.01992222030619 \\ -2.47823746713743 & -4.55203722870719 \\ -3.63544621204918 & -4.77882190990610 \\ -4.43325464108251 & -5.29382227890376 \\ 1.56923504156724 & -1.70699676078315 \\ 3.56342717533790 & -3.76360075474584 \\ 3.42151505597099 & -4.93425199286226 \\ 3.66477314997374 & -5.85215676417921 \\ 2.10837068865048 & 0.96494020345628 \\ 4.68055657793129 & 2.22600404218949 \\ 5.75005880965877 & 1.72928646926033 \\ 6.69820900882430 & 1.67699049114839 \\ -2.72912072344894 & 5.44886550777783\end{array}$

$-3.24897204489626 \mathrm{~h}$

$-2.95499646136592 \mathrm{~h}$

$-1.06051547261897 \mathrm{~h}$

$-4.91414686797613 \mathrm{~h}$

$-9.16036996777358 \mathrm{~h}$

$-8.04140543887694 \mathrm{~h}$

$-7.91589044850113 \mathrm{~h}$

$-3.24897204489626 \mathrm{~h}$

$-2.95499646136592 \mathrm{~h}$

$-1.06051547261897 \mathrm{~h}$

$-4.91414686797613 \mathrm{~h}$

$-9.16036996777358 \mathrm{~h}$

$-8.04140543887694 \mathrm{~h}$

$-7.91589044850113 \mathrm{~h}$

$-3.24897204489626 \mathrm{~h}$

$-1.06051547261897 \mathrm{~h}$

$-2.95499646136592 \mathrm{~h}$

$-4.91414686797613 \mathrm{~h}$

$-8.04140543887694 \mathrm{~h}$

$-9.16036996777358 \mathrm{~h}$

$-7.91589044850113 \mathrm{~h}$

$-3.24897204489626 \mathrm{~h}$

$-1.06051547261897 \mathrm{~h}$

$-2.95499646136592 \mathrm{~h}$

$4.61485031111617 \quad \mathrm{c}$

$5.02500453076042 \quad \mathrm{c}$

$7.70319789568155 \quad \mathrm{c}$

$2.98969509306159 \quad \mathrm{c}$

$4.61485031111617 \quad \mathrm{c}$

$5.02500453076042 \quad \mathrm{c}$

$7.70319789568155 \quad \mathrm{c}$

$2.98969509306159 \quad \mathrm{c}$

$4.61485031111617 \quad \mathrm{c}$

$5.02500453076042 \quad \mathrm{c}$

$7.70319789568155 \quad \mathrm{c}$

$2.98969509306159 \quad \mathrm{c}$

$4.61485031111617 \quad \mathrm{c}$

$5.02500453076042 \quad \mathrm{c}$

$7.70319789568155 \quad \mathrm{c}$

$2.98969509306159 \quad \mathrm{c}$

$4.61485031111617 \quad \mathrm{c}$

$5.02500453076042 \quad \mathrm{c}$

$7.70319789568155 \quad \mathrm{c}$

2.98969509306159 c

$4.92080519990904 \mathrm{~h}$ 


\begin{tabular}{rr}
-0.66339086286062 & 4.73388936004136 \\
-0.54889016034209 & 7.94954486156688 \\
2.21065109574349 & 6.00763390951390 \\
-0.12147210979017 & 8.87313352330847 \\
2.56555099216464 & 6.85683922971448 \\
-0.16569374872080 & 6.33597763357186 \\
-6.02552373083458 & -0.91175600582542 \\
-4.70719537382459 & 0.83193005876926 \\
-7.73008282976474 & 1.93451889604657 \\
-5.03047061994057 & 3.95891510388486 \\
-8.47638840356722 & 2.62642221049630 \\
-5.72844277409383 & 4.55886383866427 \\
-6.07707499972528 & 1.80034064532298 \\
-0.99485774222593 & -6.01236170882478 \\
-2.24581586984924 & -4.21972830745923 \\
-4.22856376430448 & -6.75394643193116 \\
-5.31965291827450 & -3.56088981673775 \\
-5.11722402545982 & -7.24991532841411 \\
-6.10592332916337 & -4.03930642733716 \\
-3.59014515329169 & -5.22330592343435 \\
5.41066783216798 & -2.80408788288671 \\
3.31920483378385 & -3.43986557606910 \\
5.11668669982834 & -6.10868734917610 \\
1.74274430809438 & -6.15966604082215 \\
5.31377002778551 & -7.10711629901506 \\
3.85824327044533 & -5.02852123964407 \\
1.95477462396996 & -7.05529250173455 \\
4.33883436434142 & 4.27934008975908 \\
7.39085005458294 & 2.97857002349384 \\
4.29719727275060 & 2.09377446471776 \\
6.39672813437725 & -0.24599315583887 \\
8.40131451103177 & 2.85747589362442 \\
5.97467063129244 & 2.11550888418353 \\
7.31404048712259 & -0.32110413930709 \\
0.00000000000000 & 0.00000000000000 \\
0.26619029509727 & -2.30336260363035 \\
0.67068412390946 & -5.13934691191358 \\
-0.13221672570889 & -6.00300980715039 \\
-0.47494768123040 & -6.88859388651932 \\
2.27288533849594 & -0.45861617400276 \\
5.09506216222229 & -0.95028702934988 \\
5.66834437928946 & -1.98077762635756 \\
6.40467519894598 & -2.58039466541527 \\
1.13853009662458 & 2.01992222030619 \\
2.47823746713743 & 4.55203722870719 \\
3.63544621204918 & 4.77882190990610 \\
4.43325464108251 & 5.29382227890376 \\
-3.56923504156724 & 1.70699676078315 \\
-3.66477731499737374 & 5.76360075474584 \\
\hline
\end{tabular}

$9.16140521728991 \mathrm{~h}$

$8.07996020897031 \mathrm{~h}$

$7.92905487585489 \mathrm{~h}$

$3.30516690644123 \mathrm{~h}$

$2.99742477059438 \mathrm{~h}$

$1.07800173678811 \mathrm{~h}$

$4.92080519990904 \mathrm{~h}$

$9.16140521728991 \mathrm{~h}$

$8.07996020897031 \mathrm{~h}$

$7.92905487585489 \mathrm{~h}$

$3.30516690644123 \mathrm{~h}$

$2.99742477059438 \mathrm{~h}$

$1.07800173678811 \mathrm{~h}$

$4.92080519990904 \mathrm{~h}$

$9.16140521728991 \mathrm{~h}$

$8.07996020897031 \mathrm{~h}$

$7.92905487585489 \mathrm{~h}$

$3.30516690644123 \mathrm{~h}$

$2.99742477059438 \mathrm{~h}$

$1.07800173678811 \mathrm{~h}$

$4.92080519990904 \mathrm{~h}$

$9.16140521728991 \mathrm{~h}$

$8.07996020897031 \mathrm{~h}$

$7.92905487585489 \mathrm{~h}$

$3.30516690644123 \mathrm{~h}$

$1.07800173678811 \mathrm{~h}$

$2.99742477059438 \mathrm{~h}$

$4.92080519990904 \mathrm{~h}$

$8.07996020897031 \mathrm{~h}$

$9.16140521728991 \mathrm{~h}$

$7.92905487585489 \mathrm{~h}$

$3.30516690644123 \mathrm{~h}$

$1.07800173678811 \mathrm{~h}$

$2.99742477059438 \mathrm{~h}$

0.00000000000000 no

$-4.61485031111617 \mathrm{c}$

$-5.02500453076042 \quad \mathrm{c}$

-7.70319789568155 c

-2.98969509306159 c

$-4.61485031111617 \quad \mathrm{c}$

$-5.02500453076042 \quad c$

-7.70319789568155 c

$-2.98969509306159 \quad \mathrm{c}$

$-4.61485031111617 \quad \mathrm{c}$

$-5.02500453076042 \quad c$

$-7.70319789568155 \quad \mathrm{c}$

$-2.98969509306159 \quad \mathrm{c}$

$-4.61485031111617 \quad \mathrm{c}$

$-5.02500453076042 \mathrm{c}$

$-7.70319789568155 \mathrm{c}$

-2.98969509306159 c 


\begin{abstract}
$-2.10837068865048$
$-4.68055657793129$

$-5.75005880965877$

$-6.69820900882430$

2.72912072344894

0.66339086286062

0.54889016034209

$-2.21065109574349$

0.12147210979017

$-2.56555099216464$

0.16569374872080

6.02552373083458

4.70719537382459

7.73008282976474

5.03047061994057

8.47638840356722

5.72844277409383

6.07707499972528

0.99485774222593

2. 24581586984924

4. 22856376430448

5.31965291827450

5. 11722402545982

6.10592332916337

3. 59014515329169

$-5.41066783216798$

$-3.31920483378385$

$-5.11668669982834$

$-1.74274430809438$

$-5.31377002778551$

$-3.85824327044533$

$-1.95477462396996$

$-4.33883436434142$

$-7.39085005458294$

$-4.29719727275060$

$-6.39672813437725$

$-8.40131451103177$

$-5.97467063129244$

$-7.31404048712259$
\end{abstract} \$end

\section{$\begin{array}{ll}1.7 & \mathrm{Lr}\end{array}$}

\$coord

$-0.26076714089121$

$-0.66518749557667$

0.11648712692024

0.49643031520497

$-2.26939217724545$

$-5.09247952810057$
$-0.96494020345628$

$-2.22600404218949$

$-1.72928646926033$

$-1.67699049114839$

$-5.44886550777783$

$-4.73388936004136$

$-7.94954486156688$

$-6.00763390951390$

$-8.87313352330847$

$-6.85683922971448$

$-6.33597763357186$

0.91175600582542

$-0.83193005876926$

$-1.93451889604657$

$-3.95891510388486$

$-2.62642221049630$

$-4.55886383866427$

$-1.80034064532298$

6.01236170882478

4. 21972830745923

6.75394643193116

3. 56088981673775

7.24991532841411

4.03930642733716

5. 22330592343435

2.80408788288671

3. 43986557606910

6.10868734917610

6.15966604082215

7.10711629901506

5.02852123964407

7.05529250173455

$-4.27934008975908$

$-2.97857002349384$

$-2.09377446471776$

0.24599315583887

$-2.85747589362442$

$-2.11550888418353$

0.32110413930709
$-4.61485031111617 \quad \mathrm{c}$

$-5.02500453076042 \mathrm{c}$

$-7.70319789568155 \mathrm{c}$

$-2.98969509306159 \mathrm{c}$

$-4.92080519990904 \mathrm{~h}$

$-9.16140521728991 \mathrm{~h}$

$-8.07996020897031 \mathrm{~h}$

$-7.92905487585489 \mathrm{~h}$

$-3.30516690644123 \mathrm{~h}$

$-2.99742477059438 \mathrm{~h}$

$-1.07800173678811 \mathrm{~h}$

$-4.92080519990904 \mathrm{~h}$

$-9.16140521728991 \mathrm{~h}$

$-8.07996020897031 \mathrm{~h}$

$-7.92905487585489 \mathrm{~h}$

$-3.30516690644123 \mathrm{~h}$

$-2.99742477059438 \mathrm{~h}$

$-1.07800173678811 \mathrm{~h}$

$-4.92080519990904 \mathrm{~h}$

$-9.16140521728991 \mathrm{~h}$

$-8.07996020897031 \mathrm{~h}$

$-7.92905487585489 \mathrm{~h}$

$-3.30516690644123 \mathrm{~h}$

$-2.99742477059438 \mathrm{~h}$

$-1.07800173678811 \mathrm{~h}$

$-4.92080519990904 \mathrm{~h}$

$-9.16140521728991 \mathrm{~h}$

$-8.07996020897031 \mathrm{~h}$

$-7.92905487585489 \mathrm{~h}$

$-3.30516690644123 \mathrm{~h}$

$-1.07800173678811 \mathrm{~h}$

$-2.99742477059438 \mathrm{~h}$

$-4.92080519990904 \mathrm{~h}$

$-8.07996020897031 \mathrm{~h}$

$-9.16140521728991 \mathrm{~h}$

$-7.92905487585489 \mathrm{~h}$

$-3.30516690644123 \mathrm{~h}$

$-1.07800173678811 \mathrm{~h}$

$-2.99742477059438 \mathrm{~h}$
2.30145176614956

5.13841733250376

5.97983938690901

6.90152028301767

0.46318341889418

0.95522737770820
$4.56828439589982 \quad \mathrm{c}$ $4.97460021307923 \quad \mathrm{c}$ $7.66602173507695 \quad \mathrm{c}$ $2.96275652333136 \quad \mathrm{c}$ 4.56828439589982 c $4.97460021307923 \quad \mathrm{c}$ 


$\begin{array}{rr}-5.65116871347399 & 1.95865783530957 \\ -6.41033043358586 & 2.60482034063800 \\ -1.14179435844959 & -2.01518867024756 \\ -2.48213793980249 & -4.54805434609565 \\ -3.60910146800718 & -4.76932227235643 \\ -4.45823240227889 & -5.29165277791629 \\ 1.56372445556074 & -1.70863851085087 \\ 3.55843391653695 & -3.76607954627701 \\ 3.42062133739837 & -4.90626110292771 \\ 3.65499127923141 & -5.87524161405306 \\ 2.10822922102554 & 0.95919199605470 \\ 4.68137104694275 & 2.22048918216070 \\ 5.72316171716252 & 1.73708615306552 \\ 6.71714124142837 & 1.66055376831368 \\ -2.72284992706765 & 5.44282831184500 \\ -0.67773625252457 & 4.69539397237143 \\ -0.57941162218633 & 7.91861487366365 \\ 2.19383816632146 & 5.99744297251651 \\ -0.14046448139625 & 8.87423859217399 \\ 2.58662873437945 & 6.90258012002174 \\ -0.09241141490318 & 6.32753659581692 \\ -6.01784423365241 & -0.90765772020626 \\ -4.67501705373092 & 0.80639105345554 \\ -7.71009831362351 & 1.89593336897486 \\ -5.02597394381866 & 3.93977588507926 \\ -8.48330835210252 & 2.60870087677987 \\ -5.76543156533426 & 4.59304467518926 \\ -6.04640160922706 & 1.86742786231312 \\ -0.99638234833211 & -6.00379163308369 \\ -2.21158318466656 & -4.19701689311206 \\ -4.18569119223622 & -6.74686361123208 \\ -5.30006089017281 & -3.56252756748032 \\ -5.10250841724895 & -7.26197278384240 \\ -6.14986140156752 & -4.06392239890807 \\ -3.64447028923120 & -5.17340270536888 \\ 5.40204607659271 & -2.80288957041170 \\ 3.30818347665922 & -3.40029014475631 \\ 5.12319889041041 & -6.06572439817617 \\ 1.75035617124787 & -6.14153900764057 \\ 5.32978472236021 & -7.09684688257114 \\ 3.79399509949301 & -5.06476657172171 \\ 1.96460819306444 & -7.10468684535646 \\ 4.33503043245942 & 4.27151061185667 \\ 7.35200223763567 & 2.99803976676974 \\ 4.25615301426276 & 2.09552201204141 \\ 6.38184049642208 & -0.23315228247487 \\ 8.39649652838751 & 2.87588019745971 \\ 5.98928821386843 & 2.04320481896055 \\ 7.36405603945789 & -0.32701555094648 \\ 0.00000000000000 & -0.00000000000000 \\ 0.26076714089121 & -2.30145176614956\end{array}$

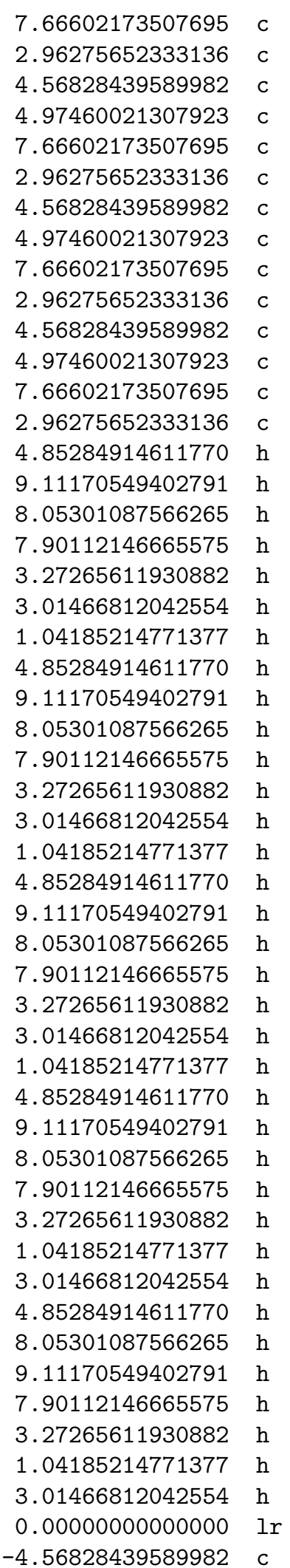




\begin{tabular}{|c|c|c|}
\hline 0.66518749557667 & -5.13841733250376 & -4.97460021307923 \\
\hline-0.11648712692024 & -5.97983938690901 & -7.66602173507695 \\
\hline-0.49643031520497 & -6.90152028301767 & -2.96275652333136 \\
\hline 2. 26939217724545 & -0.46318341889418 & -4.56828439589982 \\
\hline 5.09247952810057 & -0.95522737770820 & -4.97460021307923 \\
\hline 5.65116871347399 & -1.95865783530957 & -7.66602173507695 \\
\hline 6.41033043358586 & -2.60482034063800 & -2.96275652333136 \\
\hline 1.14179435844959 & 2.01518867024756 & -4.56828439589982 \\
\hline 2.48213793980249 & 4.54805434609565 & -4.97460021307923 \\
\hline 3.60910146800718 & 4.76932227235643 & -7.66602173507695 \\
\hline 4.45823240227889 & 5.29165277791629 & -2.96275652333136 \\
\hline-1.56372445556074 & 1.70863851085087 & -4.56828439589982 \\
\hline-3.55843391653695 & 3.76607954627701 & -4.97460021307923 \\
\hline-3.42062133739837 & 4.90626110292771 & -7.66602173507695 \\
\hline-3.65499127923141 & 5.87524161405306 & -2.96275652333136 \\
\hline-2.10822922102554 & -0.95919199605470 & -4.56828439589982 \\
\hline-4.68137104694275 & -2.22048918216070 & -4.97460021307923 \\
\hline-5.72316171716252 & -1.73708615306552 & -7.66602173507695 \\
\hline-6.71714124142837 & -1.66055376831368 & -2.96275652333136 \\
\hline 2.72284992706765 & -5.44282831184500 & -4.85284914611770 \\
\hline 0.67773625252457 & -4.69539397237143 & -9.11170549402791 \\
\hline 0.57941162218633 & -7.91861487366365 & -8.05301087566265 \\
\hline-2.19383816632146 & -5.99744297251651 & -7.90112146665575 \\
\hline 0.14046448139625 & -8.87423859217399 & -3.27265611930882 \\
\hline-2.58662873437945 & -6.90258012002174 & -3.01466812042554 \\
\hline 0.09241141490318 & -6.32753659581692 & -1.04185214771377 \\
\hline 6.01784423365241 & 0.90765772020626 & -4.85284914611770 \\
\hline 4.67501705373092 & -0.80639105345554 & -9.11170549402791 \\
\hline 7.71009831362351 & -1.89593336897486 & -8.05301087566265 \\
\hline 5.02597394381866 & -3.93977588507926 & -7.90112146665575 \\
\hline 8.48330835210252 & -2.60870087677987 & -3.27265611930882 \\
\hline 5.76543156533426 & -4.59304467518926 & -3.01466812042554 \\
\hline 6.04640160922706 & -1.86742786231312 & -1.04185214771377 \\
\hline 0.99638234833211 & 6.00379163308369 & -4.85284914611770 \\
\hline 2.21158318466656 & 4.19701689311206 & -9.11170549402791 \\
\hline 4.18569119223622 & 6.74686361123208 & -8.05301087566265 \\
\hline 5.30006089017281 & 3.56252756748032 & -7.90112146665575 \\
\hline 5.10250841724895 & 7.26197278384240 & -3.27265611930882 \\
\hline 6.14986140156752 & 4.06392239890807 & -3.01466812042554 \\
\hline 3.64447028923120 & 5.17340270536888 & -1.04185214771377 \\
\hline-5.40204607659271 & 2.80288957041170 & -4.85284914611770 \\
\hline-3.30818347665922 & 3.40029014475631 & -9.11170549402791 \\
\hline-5.12319889041041 & 6.06572439817617 & -8.05301087566265 \\
\hline-1.75035617124787 & 6.14153900764057 & -7.90112146665575 \\
\hline-5.32978472236021 & 7.09684688257114 & -3.27265611930882 \\
\hline-3.79399509949301 & 5.06476657172171 & -1.04185214771377 \\
\hline-1.96460819306444 & 7.10468684535646 & -3.01466812042554 \\
\hline-4.33503043245942 & -4.27151061185667 & -4.85284914611770 \\
\hline-7.35200223763567 & -2.99803976676974 & -8.05301087566265 \\
\hline-4.25615301426276 & -2.09552201204141 & -9.11170549402791 \\
\hline-6.38184049642208 & 0.23315228247487 & -7.90112146665575 \\
\hline
\end{tabular}




$\begin{array}{lrrr}-8.39649652838751 & -2.87588019745971 & -3.27265611930882 & \mathrm{~h} \\ -5.98928821386843 & -2.04320481896055 & -1.04185214771377 & \mathrm{~h} \\ -7.36405603945789 & 0.32701555094648 & -3.01466812042554 & \mathrm{~h} \\ \text { \$end } & & & \end{array}$

\section{Orbital occupations for $\mathrm{An}\left(\mathrm{Cp}^{\mathrm{iPr}}\right)_{2}$}

\subsection{Th}

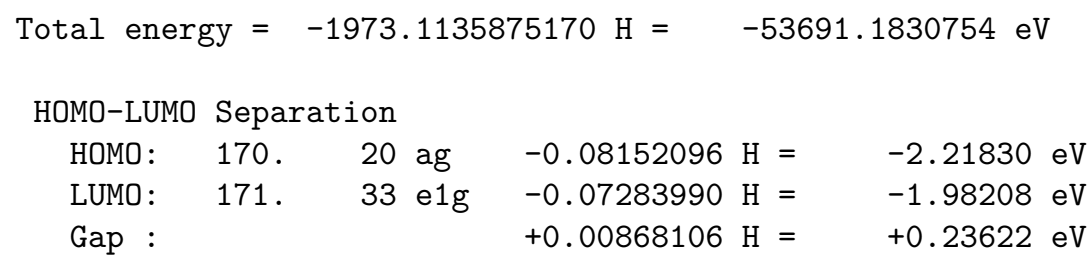

Number of MOs $=799$, Electrons $=340.00$, Symmetry: s10

\begin{tabular}{|c|c|c|c|c|}
\hline Nr. & Orbital & Occupation & Energy & \\
\hline 187. & $36 \mathrm{e} 2 \mathrm{~g}$ & & $+0.044011 \mathrm{H}=$ & $+1.198 \mathrm{el}$ \\
\hline 186. & 35 e $2 g$ & & $+0.044011 \mathrm{H}=$ & $+1.198 \mathrm{el}$ \\
\hline 185. & 36 e $2 u$ & & $+0.041532 \mathrm{H}=$ & $+1.130 \mathrm{el}$ \\
\hline 184. & 35 e $2 u$ & & $+0.041532 \mathrm{H}=$ & $+1.130 \mathrm{e}$ \\
\hline 183. & $20 \mathrm{au}$ & & $+0.036828 \mathrm{H}=$ & $+1.002 \mathrm{el}$ \\
\hline 182. & 40 e1u & & $+0.028168 \mathrm{H}=$ & $+0.766 \mathrm{el}$ \\
\hline 181. & 39 e1u & & $+0.028168 \mathrm{H}=$ & $+0.766 \mathrm{el}$ \\
\hline 180. & $21 \mathrm{ag}$ & & $+0.023069 \mathrm{H}=$ & $+0.628 \mathrm{e}$ \\
\hline 179. & $38 \mathrm{e} 1 \mathrm{u}$ & & $-0.028247 \mathrm{H}=$ & $-0.769 \mathrm{el}$ \\
\hline 178. & $37 \mathrm{e} 1 \mathrm{u}$ & & $-0.028247 \mathrm{H}=$ & $-0.769 \mathrm{el}$ \\
\hline 177. & $19 \mathrm{au}$ & & $-0.037805 \mathrm{H}=$ & $-1.029 \mathrm{el}$ \\
\hline 176. & $34 \mathrm{e} 2 \mathrm{u}$ & & $-0.047151 \mathrm{H}=$ & $-1.283 \mathrm{el}$ \\
\hline 175. & 33 e $2 u$ & & $-0.047151 \mathrm{H}=$ & $-1.283 \mathrm{el}$ \\
\hline 174. & 32 e $2 u$ & & $-0.055526 \mathrm{H}=$ & $-1.511 \mathrm{eV}$ \\
\hline 173. & $31 \mathrm{e} 2 \mathrm{u}$ & & $-0.055526 \mathrm{H}=$ & $-1.511 \mathrm{el}$ \\
\hline 172. & $34 \mathrm{e} 1 \mathrm{~g}$ & & $-0.072840 \mathrm{H}=$ & $-1.982 \mathrm{el}$ \\
\hline 171. & $33 \mathrm{e} 1 \mathrm{~g}$ & & $-0.072840 \mathrm{H}=$ & $-1.982 \mathrm{el}$ \\
\hline 170. & $20 \mathrm{ag}$ & 2.000 & $-0.081521 \mathrm{H}=$ & $-2.218 \mathrm{el}$ \\
\hline 169. & $36 \mathrm{e} 1 \mathrm{u}$ & 2.000 & $-0.176497 \mathrm{H}=$ & $-4.803 \mathrm{el}$ \\
\hline 168. & $35 \mathrm{e} 1 \mathrm{u}$ & 2.000 & $-0.176497 \mathrm{H}=$ & -4.803 \\
\hline 167. & $34 \mathrm{e} 2 \mathrm{~g}$ & 2.000 & $-0.197981 \mathrm{H}=$ & $-5.387 \mathrm{el}$ \\
\hline 166. & 33 e2g & 2.000 & $-0.197981 \mathrm{H}=$ & $-5.387 \mathrm{el}$ \\
\hline 165. & 30 e $2 u$ & 2.000 & $-0.255383 \mathrm{H}=$ & $-6.949 \mathrm{el}$ \\
\hline 164. & 29 e $2 u$ & 2.000 & $-0.255383 \mathrm{H}=$ & $-6.949 \mathrm{el}$ \\
\hline 163. & $31 \mathrm{e} 1 \mathrm{~g}$ & 2.000 & $-0.255505 \mathrm{H}=$ & $-6.953 \mathrm{el}$ \\
\hline 162. & $32 \mathrm{e} 1 \mathrm{~g}$ & 2.000 & $-0.255505 \mathrm{H}=$ & $-6.953 \mathrm{eI}$ \\
\hline 161. & $18 \mathrm{au}$ & 2.000 & $-0.265685 \mathrm{H}=$ & $-7.230 \mathrm{el}$ \\
\hline 160. & 32 e2g & 2.000 & $-0.281024 \mathrm{H}=$ & $-7.647 \mathrm{el}$ \\
\hline 159. & $31 \mathrm{e} 2 \mathrm{~g}$ & 2.000 & $-0.281024 \mathrm{H}=$ & $-7.647 \mathrm{el}$ \\
\hline & $34 \mathrm{e} 1 \mathrm{u}$ & 2.000 & $-0.281640 \mathrm{H}=$ & $-7.664 \mathrm{el}$ \\
\hline
\end{tabular}




$\begin{array}{lllll}\text { 157. } & 33 \mathrm{e} 1 \mathrm{u} & 2.000 & -0.281640 \mathrm{H}= & -7.664 \mathrm{eV} \\ 156 . & 19 \mathrm{ag} & 2.000 & -0.288309 \mathrm{H}= & -7.845 \mathrm{eV} \\ 155 . & 28 \mathrm{e} 2 \mathrm{u} & 2.000 & -0.293911 \mathrm{H}= & -7.998 \mathrm{eV}\end{array}$

\section{$2.2 \quad \mathrm{U}$}

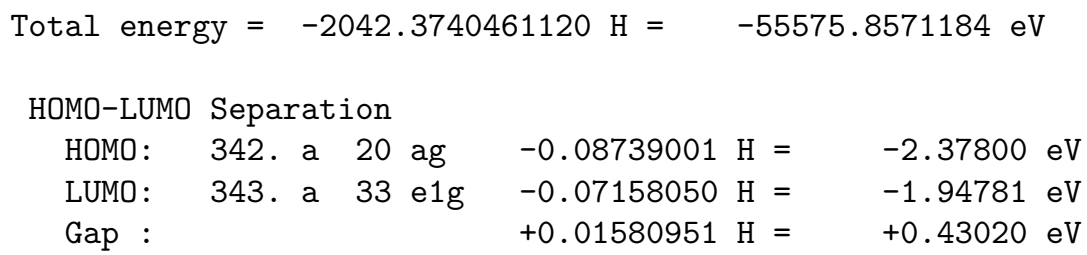

Number of $\mathrm{MOs}=1598$, Electrons $=342.00$, Symmetry: $\mathrm{s} 10$

\begin{tabular}{|c|c|c|c|c|c|c|c|}
\hline Nr. & Orbi & ital & Occupation & Energy & & & \\
\hline 359. a & 21 & ag & & $+0.024555 \mathrm{H}$ & {$[=$} & +0.668 & $\mathrm{eV}$ \\
\hline 358. b & 19 & $\mathrm{au}$ & & $+0.011521 \mathrm{H}$ & {$[=$} & +0.314 & $\mathrm{eV}$ \\
\hline $357 . \mathrm{b}$ & 38 & e1u & & $+0.000660 \mathrm{H}$ & {$[=$} & +0.018 & $\mathrm{eV}$ \\
\hline 356. b & 37 & e1u & & $+0.000660 \mathrm{H}$ & {$[=$} & +0.018 & $\mathrm{eV}$ \\
\hline 355. b & 34 & $\mathrm{e} 2 \mathrm{u}$ & & $-0.008633 \mathrm{H}$ & {$[=$} & -0.235 & $\mathrm{eV}$ \\
\hline $354 . \mathrm{b}$ & 33 & $\mathrm{e} 2 \mathrm{u}$ & & $-0.008633 \mathrm{H}$ & {$[=$} & -0.235 & $\mathrm{eV}$ \\
\hline 353. b & 32 & $\mathrm{e} 2 \mathrm{u}$ & & $-0.023552 \mathrm{H}$ & {$[=$} & -0.641 & $\mathrm{eV}$ \\
\hline 352. b & 31 & $\mathrm{e} 2 \mathrm{u}$ & & $-0.023552 \mathrm{H}$ & {$[=$} & -0.641 & $\mathrm{eV}$ \\
\hline $351 . b$ & 34 & e1g & & $-0.033921 \mathrm{H}$ & {$[=$} & -0.923 & $\mathrm{eV}$ \\
\hline 350. b & 33 & e1g & & $-0.033921 \mathrm{H}$ & $I=$ & -0.923 & $\mathrm{eV}$ \\
\hline 349. a & 38 & e1u & & $-0.034585 \mathrm{H}$ & {$[=$} & -0.941 & $\mathrm{eV}$ \\
\hline 348. a & 37 & e1u & & $-0.034585 \mathrm{H}$ & {$[=$} & -0.941 & $\mathrm{eV}$ \\
\hline $347 . \mathrm{b}$ & 20 & $\mathrm{ag}$ & & $-0.036880 \mathrm{H}$ & {$[=$} & -1.004 & $\mathrm{eV}$ \\
\hline 346. a & 34 & $\mathrm{e} 2 \mathrm{u}$ & & $-0.051411 \mathrm{H}$ & {$[=$} & -1.399 & $\mathrm{eV}$ \\
\hline 345. a & 33 & $\mathrm{e} 2 \mathrm{u}$ & & $-0.051411 \mathrm{H}$ & {$[=$} & -1.399 & $\mathrm{eV}$ \\
\hline 344. a & 34 & e1g & & $-0.071581 \mathrm{H}$ & {$[=$} & -1.948 & $\mathrm{eV}$ \\
\hline 343. a & 33 & e1g & & $-0.071581 \mathrm{H}$ & {$[=$} & -1.948 & $\mathrm{eV}$ \\
\hline 342. a & 20 & $\mathrm{ag}$ & 1.000 & $-0.087390 \mathrm{H}$ & {$[=$} & -2.378 & $\mathrm{eV}$ \\
\hline 341. a & 19 & $\mathrm{au}$ & 1.000 & $-0.091590 \mathrm{H}$ & {$[=$} & -2.492 & $\mathrm{eV}$ \\
\hline 340. a & 32 & $\mathrm{e} 2 \mathrm{u}$ & 1.000 & $-0.101634 \mathrm{H}$ & {$[=$} & -2.766 & $\mathrm{eV}$ \\
\hline 339. a & 31 & $\mathrm{e} 2 \mathrm{u}$ & 1.000 & $-0.101634 \mathrm{H}$ & {$[=$} & -2.766 & $\mathrm{eV}$ \\
\hline $338 . \mathrm{b}$ & 36 & e1u & 1.000 & $-0.167119 \mathrm{H}$ & {$[=$} & -4.548 & $\mathrm{eV}$ \\
\hline $337 . \mathrm{b}$ & 35 & e1u & 1.000 & $-0.167119 \mathrm{H}$ & {$[=$} & -4.548 & $\mathrm{eV}$ \\
\hline 336. a & 36 & e1u & 1.000 & $-0.169877 \mathrm{H}$ & $I=$ & -4.623 & $\mathrm{eV}$ \\
\hline 335. a & 35 & $\mathrm{e} 1 \mathrm{u}$ & 1.000 & $-0.169877 \mathrm{H}$ & {$[=$} & -4.623 & $\mathrm{eV}$ \\
\hline $334 . \mathrm{b}$ & 34 & e2g & 1.000 & $-0.186330 \mathrm{H}$ & {$[=$} & -5.070 & $\mathrm{eV}$ \\
\hline 333. b & 33 & $\mathrm{e} 2 \mathrm{~g}$ & 1.000 & $-0.186330 \mathrm{H}$ & {$[=$} & -5.070 & $\mathrm{eV}$ \\
\hline 332. a & 34 & $\mathrm{e} 2 \mathrm{~g}$ & 1.000 & $-0.191226 \mathrm{H}$ & {$[=$} & -5.204 & $\mathrm{eV}$ \\
\hline 331. a & 33 & e2g & 1.000 & $-0.191226 \mathrm{H}$ & {$[=$} & -5.204 & $\mathrm{eV}$ \\
\hline $330 . \mathrm{b}$ & 30 & $\mathrm{e} 2 \mathrm{u}$ & 1.000 & $-0.252815 \mathrm{H}$ & {$[=$} & -6.879 & $\mathrm{eV}$ \\
\hline 329. b & 29 & $\mathrm{e} 2 \mathrm{u}$ & 1.000 & $-0.252815 \mathrm{H}$ & {$[=$} & -6.879 & $\mathrm{eV}$ \\
\hline 328. b & 32 & e1g & 1.000 & $-0.252897 \mathrm{H}$ & {$[=$} & -6.882 & $\mathrm{eV}$ \\
\hline $327 . \mathrm{b}$ & 31 & e1g & 1.000 & $-0.252897 \mathrm{H}$ & {$[=$} & -6.882 & $\mathrm{eV}$ \\
\hline
\end{tabular}




\section{$2.3 \mathrm{Pu}$}

\begin{tabular}{|c|c|c|c|c|c|c|c|}
\hline \multicolumn{8}{|c|}{ HOMO-LUMO Separation } \\
\hline HOMO: & 344. & a 175 & \multicolumn{2}{|c|}{$-0.07708092 \mathrm{H}=$} & \multicolumn{3}{|c|}{$-2.09748 \mathrm{eV}$} \\
\hline LUMO: & 345 . & a 176 & -0.06 & $-0.06559138 \mathrm{H}=$ & \multicolumn{3}{|c|}{$-1.78483 \mathrm{eV}$} \\
\hline Gap : & & & \multicolumn{2}{|c|}{$+0.01148955 \mathrm{H}=$} & \multicolumn{3}{|c|}{$+0.31265 \mathrm{eV}$} \\
\hline Number of & $\mathrm{MOs}=$ & 1598, & , Electrons= & 344.00 & Symm & \multicolumn{2}{|l|}{$\mathrm{c} 1$} \\
\hline $\mathrm{Nr}$. & Orbital & & ccupation & Energy & & & \\
\hline $361 . \mathrm{b} 1$ & 179 a & & & +0.033855 & $\mathrm{H}=$ & $+0.921 \mathrm{e}$ & \\
\hline 360. a 1 & 182 a & & & +0.032374 & $\mathrm{H}=$ & $+0.881 \mathrm{e}$ & $\mathrm{eV}$ \\
\hline 359. b 1 & 178 a & & & +0.030357 & $\mathrm{H}=$ & $+0.826 \mathrm{e}$ & \\
\hline 358. a 1 & 181 a & & & +0.028358 & $\mathrm{H}=$ & $+0.772 \mathrm{e}$ & \\
\hline 357. b 1 & 177 a & & & +0.024536 & $\mathrm{H}=$ & $+0.668 \mathrm{e}$ & \\
\hline 356. b 1 & 176 a & & & +0.023644 & $\mathrm{H}=$ & $+0.643 \mathrm{e}$ & \\
\hline 355. a 1 & $180 \mathrm{a}$ & & & +0.023608 & $\mathrm{H}=$ & $+0.642 \mathrm{e}$ & $\mathrm{eV}$ \\
\hline 354. b 1 & 175 a & & & +0.010078 & $\mathrm{H}=$ & $+0.274 \mathrm{e}$ & \\
\hline 353. b 1 & 174 a & & & +0.003631 & $\mathrm{H}=$ & $+0.099 \mathrm{e}$ & \\
\hline 352. b 1 & 173 a & & & +0.003491 & $\mathrm{H}=$ & $+0.095 \mathrm{e}$ & $\mathrm{eV}$ \\
\hline 351. b 1 & $172 \mathrm{a}$ & & & -0.015595 & $\mathrm{H}=$ & $-0.424 \mathrm{e}$ & \\
\hline 350. b 1 & 171 a & & & -0.016845 & $\mathrm{H}=$ & -0.458 e & \\
\hline 349. b 1 & $170 \mathrm{a}$ & & & -0.030095 & $\mathrm{H}=$ & -0.819 e & \\
\hline 348. a 1 & 179 a & & & -0.044965 & $\mathrm{H}=$ & $-1.224 \mathrm{e}$ & \\
\hline 347 . a 1 & 178 a & & & -0.047475 & $\mathrm{H}=$ & $-1.292 \mathrm{e}$ & \\
\hline 346. a 1 & 177 a & & & -0.063268 & $\mathrm{H}=$ & $-1.722 \mathrm{e}$ & \\
\hline 345. a 1 & 176 a & & & -0.065591 & $\mathrm{H}=$ & -1.785 e & $\mathrm{eV}$ \\
\hline 344. a 1 & $175 \mathrm{a}$ & & 1.000 & -0.077081 & $\mathrm{H}=$ & $-2.097 \mathrm{e}$ & \\
\hline 343. a 1 & 174 a & & 1.000 & -0.081566 & $\mathrm{H}=$ & $-2.220 \mathrm{e}$ & \\
\hline 342. a 1 & 173 a & & 1.000 & -0.083530 & $\mathrm{H}=$ & $-2.273 \mathrm{e}$ & $\mathrm{eV}$ \\
\hline 341. a 1 & $172 \mathrm{a}$ & & 1.000 & -0.089621 & $\mathrm{H}=$ & $-2.439 e$ & \\
\hline 340. a 1 & 171 a & & 1.000 & -0.100955 & $\mathrm{H}=$ & $-2.747 \mathrm{e}$ & \\
\hline 339. a 1 & $170 \mathrm{a}$ & & 1.000 & -0.113872 & $\mathrm{H}=$ & -3.099 e & \\
\hline 338. b 1 & 169 a & & 1.000 & -0.161213 & $\mathrm{H}=$ & $-4.387 e$ & \\
\hline $337 . \quad b \quad 1$ & 168 a & & 1.000 & -0.161385 & $\mathrm{H}=$ & $-4.391 e$ & \\
\hline 336. a 1 & 169 a & & 1.000 & -0.166227 & $\mathrm{H}=$ & $-4.523 e$ & \\
\hline 335. a 1 & $168 \mathrm{a}$ & & 1.000 & -0.166405 & $\mathrm{H}=$ & $-4.528 e$ & $\mathrm{eV}$ \\
\hline 334. b 1 & $167 \mathrm{a}$ & & 1.000 & -0.176734 & $\mathrm{H}=$ & $-4.809 \mathrm{e}$ & \\
\hline 333. b 1 & $166 \mathrm{a}$ & & 1.000 & -0.178779 & $\mathrm{H}=$ & $-4.865 e$ & \\
\hline 332. a 1 & $167 \mathrm{a}$ & & 1.000 & -0.181811 & $\mathrm{H}=$ & $-4.947 e$ & $\mathrm{eV}$ \\
\hline 331 . a 1 & $166 a$ & & 1.000 & -0.183267 & $\mathrm{H}=$ & $-4.987 \mathrm{e}$ & \\
\hline $330 . \mathrm{b} 1$ & $165 \mathrm{a}$ & & 1.000 & -0.250091 & $\mathrm{H}=$ & $-6.805 e$ & \\
\hline 329. a 1 & 165 a & & 1.000 & -0.250157 & $\mathrm{H}=$ & $-6.807 \mathrm{e}$ & $\mathrm{eV}$ \\
\hline
\end{tabular}




\section{$2.4 \quad \mathrm{Am}$}

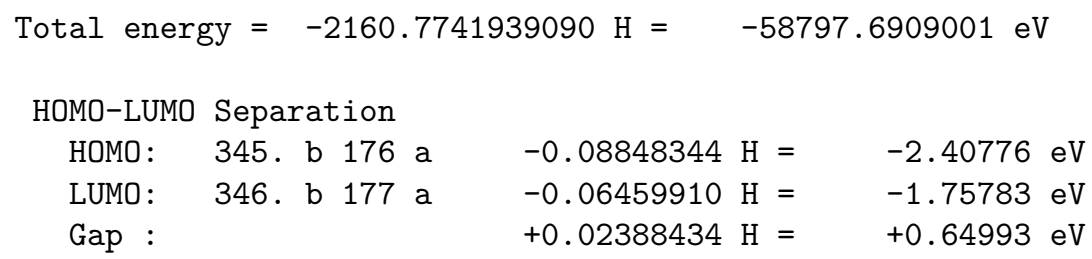

\section{$2.5 \quad \mathrm{Bk}$}

Total energy $=-2250.2473209060 \mathrm{H}=-61232.3799481 \mathrm{eV}$ 


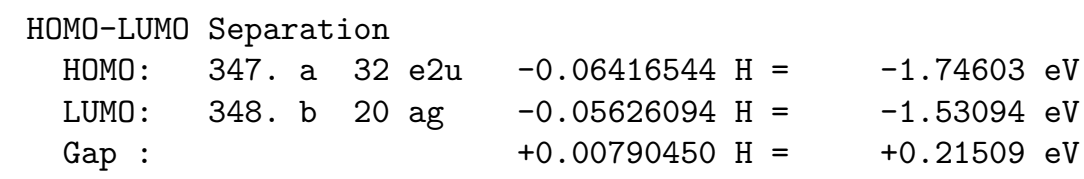

Number of MOs $=1598$, Electrons $=347.00$, Symmetry: $\mathrm{s} 10$

\begin{tabular}{|c|c|c|c|c|}
\hline $\mathrm{Nr}$. & Orbital & Occupation & Energy & \\
\hline 364. a & $21 \mathrm{ag}$ & & $+0.035400 \mathrm{H}=$ & $+0.963 \mathrm{eV}$ \\
\hline 363. b & $21 \mathrm{ag}$ & & $+0.033389 \mathrm{H}=$ & $+0.909 \mathrm{eV}$ \\
\hline 362. a & 40 e $1 u$ & & $+0.032910 \mathrm{H}=$ & $+0.896 \mathrm{eV}$ \\
\hline 361 . a & 39 e1u & & $+0.032910 \mathrm{H}=$ & $+0.896 \mathrm{eV}$ \\
\hline $360 . \mathrm{b}$ & 40 e1u & & $+0.031213 \mathrm{H}=$ & $+0.849 \mathrm{eV}$ \\
\hline 359. b & 39 e1u & & $+0.031213 \mathrm{H}=$ & $+0.849 \mathrm{eV}$ \\
\hline 358. a & $34 \mathrm{e} 2 \mathrm{u}$ & & $-0.000156 \mathrm{H}=$ & $-0.004 \mathrm{eV}$ \\
\hline 357. a & $33 \mathrm{e} 2 \mathrm{u}$ & & $-0.000156 \mathrm{H}=$ & $-0.004 \mathrm{eV}$ \\
\hline 356. a & $34 \mathrm{e} 1 \mathrm{~g}$ & & $-0.019213 \mathrm{H}=$ & $-0.523 \mathrm{eV}$ \\
\hline 355. a & $33 \mathrm{e} 1 \mathrm{~g}$ & & $-0.019213 \mathrm{H}=$ & $-0.523 \mathrm{eV}$ \\
\hline 354. a & 38 e1u & & $-0.020321 \mathrm{H}=$ & $-0.553 \mathrm{eV}$ \\
\hline 353. a & $37 \mathrm{e} 1 \mathrm{u}$ & & $-0.020321 \mathrm{H}=$ & $-0.553 \mathrm{eV}$ \\
\hline 352. b & $34 \mathrm{e} 1 \mathrm{~g}$ & & $-0.030807 \mathrm{H}=$ & $-0.838 \mathrm{eV}$ \\
\hline $351 . b$ & $33 \mathrm{e} 1 \mathrm{~g}$ & & $-0.030807 \mathrm{H}=$ & $-0.838 \mathrm{eV}$ \\
\hline 350. a & $20 \mathrm{ag}$ & & $-0.038743 \mathrm{H}=$ & $-1.054 \mathrm{eV}$ \\
\hline 349. a & $19 \mathrm{au}$ & & $-0.042671 \mathrm{H}=$ & $-1.161 \mathrm{eV}$ \\
\hline 348. b & $20 \mathrm{ag}$ & & $-0.056261 \mathrm{H}=$ & $-1.531 \mathrm{eV}$ \\
\hline 347 a & $32 \mathrm{e} 2 \mathrm{u}$ & 1.000 & $-0.064165 \mathrm{H}=$ & $-1.746 \mathrm{eV}$ \\
\hline 346. a & $31 \mathrm{e} 2 \mathrm{u}$ & 1.000 & $-0.064165 \mathrm{H}=$ & $-1.746 \mathrm{eV}$ \\
\hline 345. b & $34 \mathrm{e} 2 \mathrm{u}$ & 1.000 & $-0.124161 \mathrm{H}=$ & $-3.379 \mathrm{eV}$ \\
\hline $344 . \mathrm{b}$ & 33 e $2 u$ & 1.000 & $-0.124161 \mathrm{H}=$ & $-3.379 \mathrm{eV}$ \\
\hline 343. b & 38 e1u & 1.000 & $-0.136277 \mathrm{H}=$ & $-3.708 \mathrm{eV}$ \\
\hline 342. b & 37 e1u & 1.000 & $-0.136277 \mathrm{H}=$ & $-3.708 \mathrm{eV}$ \\
\hline $341 . b$ & 32 e $2 u$ & 1.000 & $-0.146864 \mathrm{H}=$ & $-3.996 \mathrm{eV}$ \\
\hline $340 . \mathrm{b}$ & $31 \mathrm{e} 2 \mathrm{u}$ & 1.000 & $-0.146864 \mathrm{H}=$ & $-3.996 \mathrm{eV}$ \\
\hline 339. a & 36 e $1 u$ & 1.000 & $-0.160563 \mathrm{H}=$ & $-4.369 \mathrm{eV}$ \\
\hline 338. a & $35 \mathrm{e} 1 \mathrm{u}$ & 1.000 & $-0.160563 \mathrm{H}=$ & $-4.369 \mathrm{eV}$ \\
\hline $337 . \mathrm{b}$ & $19 \mathrm{au}$ & 1.000 & $-0.166863 \mathrm{H}=$ & $-4.541 \mathrm{eV}$ \\
\hline 336. a & $33 \mathrm{e} 2 \mathrm{~g}$ & 1.000 & $-0.176625 \mathrm{H}=$ & $-4.806 \mathrm{eV}$ \\
\hline 335. a & $34 \mathrm{e} 2 \mathrm{~g}$ & 1.000 & $-0.176625 \mathrm{H}=$ & $-4.806 \mathrm{eV}$ \\
\hline $334 . \mathrm{b}$ & $34 \mathrm{e} 2 \mathrm{~g}$ & 1.000 & $-0.178338 \mathrm{H}=$ & $-4.853 \mathrm{eV}$ \\
\hline 333. b & $33 \mathrm{e} 2 \mathrm{~g}$ & 1.000 & $-0.178338 \mathrm{H}=$ & $-4.853 \mathrm{eV}$ \\
\hline 332. b & 36 e $1 \mathrm{u}$ & 1.000 & $-0.179961 \mathrm{H}=$ & $-4.897 \mathrm{eV}$ \\
\hline
\end{tabular}

\section{$2.6 \quad$ No}

Total energy $=-2514.2278750010 \mathrm{H}=-68415.6603978 \mathrm{eV}$

HOMO-LUMO Separation

HOMO: 176 . $38 \mathrm{e} 1 \mathrm{u} \quad-0.14096630 \mathrm{H}=\quad-3.83589 \mathrm{eV}$ 


\begin{tabular}{|c|c|c|c|c|c|c|}
\hline $\begin{array}{l}\text { LUMO: } \\
\text { Gap : }\end{array}$ & 177. & $\begin{array}{l}-0.05 \\
+0.08\end{array}$ & $\begin{array}{l}71575 \mathrm{H}= \\
25055 \mathrm{H}=\end{array}$ & $\begin{array}{l}-1.434 \\
+2.4014\end{array}$ & $\begin{array}{ll} \pm 7 & \mathrm{eV} \\
\pm 2 \mathrm{eV}\end{array}$ & \\
\hline Number of & $\mathrm{MO}=$ & 799, Electrons= & 352.00, & Symmetry: & s10 & \\
\hline $\mathrm{Nr}$. & Orbital & Occupation & Energy & & & \\
\hline 193. & 38 e $2 u$ & & $+0.074318 \mathrm{I}$ & $\mathrm{H}=$ & $+2.022 \mathrm{e}$ & $\mathrm{eV}$ \\
\hline 192. & $37 \mathrm{e} 2 \mathrm{u}$ & & $+0.074318 \mathrm{I}$ & $\mathrm{H}=$ & $+2.022 \mathrm{e}$ & $\mathrm{eV}$ \\
\hline 191. & $21 \mathrm{au}$ & & $+0.071189 \mathrm{H}$ & $\mathrm{H}=$ & $+1.937 \mathrm{e}$ & $\mathrm{eV}$ \\
\hline 190. & 36 e $1 \mathrm{~g}$ & & $+0.064125 \mathrm{~F}$ & $\mathrm{H}=$ & $+1.745 \mathrm{e}$ & $\mathrm{eV}$ \\
\hline 189. & $35 \mathrm{e} 1 \mathrm{~g}$ & & $+0.064125 \mathrm{~F}$ & $\mathrm{H}=$ & $+1.745 \mathrm{e}$ & $\mathrm{eV}$ \\
\hline 188. & $22 \mathrm{ag}$ & & $+0.062293 \mathrm{I}$ & $\mathrm{H}=$ & $+1.695 \mathrm{e}$ & $\mathrm{eV}$ \\
\hline 187. & $36 \mathrm{e} 2 \mathrm{~g}$ & & $+0.050847 \mathrm{H}$ & $\mathrm{H}=$ & $+1.384 \mathrm{e}$ & $\mathrm{eV}$ \\
\hline 186. & $35 \mathrm{e} 2 \mathrm{~g}$ & & $+0.050847 \mathrm{I}$ & $\mathrm{H}=$ & $+1.384 \mathrm{e}$ & $\mathrm{eV}$ \\
\hline 185. & $20 \mathrm{au}$ & & $+0.041115 \mathrm{I}$ & $\mathrm{H}=$ & $+1.119 \mathrm{e}$ & $\mathrm{eV}$ \\
\hline 184. & $21 \mathrm{ag}$ & & $+0.038646 \mathrm{H}$ & $\mathrm{H}=$ & $+1.052 \mathrm{e}$ & $\mathrm{eV}$ \\
\hline 183. & $36 \mathrm{e} 2 \mathrm{u}$ & & $+0.032353 \mathrm{H}$ & $\mathrm{H}=$ & $+0.880 \mathrm{e}$ & $\mathrm{eV}$ \\
\hline 182. & $35 \mathrm{e} 2 \mathrm{u}$ & & $+0.032353 \mathrm{I}$ & $\mathrm{H}=$ & $+0.880 \mathrm{e}$ & $\mathrm{eV}$ \\
\hline 181. & 40 e1u & & +0.031385 & $\mathrm{H}=$ & $+0.854 \mathrm{e}$ & $\mathrm{eV}$ \\
\hline 180. & 39 e1u & & $+0.031385 \mathrm{H}$ & $\mathrm{H}=$ & $+0.854 \mathrm{e}$ & $\mathrm{eV}$ \\
\hline 179. & $33 \mathrm{e} 1 \mathrm{~g}$ & & -0.017863 & $\mathrm{H}=$ & $-0.486 \mathrm{e}$ & $\mathrm{eV}$ \\
\hline 178 . & $34 \mathrm{e} 1 \mathrm{~g}$ & & -0.017863 & $\mathrm{H}=$ & $-0.486 \mathrm{e}$ & $\mathrm{eV}$ \\
\hline 177. & $20 \mathrm{ag}$ & & -0.052716 & $\mathrm{H}=$ & $-1.434 \mathrm{e}$ & $\mathrm{eV}$ \\
\hline 176 . & 38 e1u & 2.000 & -0.140966 & $\mathrm{H}=$ & $-3.836 \mathrm{e}$ & $\mathrm{eV}$ \\
\hline 175 . & $37 \mathrm{e} 1 \mathrm{u}$ & 2.000 & -0.140966 & $\mathrm{H}=$ & $-3.836 \mathrm{e}$ & $\mathrm{eV}$ \\
\hline 174 . & $19 \mathrm{au}$ & 2.000 & -0.157324 & $\mathrm{H}=$ & $-4.281 \mathrm{e}$ & $\mathrm{eV}$ \\
\hline 173. & $34 \mathrm{e} 2 \mathrm{u}$ & 2.000 & -0.162192 & $\mathrm{H}=$ & $-4.413 \mathrm{e}$ & $\mathrm{eV}$ \\
\hline 172 . & $33 \mathrm{e} 2 \mathrm{u}$ & 2.000 & -0.162192 & $\mathrm{H}=$ & $-4.413 \mathrm{e}$ & $\mathrm{eV}$ \\
\hline 171. & 32 e $2 u$ & 2.000 & -0.167073 & $\mathrm{H}=$ & $-4.546 \mathrm{e}$ & $\mathrm{eV}$ \\
\hline 170. & $31 \mathrm{e} 2 \mathrm{u}$ & 2.000 & -0.167073 & $\mathrm{H}=$ & $-4.546 \mathrm{e}$ & $\mathrm{eV}$ \\
\hline 169. & $34 \mathrm{e} 2 \mathrm{~g}$ & 2.000 & -0.174680 & $\mathrm{H}=$ & $-4.753 \mathrm{e}$ & $\mathrm{eV}$ \\
\hline 168. & 33 e $2 g$ & 2.000 & -0.174680 & $\mathrm{H}=$ & $-4.753 \mathrm{e}$ & $\mathrm{eV}$ \\
\hline 167. & 36 e $1 u$ & 2.000 & -0.175868 & $\mathrm{H}=$ & $-4.786 \mathrm{e}$ & $\mathrm{eV}$ \\
\hline 166. & 35 e1u & 2.000 & -0.175868 & $\mathrm{H}=$ & $-4.786 \mathrm{e}$ & $\mathrm{eV}$ \\
\hline 165. & $30 \mathrm{e} 2 \mathrm{u}$ & 2.000 & -0.250657 & $\mathrm{H}=$ & $-6.821 \mathrm{e}$ & $\mathrm{eV}$ \\
\hline 164 . & 29 e $2 u$ & 2.000 & -0.250657 & $\mathrm{H}=$ & $-6.821 \mathrm{e}$ & $\mathrm{eV}$ \\
\hline 163. & $31 \mathrm{e} 1 \mathrm{~g}$ & 2.000 & -0.250698 & $\mathrm{H}=$ & $-6.822 \mathrm{e}$ & $\mathrm{eV}$ \\
\hline 162 . & $32 \mathrm{e} 1 \mathrm{~g}$ & 2.000 & -0.250698 & $\mathrm{H}=$ & $-6.822 \mathrm{e}$ & $\mathrm{eV}$ \\
\hline 161. & $18 \mathrm{au}$ & 2.000 & -0.262188 & $\mathrm{H}=$ & $-7.135 \mathrm{e}$ & $\mathrm{eV}$ \\
\hline
\end{tabular}

\section{$2.7 \quad \mathrm{Lr}$}

Total energy $=-2573.0059921290 \mathrm{H}=-70015.0952542 \mathrm{eV}$
HOMO-LUMO Separation
$\begin{array}{clll}\text { HOMO: } & \text { 353. a } 20 \mathrm{ag} & -0.11093725 \mathrm{H}= & -3.01876 \mathrm{eV} \\ \text { LUMO: } & 354 . \mathrm{b} 20 \mathrm{ag} & -0.06848060 \mathrm{H}= & -1.86345 \mathrm{eV} \\ \text { Gap : } & & +0.04245665 \mathrm{H}= & +1.15530 \mathrm{eV}\end{array}$




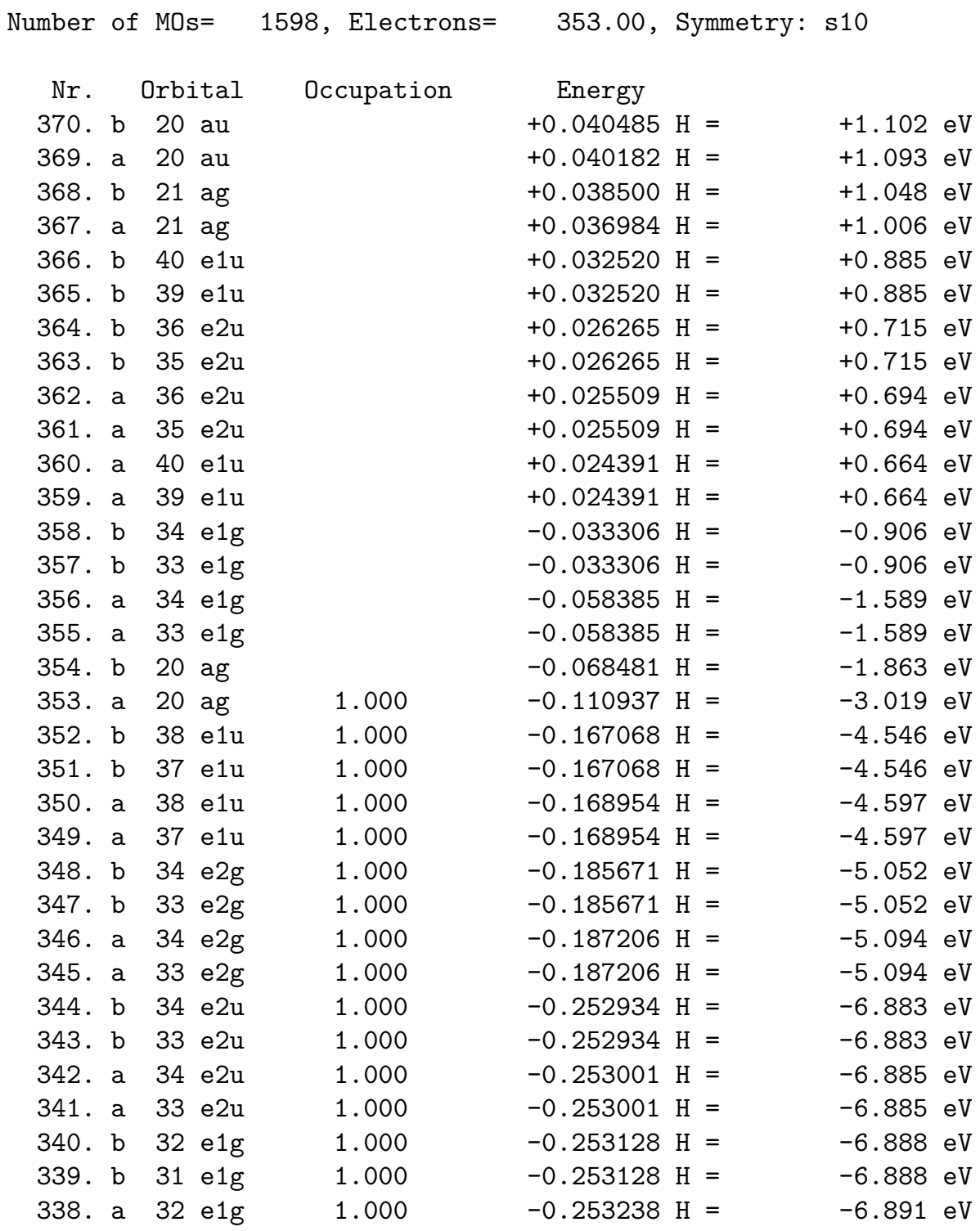

\section{Orbital contour plots and energies for $\operatorname{An}\left(\mathrm{Cp}^{\mathrm{iPr}}\right)_{2}$}

\section{$3.1 \quad$ Th}

Configuration Energy $(\mathrm{eV})$ 
$20 a_{g}(\mathrm{HOMO}) \quad-2.218$

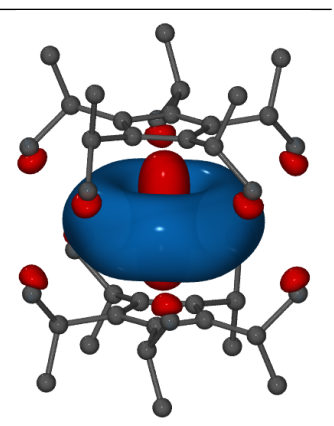

$33 e_{1 g}(\mathrm{LUMO})$

$-1.982$

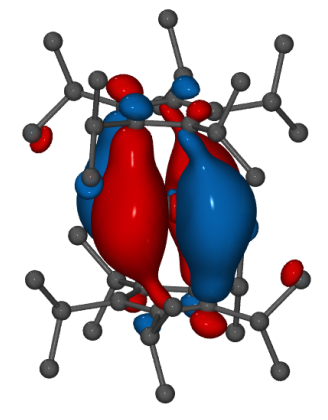

$34 e_{1 g}$

$-1.982$

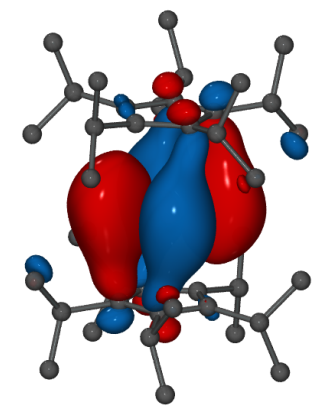

$31 e_{2 u}$

$-1.511$

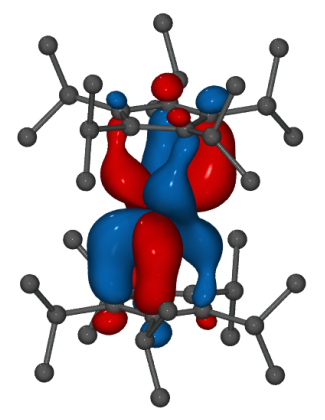


$32 e_{2 u}$

$33 e_{2 u}$

$34 e_{2 u}$

$19 a_{u}$
$-1.511$

$-1.283$

$-1.283$

$-1.029$
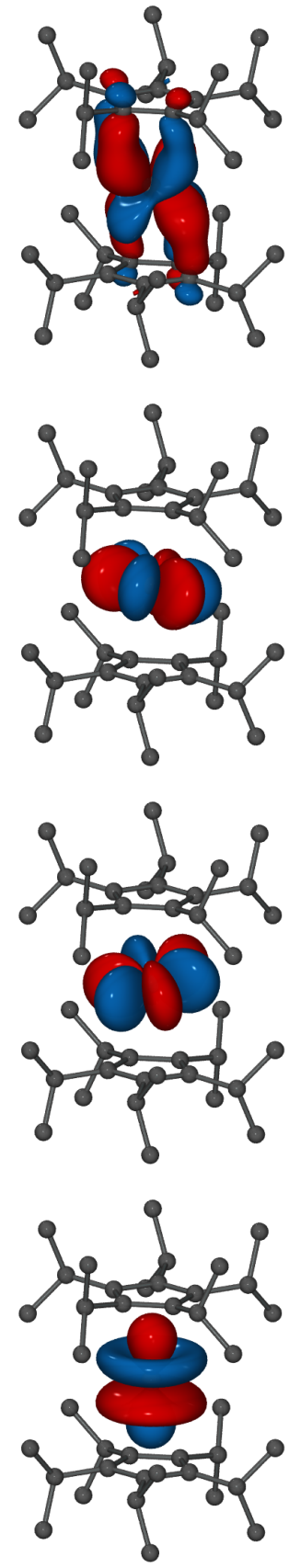
$37 e_{1 u}$

$38 e_{1 u}$

$-0.769$

$-0.769$

Table 1: Contour plots of selected orbitals computed by DFT using the TPSS functional.
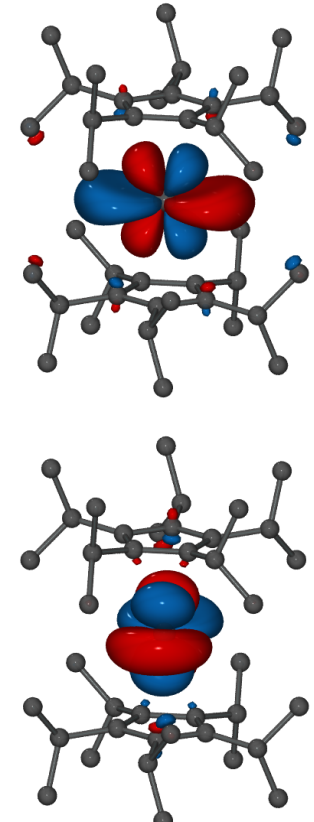
$3.2 \mathrm{U}$

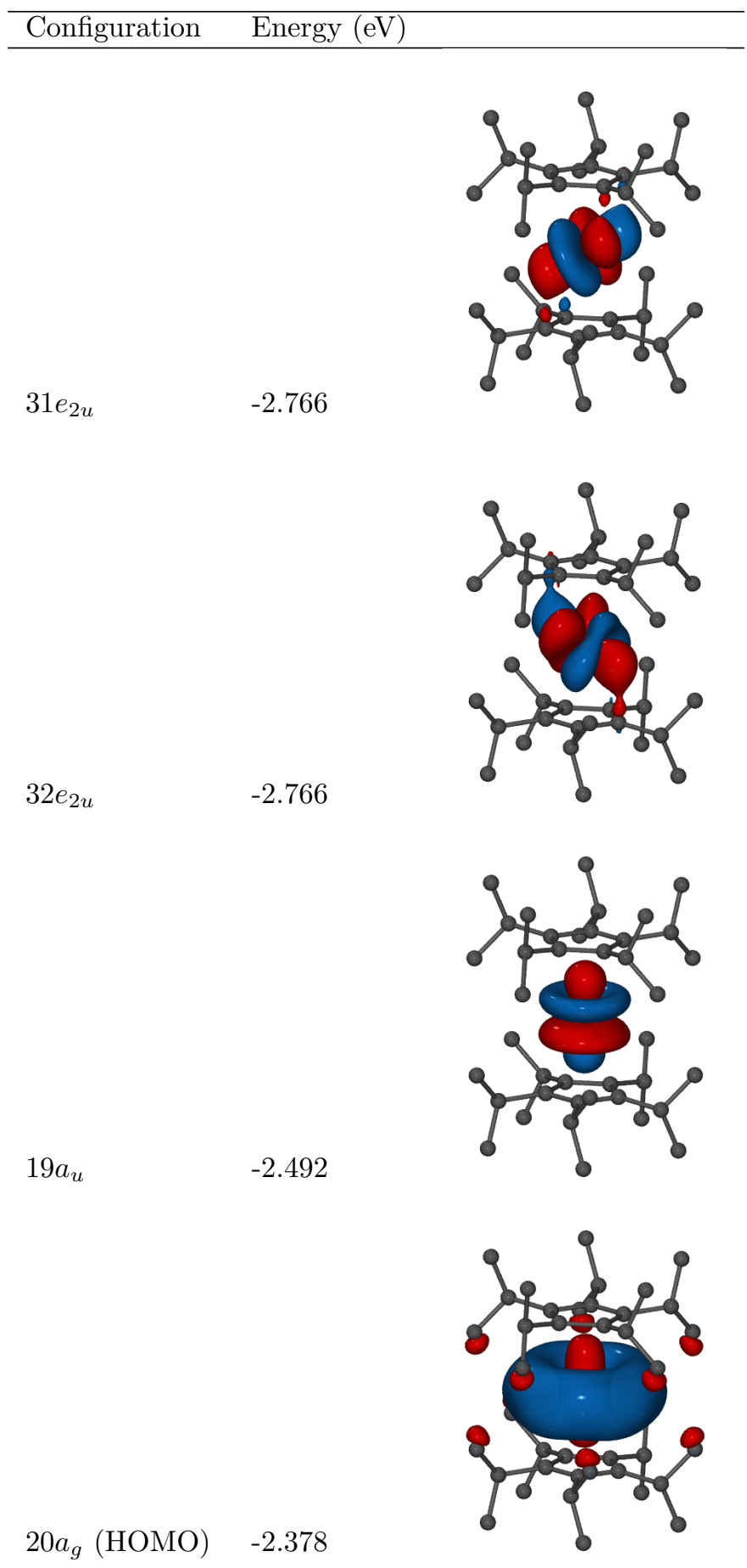


$33 e_{1 g}$ (LUMO)

$-1.948$

$34 e_{1 g}$

$33 e_{2 u}$

$34 e_{2 u}$

$-1.399$

$-1.948$

$-1.399$
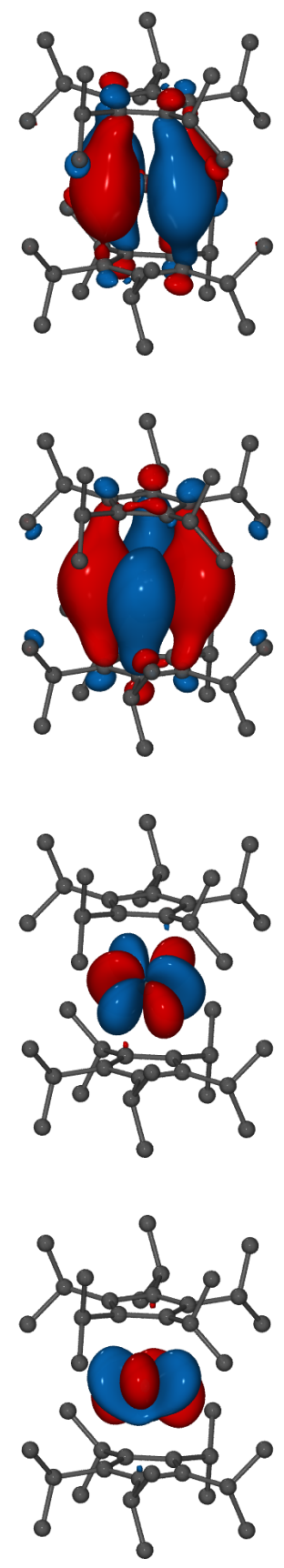
$37 e_{1 u}$

$38 e_{1 u}$ $-0.941$

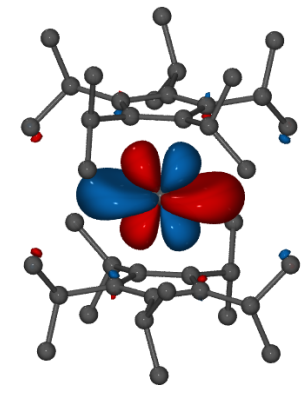

Table 2: Contour plots of selected orbitals computed by DFT using the TPSS functional. 


\section{3 $\mathrm{Pu}$}

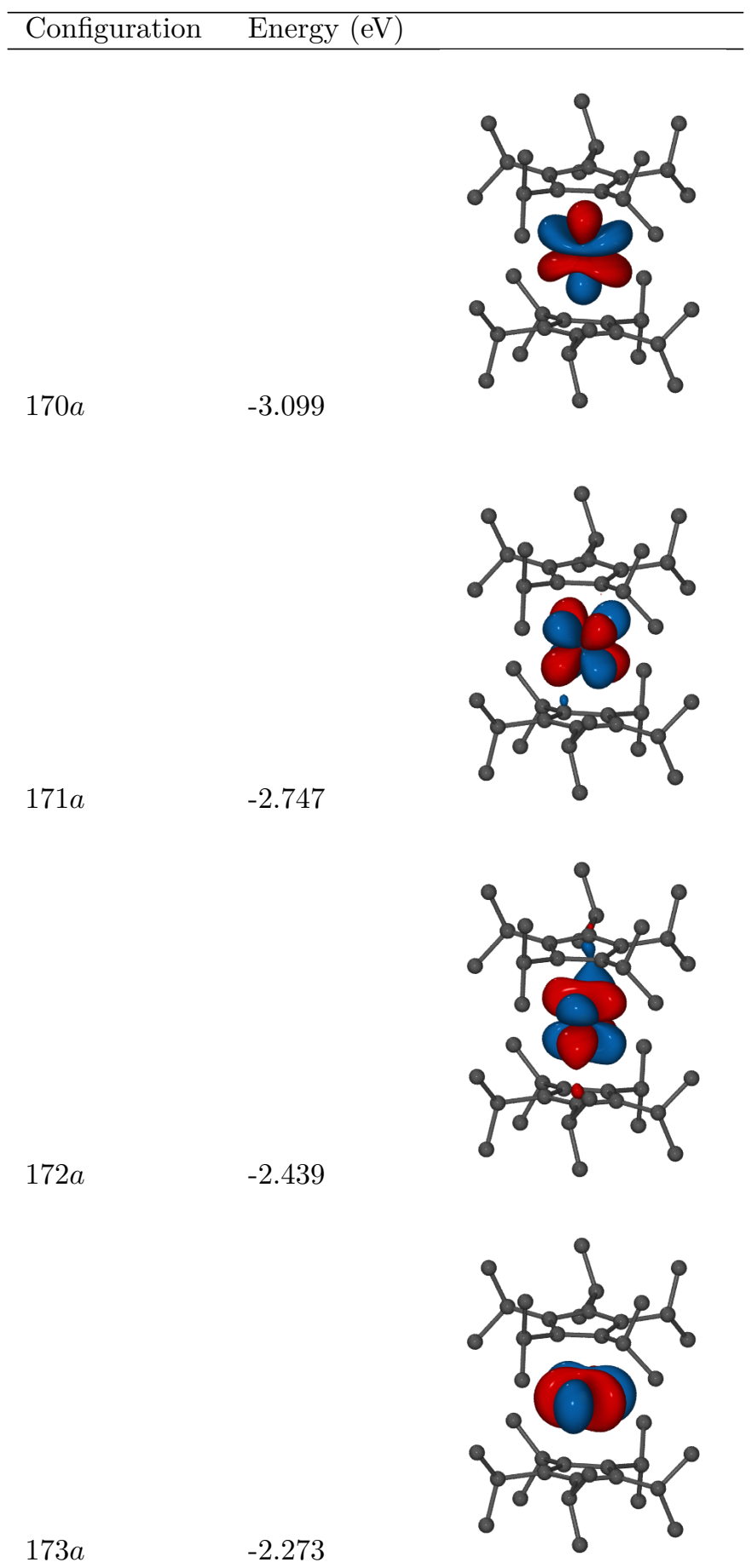


$174 a$

$-2.220$

175a (HOMO)

$-2.097$

176a (LUMO)

$-1.785$

$177 a$

$-1.722$
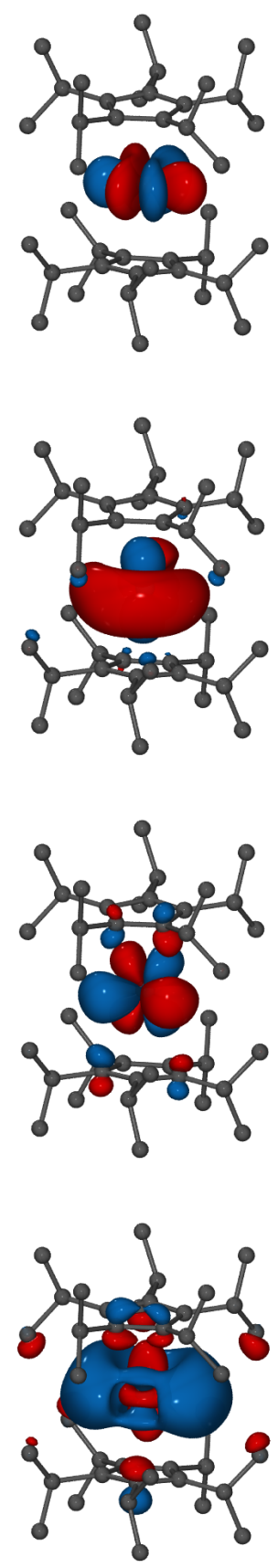
$178 a$

$179 a$

$-1.292$

$-1.224$

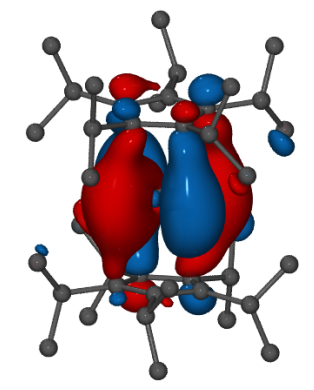

Table 3: Contour plots of selected orbitals computed by DFT using the TPSS functional. 
3.4 Am

Configuration Energy (eV)

$170 a$

$171 a$

$172 a$

$173 a$

$-2.821$

$-2.895$

$-2.822$
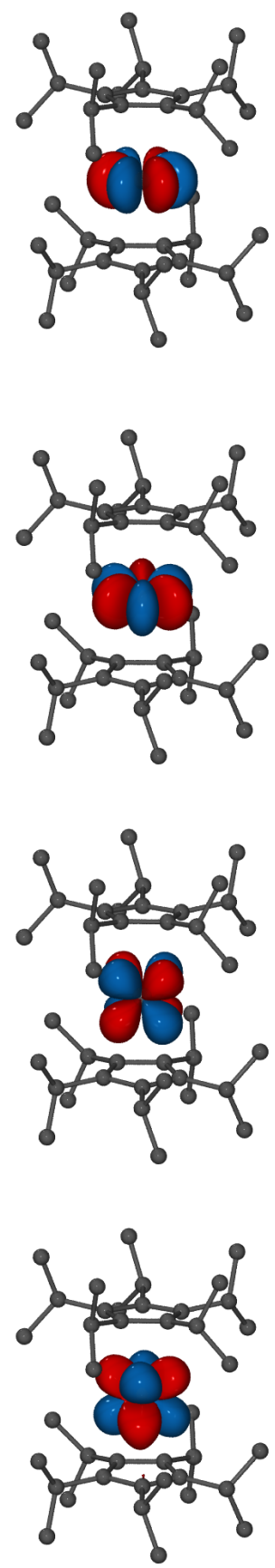
$174 a$

$175 a$

$176 a$

$177 a$
$-2.678$

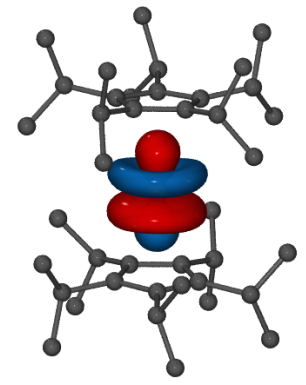

$-2.457$

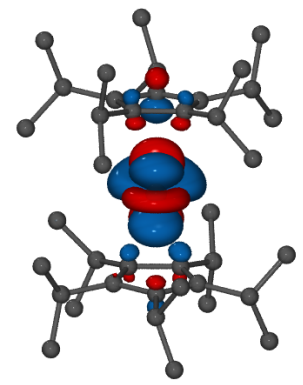

-2.408 (HOMO)
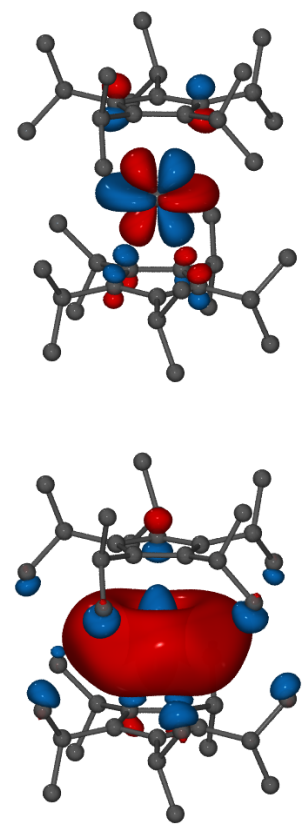
$178 a$

$179 a$

Table 4: Contour plots of selected orbitals computed by DFT using the TPSS functional.
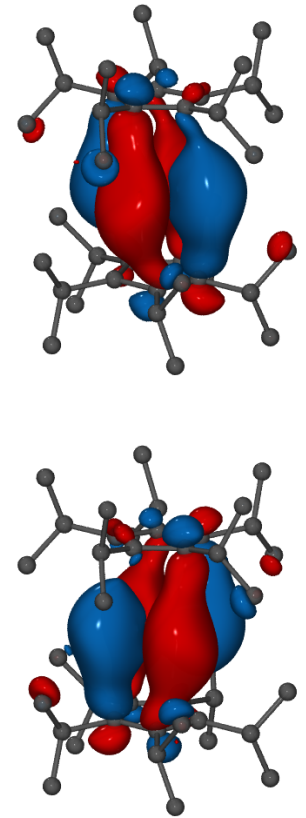


\section{$3.5 \quad \mathrm{Bk}$}

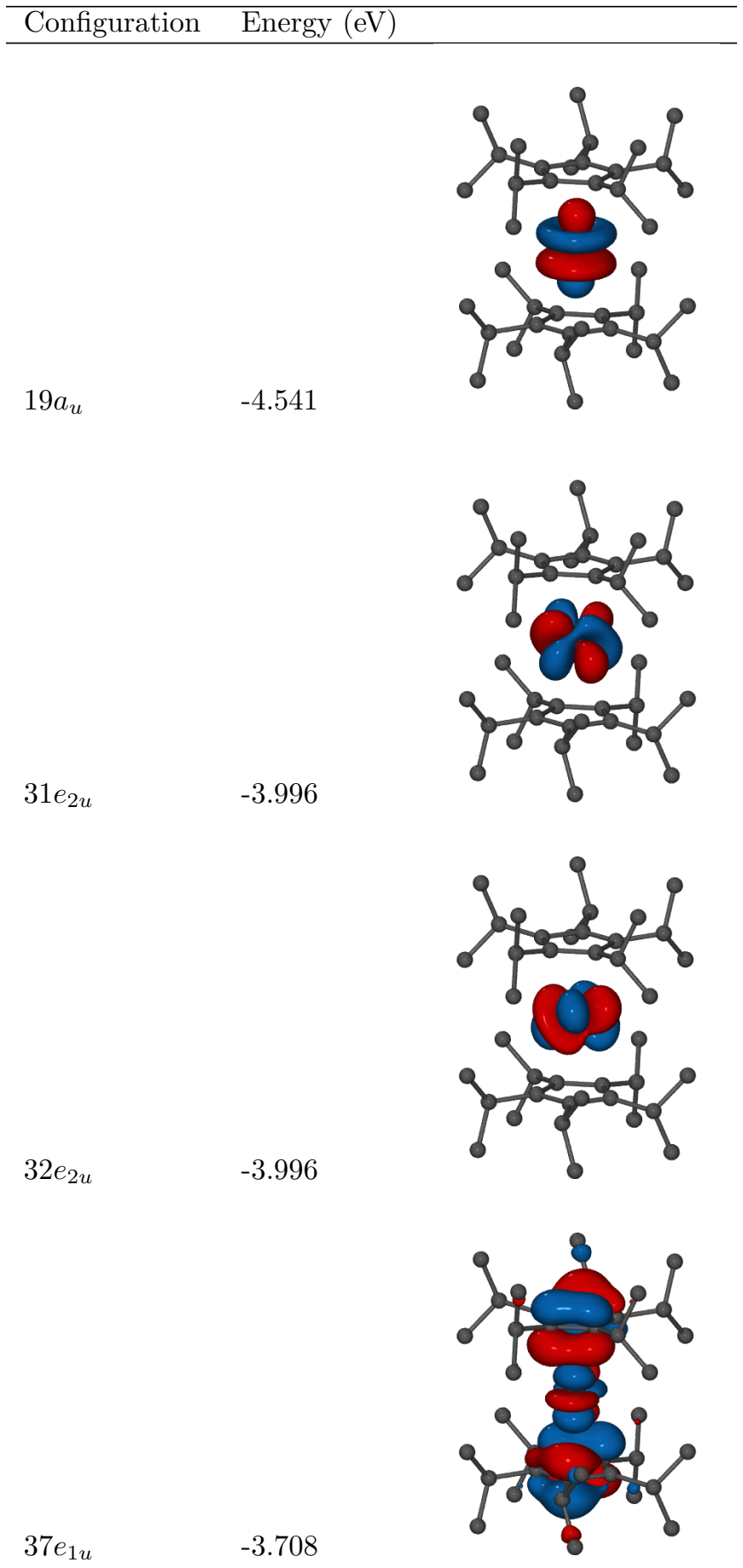


$38 e_{1 u}$

$33 e_{2 u}$

$34 e_{2 u}$

$-3.379$

$20 a_{g}(\mathrm{LUMO}) \quad-1.531$
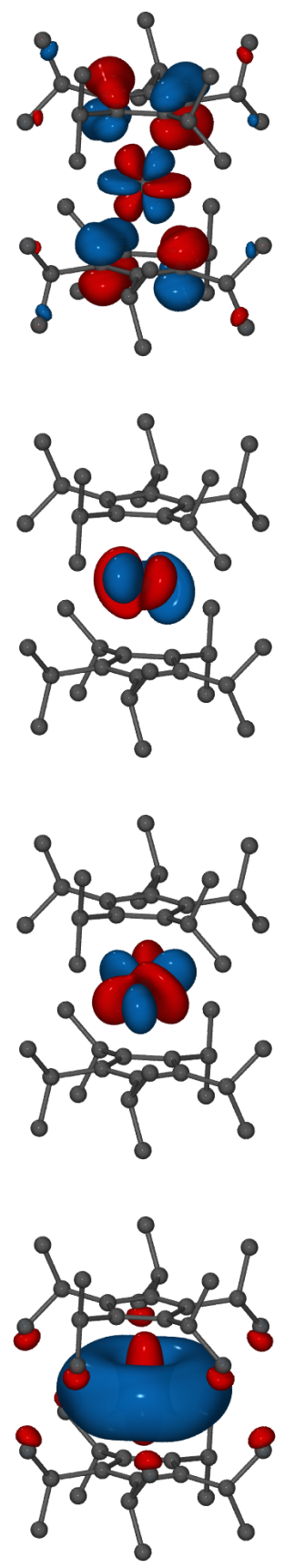
$33 e_{1 g}$

$34 e_{1 g}$ $-0.838$
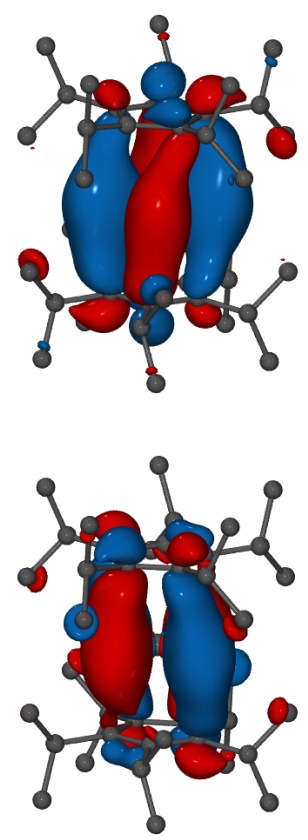

Table 5: Contour plots of selected orbitals computed by DFT using the TPSS functional. The contours of the degenerate pair of HOMO orbitals $31 e_{2 u}$ and $32 e_{2 u}$ are identical to the opposite spin $31 e_{2 u}$ and $32 e_{2 u}$ orbitals shown above, and are thus not included. 


\section{$3.6 \quad$ No}

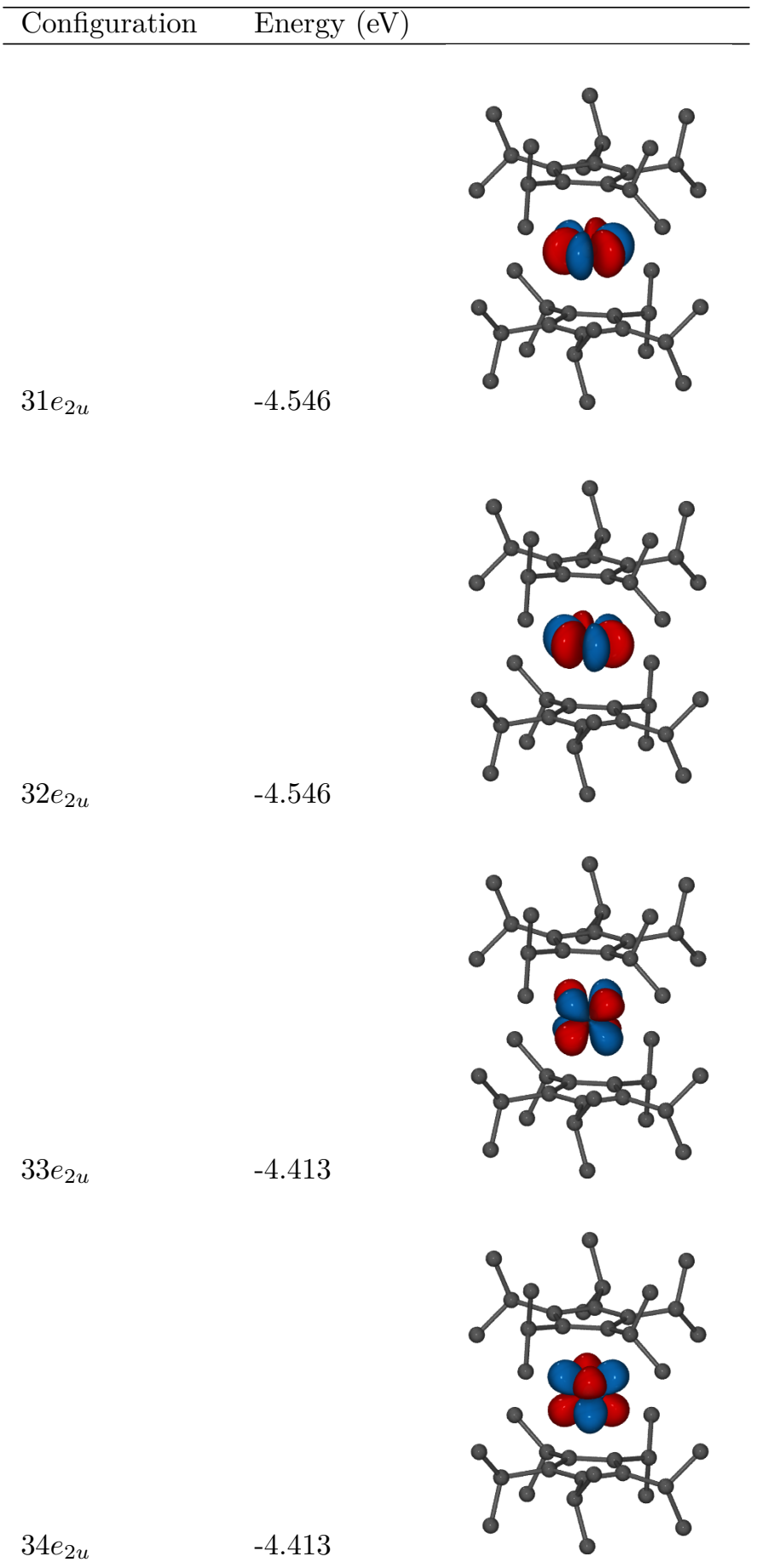


$19 a_{u}$

$37 e_{1 u}$

$-4.281$

$-3.836$

$38 e_{1 u}(\mathrm{HOMO})$

$-3.836$

$20 a_{g}$ (LUMO)

$-1.434$
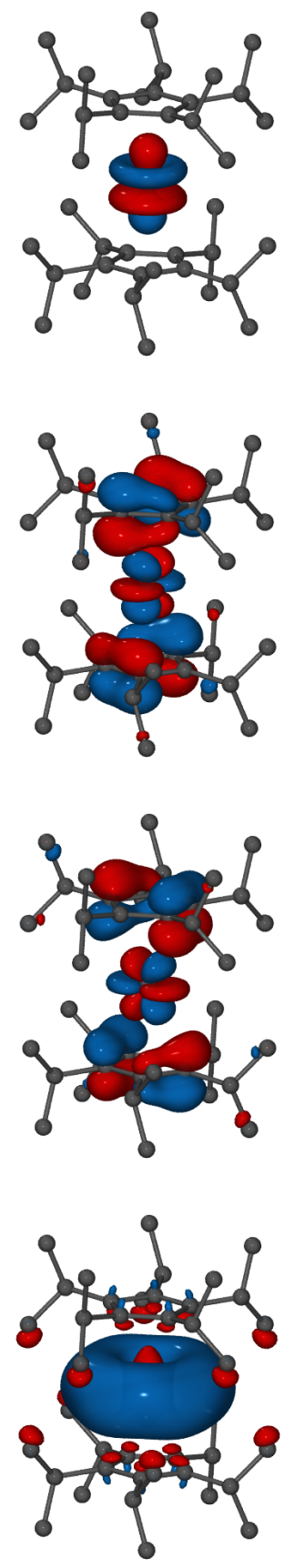
$33 e_{1 g}$

$34 e_{1 g}$

$-0.486$

Table 6: Contour plots of selected orbitals computed by DFT using the TPSS functional.
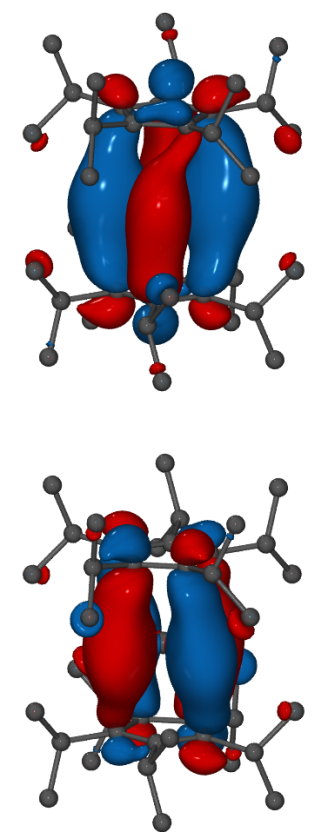
3.7 Lr

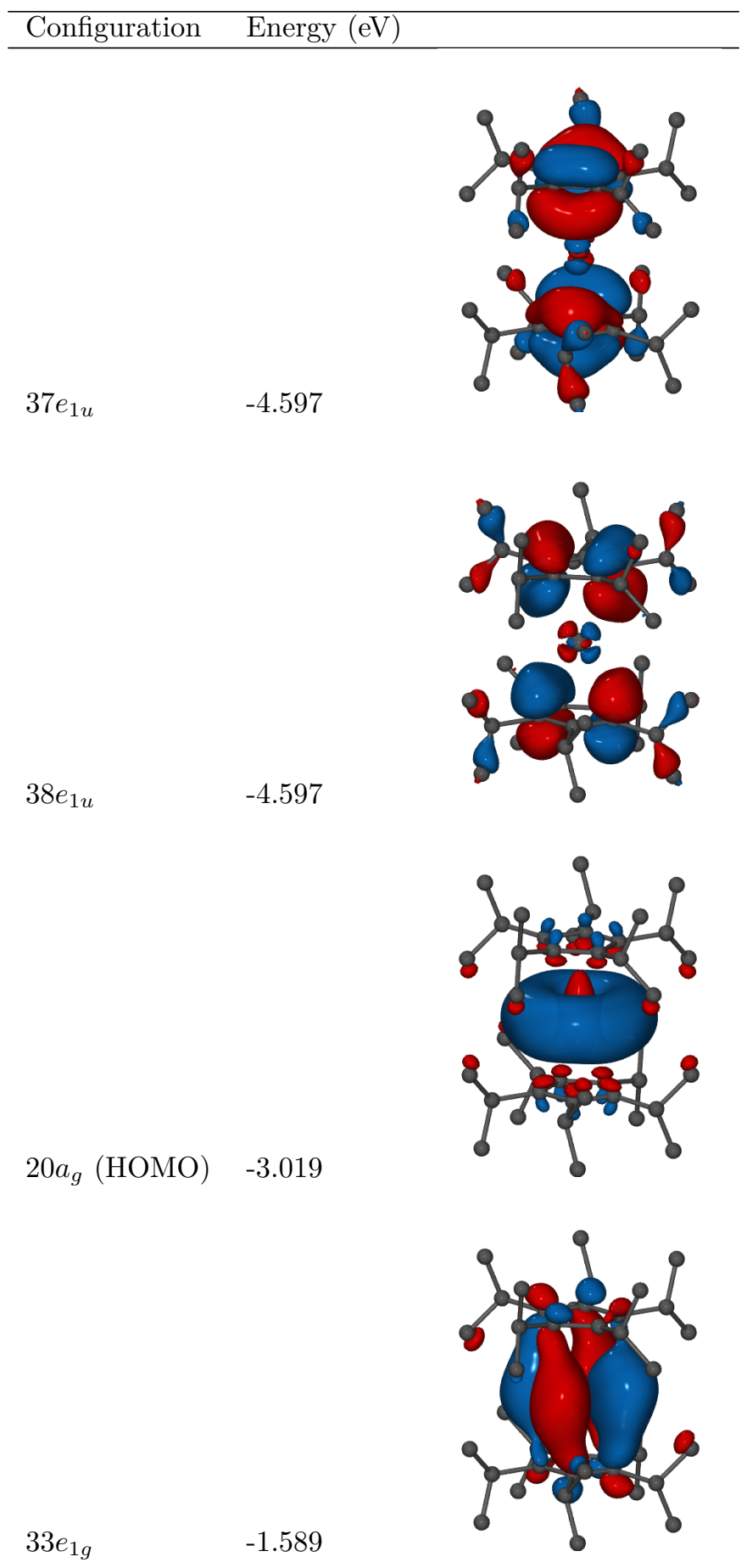


$34 e_{1 g}$ $-1.589$

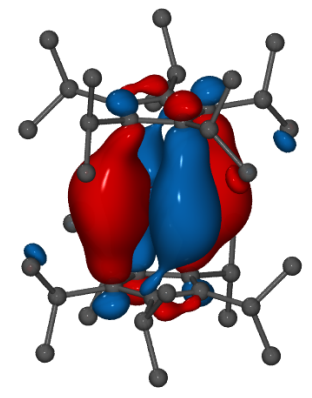

Table 7: Contour plots of selected orbitals computed by DFT using the TPSS functional.

\section{Tabulated Absorption Spectra Data for $\operatorname{An}\left(\mathrm{Cp}^{\mathrm{iPr} r_{5}}\right)_{2}$}

\subsection{Th}

\begin{tabular}{lllll}
\hline Wavelength $(\mathrm{nm})$ & Osc. Str. & Occ-virt trans. & Occ-virt energy $(\mathrm{eV})$ & Ex. type \\
\hline 933.9 & 0.023 & $20 a_{g} \rightarrow 19 a_{u}$ & $-2.22(-1.03)$ & $\mathrm{d} \rightarrow \mathrm{f}$ \\
404.0 & 0.097 & $20 a_{g} \rightarrow 40 e_{1 u}$ & $-2.22(0.77)$ & MLCT \\
383.2 & 0.051 & $20 a_{g} \rightarrow 20 a_{u}$ & $-2.22(1.00)$ & $\mathrm{d} \rightarrow \mathrm{f}$ \\
264.0 & 0.118 & $20 a_{g} \rightarrow 44 e_{1 u}$ & $-2.22(2.32)$ & MLCT \\
263.4 & 0.055 & $34 e_{2 g} \rightarrow 38 e_{1 u}$ & $-5.39(-0.77)$ & LMCT \\
\hline
\end{tabular}

Table 8: Characterization of selected excitations including excitation wavelength, predicted oscillator strength, terms for involved molecular orbitals, energies for involved molecular orbitals, and type of excitation. Results obtained with DFT using the TPSSh functional.

\section{$4.2 \quad \mathrm{U}$}

\begin{tabular}{lllll}
\hline Wavelength $(\mathrm{nm})$ & Osc. Str. & Occ-virt trans. & Occ-virt energy $(\mathrm{eV})$ & Ex. type \\
\hline 771.3 & 0.011 & $20 a_{g} \rightarrow 38 e_{1 u}$ & $-2.63(-0.23)$ & $\mathrm{d} \rightarrow \mathrm{f}$ \\
375.4 & 0.048 & $20 a_{g} \rightarrow 40 e_{1 u}$ & $-2.63(1.08)$ & MLCT \\
334.1 & 0.030 & $20 a_{g} \rightarrow 20 a_{u}$ & $-2.63(1.36)$ & MLCT \\
322.2 & 0.037 & $36 e_{1 u} \rightarrow 34 e_{1 g}$ & $-4.92(-0.61)$ & $\mathrm{f} \rightarrow \mathrm{d}$ \\
272.2 & 0.032 & $20 a_{g} \rightarrow 42 e_{1 u}$ & $-2.63(2.36)$ & MLCT \\
253.8 & 0.036 & $32 e_{2 u} \rightarrow 36 e_{1 g}$ & $-3.42(1.94)$ & MLCT \\
\hline
\end{tabular}

Table 9: Characterization of selected excitations including excitation wavelength, predicted oscillator strength, terms for involved molecular orbitals, energies for involved molecular orbitals, and type of excitation. Results obtained with DFT using the TPSSh functional. 


\section{3 $\mathrm{Pu}$}

\begin{tabular}{lllll}
\hline Wavelength $(\mathrm{nm})$ & Osc. Str. & Occ-virt trans. & Occ-virt energy $(\mathrm{eV})$ & Ex. type \\
\hline 759.9 & 0.012 & $171 a \rightarrow 177 a$ & $-3.39(-1.03)$ & $\mathrm{f} \rightarrow \mathrm{d}(45 \%)$ \\
759.9 & 0.012 & $171 a \rightarrow 179 a$ & $-3.39(-0.71)$ & $\mathrm{f} \rightarrow \mathrm{d}(40 \%)$ \\
391.1 & 0.015 & $175 a \rightarrow 181 a$ & $-2.48(1.08)$ & MLCT \\
\hline
\end{tabular}

Table 10: Characterization of selected excitations including excitation wavelength, predicted oscillator strength, terms for involved molecular orbitals, energies for involved molecular orbitals, and type of excitation. Results obtained with DFT using the TPSSh functional.

\subsection{Am}

\begin{tabular}{lllll}
\hline Wavelength $(\mathrm{nm})$ & Osc. Str. & Occ-virt trans. & Occ-virt energy $(\mathrm{eV})$ & Ex. type \\
\hline 653.3 & 0.017 & $172 a \rightarrow 179 a$ & $-3.46(-0.76)$ & $\mathrm{f} \rightarrow \mathrm{d}(27 \%)$ \\
653.3 & 0.017 & $173 a \rightarrow 179 a$ & $-3.45(-0.76)$ & $\mathrm{f} \rightarrow \mathrm{d}(26 \%)$ \\
653.3 & 0.017 & $173 a \rightarrow 179 a$ & $-3.46(-0.76)$ & $\mathrm{f} \rightarrow \mathrm{d}(23 \%)$ \\
653.3 & 0.017 & $172 a \rightarrow 178 a$ & $-3.46(-0.76)$ & $\mathrm{f} \rightarrow \mathrm{d}(22 \%)$ \\
379.5 & 0.007 & $167 a \rightarrow 177 a$ & $-5.19(-1.39)$ & LMCT \\
\hline
\end{tabular}

Table 11: Characterization of selected excitations including excitation wavelength, predicted oscillator strength, terms for involved molecular orbitals, energies for involved molecular orbitals, and type of excitation. Results obtained with DFT using the TPSSh functional.

\subsection{Bk}

\begin{tabular}{lllll}
\hline Wavelength $(\mathrm{nm})$ & Osc. Str. & Occ-virt trans. & Occ-virt energy $(\mathrm{eV})$ & Ex. type \\
\hline 1040.6 & 0.01353 & $32 e_{2 u} \rightarrow 34 e_{1 g}$ & $-2.27(-0.20)$ & $\mathrm{f} \rightarrow \mathrm{d}$ \\
472.2 & 0.011 & $34 e_{2 u} \rightarrow 34 e_{1 g}$ & $-4.13(-0.5)$ & $\mathrm{f} \rightarrow \mathrm{d}$ \\
399.2 & 0.016 & $38 e_{1 u} \rightarrow 34 e_{1 g}$ & $-4.23(-0.5)$ & $\mathrm{f} \rightarrow \mathrm{d}$ \\
364.6 & 0.0187 & $32 e_{2 u} \rightarrow 34 e_{1 g}$ & $-4.78(-0.5)$ & $\mathrm{f} \rightarrow \mathrm{d}$ \\
311.1 & 0.013 & $32 e_{2 u} \rightarrow 36 e_{1 g}$ & $-2.27(2.08)$ & MLCT \\
307.8 & 0.016 & $32 e_{2 u} \rightarrow 36 e_{1 g}$ & $-2.27(2.08)$ & MLCT $(44 \%)$ \\
307.8 & 0.016 & $36 e_{1 u} \rightarrow 34 e_{1 g}$ & $-4.73(-0.20)$ & LMCT \\
\hline
\end{tabular}

Table 12: Characterization of selected excitations including excitation wavelength, predicted oscillator strength, terms for involved molecular orbitals, energies for involved molecular orbitals, and type of excitation. Results obtained with DFT using the TPSSh functional.

\subsection{No}

\begin{tabular}{lllll}
\hline Wavelength $(\mathrm{nm})$ & Osc. Str. & Occ-virt trans. & Occ-virt energy $(\mathrm{eV})$ & Ex. type \\
\hline
\end{tabular}




\begin{tabular}{lllll}
\hline 316.6 & 0.061 & $38 e_{1 u} \rightarrow 34 e_{1 g}$ & $-4.43(-0.12)$ & $\mathrm{f} \rightarrow \mathrm{d}$ \\
265.3 & 0.028 & $34 e_{2 u} \rightarrow 34 e_{1 g}$ & $-5.40(-0.12)$ & $\mathrm{f} \rightarrow \mathrm{d}$ \\
254.2 & 0.041 & $36 e_{1 u} \rightarrow 34 e_{1 g}$ & $-5.56(-.12)$ & $\mathrm{f} \rightarrow \mathrm{d}$ \\
\hline
\end{tabular}

Table 13: Characterization of selected excitations including excitation wavelength, predicted oscillator strength, terms for involved molecular orbitals, energies for involved molecular orbitals, and type of excitation. Results obtained with DFT using the TPSSh functional.

\section{7 $\quad \mathrm{Lr}$}

\begin{tabular}{lllll}
\hline Wavelength $(\mathrm{nm})$ & Osc. Str. & Occ-virt trans. & Occ-virt energy $(\mathrm{eV})$ & Ex. type \\
\hline 307.9 & 0.137 & $38 e_{1 u} \rightarrow 34 e_{1 g}$ & $-4.93(-0.58)$ & LMCT $(51 \%)$ \\
307.9 & 0.137 & $20 a_{g} \rightarrow 40 e_{1 u}$ & $-3.28(0.95)$ & MLCT $(33 \%)$ \\
281.9 & 0.040 & $20 a_{g} \rightarrow 20 a_{u}$ & $-3.28(1.40)$ & MLCT \\
251.2 & 0.024 & $20 a_{g} \rightarrow 42 e_{1 u}$ & $-3.28(2.06)$ & MLCT \\
\hline
\end{tabular}

Table 14: Characterization of selected excitations including excitation wavelength, predicted oscillator strength, terms for involved molecular orbitals, energies for involved molecular orbitals, and type of excitation. Results obtained with DFT using the TPSSh functional.

\section{Optimized coordinates for solvated $\left[\mathbf{A n}\left(\mathbf{C p}^{\mathrm{iPr}}\right)_{2}\right]^{+}$cations}

\section{$5.1 \quad \mathrm{Th}$}

\$coord
-0.09767477362449
-0.50453169677927
0.34034386269913
0.63876568420582
-2.07884187282795
-4.90879225348588
-5.90573241603994
-5.65562496734204
-0.96124284936308
-2.31795944413135
-3.18992330834368
-4.54417346267785
1.75357453702460
3.76935645542310
3.66979693399703
3.83618847618754
2.29314282451415
4.82861017119315
5.73982816613648
1.92767995532476
4.78461644307524
6.18706866614392
5.93105683551316
0.08497398467866
0.62003053754651
1.99049900511062
1.99974360820359
$-2.37949136876708$
$-4.89418365307306$
$-5.05036201460493$
$-5.57416210232335$
$-2.05879725445930$
$-4.10341273446468$
$-5.18319064492254$
$-6.28886229672886$
0.62463125306202
1. 93251912636440
1.38278464536088

\begin{tabular}{|c|c|}
\hline 4.74592196908045 & c \\
\hline 4.68112523385721 & c \\
\hline 7.09640215570313 & C \\
\hline 2. 25989844954905 & c \\
\hline 4.75345301746183 & c \\
\hline 4.70020286366087 & c \\
\hline 7.07236267822444 & c \\
\hline 2.24195299393364 & c \\
\hline 4.62659389309672 & c \\
\hline 5.08979423679312 & c \\
\hline 7.87826601817401 & c \\
\hline 3. 31587793415122 & c \\
\hline 4.54189083431841 & c \\
\hline 4.93534342836357 & c \\
\hline 7.65553745923703 & c \\
\hline 3.00583307497698 & $C$ \\
\hline 4.62231420904349 & c \\
\hline 5.14630978459572 & C \\
\hline 7.87076109570871 & $c$ \\
\hline
\end{tabular}




\begin{tabular}{rr}
6.97566906809457 & 1.53827294958237 \\
-2.55634230695790 & 5.09254270860284 \\
-0.55777185945528 & 5.32446088422335 \\
-0.24691965948105 & 8.19349489001599 \\
2.40933688406193 & 6.13754760402181 \\
0.00079578774914 & 7.89632509163812 \\
2.72225643216461 & 5.90733612640225 \\
-0.01661916097899 & 4.90210698696288 \\
-5.85972638242181 & -1.22579226223611 \\
-5.40803013112724 & 0.91456161510163 \\
-7.99036092993923 & 2.15593294630787 \\
-5.11735186625584 & 3.91600186095652 \\
-7.69775996119305 & 1.82827222724655 \\
-5.17825877937818 & 4.02833449622151 \\
-4.72851130473904 & 1.12907780866197 \\
-0.88574384178129 & -6.37995219594742 \\
-1.62138564951746 & -4.63583889597295 \\
-3.92170385146162 & -6.96600215940080 \\
-4.72307220996724 & -3.67721584952446 \\
-4.86365748492678 & -7.64159937112573 \\
-6.33521431956986 & -4.66862061138331 \\
-4.17119337786632 & -5.00330447750729 \\
5.60230561901933 & -3.12962640560409 \\
3.43632850775300 & -3.66677034894583 \\
5.44349124974028 & -6.21261205314424 \\
2.08866380428807 & -6.53058419152832 \\
5.44996832196495 & -7.55177518561660 \\
4.08817175102941 & -5.58637375750461 \\
2.10065485071456 & -7.45207209489754 \\
4.42455020046897 & 3.97358710639091 \\
7.29924736294232 & 2.68544654081301 \\
4.18524451496285 & 1.65331001141532 \\
6.45401190624354 & -0.57039109239441 \\
8.65585816571523 & 2.63877446694923 \\
6.40996980672280 & 2.21801216876470 \\
7.55984972588612 & -0.46111528597126 \\
0.00027232572294 & 0.00018580591643 \\
0.09742187292066 & -1.92766942507743 \\
0.50411506624912 & -4.78462092070491 \\
-0.34084382915604 & -6.18748708793802 \\
-0.63924636631771 & -5.93062641499704 \\
2.07871965766579 & -0.08511437715191 \\
4.90865991866632 & -0.62036381610347 \\
5.90459932607383 & -1.99111040818045 \\
5.65649335854892 & -1.99978263726107 \\
0.96131672135950 & 2.37943464909834 \\
2.31827751165842 & 4.89402404382819 \\
3.19074382156061 & 5.05016693266164 \\
-7.54415210568769 & 5.57385866398186 \\
\hline
\end{tabular}

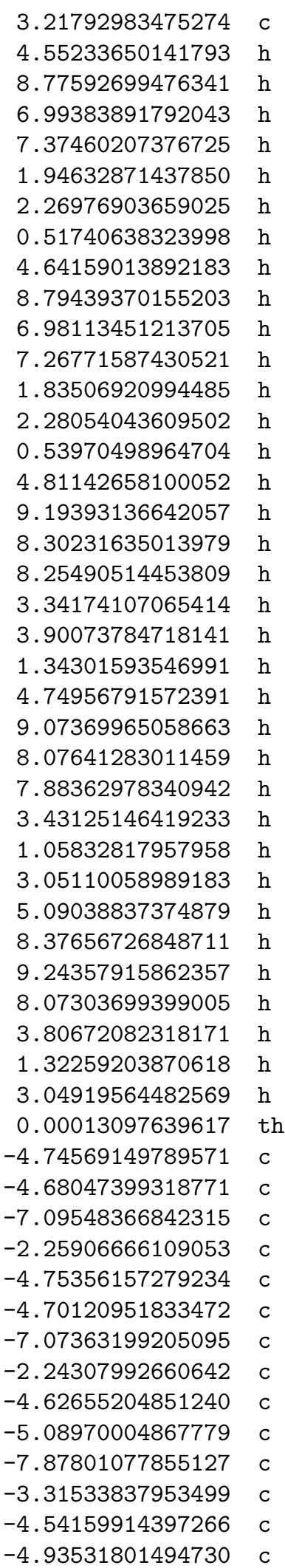




$$
\begin{aligned}
& -3.66932436188880 \\
& -3.83583431395230 \\
& -2.29329726879599 \\
& -4.82879849608753 \\
& -5.73976788128849 \\
& -6.97603671349445 \\
& \text { 2. } 55591072130073 \\
& 0.55714765331603 \\
& 0.24652693335605 \\
& -2.40985590579541 \\
& -0.00141410543268 \\
& -2.72273317817160 \\
& 0.01619656561659 \\
& 5.85970686312886 \\
& 5.40631883013654 \\
& 7.98925132386232 \\
& 5.11599724627556 \\
& 7.69872689731284 \\
& 5.17955814441581 \\
& 4.72963125529506 \\
& 0.88614303021338 \\
& \text { 1. } 62241136743270 \\
& \text { 3. } 92269101711408 \\
& 4.72389910110960 \\
& 4.86477007364309 \\
& \text { 6. } 33491578763954 \\
& \text { 4. } 17005139542178 \\
& -5.60219274713333 \\
& -3.43610458544535 \\
& -5.44281403808129 \\
& -2.08792434515448 \\
& -5.44918226696616 \\
& -4.08845645046225 \\
& -2.09998991561657 \\
& -4.42482322586475 \\
& -7.29917939848769 \\
& -4.18510473712286 \\
& -6.45392700330898 \\
& -8.65622004824660 \\
& -6.41065434845268 \\
& -7.56008783565699
\end{aligned}
$$
\$end

$$
5.18303629565997
$$

6.28950641686367

$-0.62444932063359$

$-1.93225881648174$

$-1.38269392305179$

$-1.53781371942664$

$-5.09262366029535$

$-5.32518081722713$

$-8.19386170104681$

$-6.13808966849438$

$-7.89590238615598$

$-5.90672701971926$

$-4.90152842907981$

1. 22541993039992

$-0.91528927082294$

$-2.15668444223025$

$-3.91657155709038$

$-1.82791430216780$

$-4.02849407122377$

$-1.12926509699993$

6.37992221153691

4. 63575619949214

6. 96577979879249

3. 67698007399315

7.64111247284335

4.66696222109004

5.00436870069681

3. 13010759019837

3. 66635651592328

6. 21273905394286

6.53009016970231

7.55276076220087

5.58751676061034

7.45224897231921

$-3.97333812118490$

$-2.68537234477614$

$-1.65338396688531$

0.57047268805728

$-2.63819934028855$

$-2.21755630633186$

0.46161897161732
$-7.65568754466976$ $-3.00618386781468 \quad c$ $-4.62193306975739 \mathrm{c}$ $-5.14596009938677 \mathrm{c}$ $-7.87053382423602 \quad \mathrm{c}$ $-3.21783963577896 \mathrm{c}$ $-4.55160864913155 \mathrm{~h}$ $-8.77523033422125 \mathrm{~h}$ $-6.99258917553374 \mathrm{~h}$ $-7.37358825949527 \mathrm{~h}$ $-1.94525481992158 \mathrm{~h}$ $-2.26893015786660 \mathrm{~h}$ $-0.51668309610563 \mathrm{~h}$ $-4.64320722964511 \mathrm{~h}$ $-8.79555990634627 \mathrm{~h}$ $-6.98315544274758 \mathrm{~h}$ $-7.26852827278302 \mathrm{~h}$ $-1.83685053349612 \mathrm{~h}$ $-2.28143204284253 \mathrm{~h}$ $-0.54065047214700 \mathrm{~h}$ $-4.81160405692392 \mathrm{~h}$ $-9.19395834056289 \mathrm{~h}$ $-8.30186699451398 \mathrm{~h}$ $-8.25445615769205 \mathrm{~h}$ $-3.34229601478340 \mathrm{~h}$ $-3.89891824719583 \mathrm{~h}$ $-1.34229989724069 \mathrm{~h}$ $-4.74951498164899 \mathrm{~h}$ $-9.07360147279172 \mathrm{~h}$ $-8.07673917800384 \mathrm{~h}$ $-7.88394303623188 \mathrm{~h}$ $-3.43225254352789 \mathrm{~h}$ $-1.05858581568477 \mathrm{~h}$ $-3.05128613809366 \mathrm{~h}$ $-5.08986751563841 \mathrm{~h}$ $-8.37631846894604 \mathrm{~h}$ $-9.24322840526691 \mathrm{~h}$ $-8.07302675956693 \mathrm{~h}$ $-3.80686296635732 \mathrm{~h}$ $-1.32241815040314 \mathrm{~h}$ $-3.04923581664648 \mathrm{~h}$

\section{$5.2 \quad \mathrm{U}$}

$\$$ coord
$-0.27046665941585$
$-0.67589389273323$
2.30621332171042
0.12858506588231
5. 14772773886712
6.02240830345349
6.85515825954717

4.61117954196626
4.98091139589456 


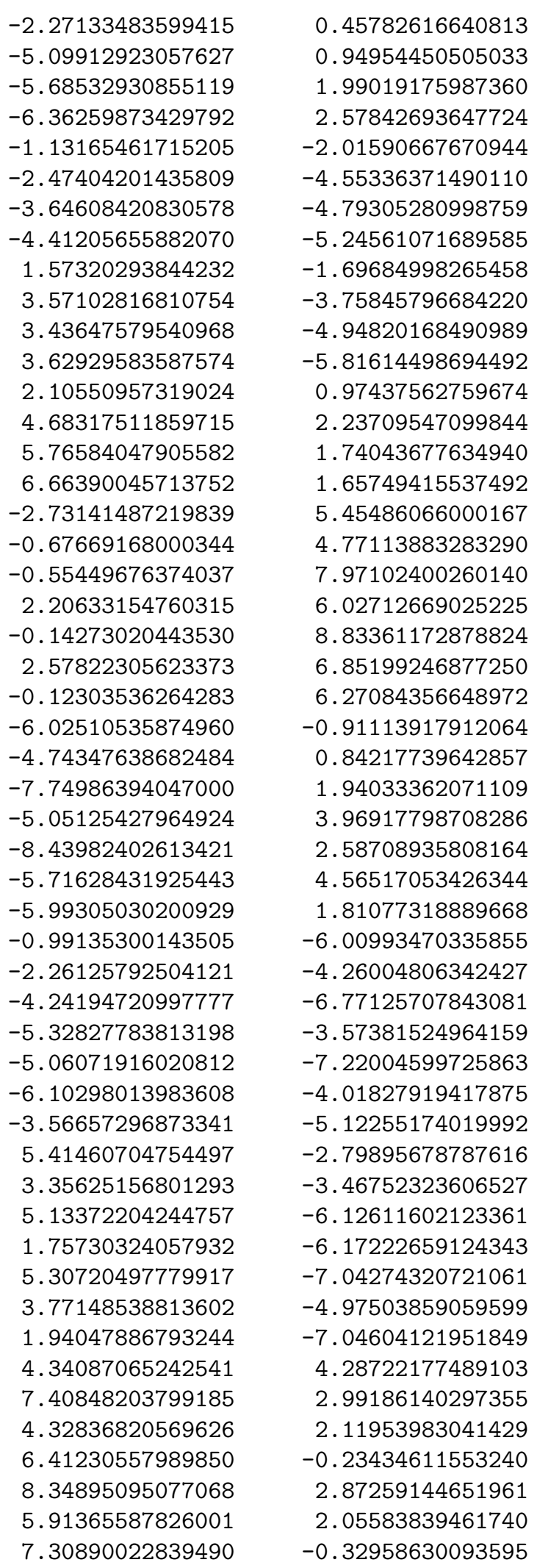

0.45782616640813

0.94954450505033

1. 99019175987360

2.57842693647724

$-2.01590667670944$

$-4.55336371490110$

$-4.79305280998759$

$-5.24561071689585$

$-1.69684998265458$

$-3.75845796684220$

$-4.94820168490989$

$-5.81614498694492$

0.97437562759674

2. 23709547099844

1. 74043677634940

1. 65749415537492

5.45486066000167

4.77113883283290

7.97102400260140

6.02712669025225

8. 83361172878824

6.85199246877250

6.27084356648972

$-0.91113917912064$

0.84217739642857

1. 94033362071109

3. 96917798708286

2. 58708935808164

4.56517053426344

1. 81077318889668

$-6.00993470335855$

$-4.26004806342427$

$-6.77125707843081$

$-3.57381524964159$

$-7.22004599725863$

$-4.01827919417875$

$-5.12255174019992$

$-2.79895678787616$

$-3.46752323606527$

$-6.12611602123361$

$-6.17222659124343$

$-7.04274320721061$

$-4.97503859059599$

$-7.04604121951849$

4.28722177489103

2. 99186140297355

2. 11953983041429

$-0.23434611553240$

2.87259144651961

2.05583839461740

$-0.32958630093595$

4.61224200849865

$4.98084906240924 \quad c$

7.64911305733829 c

$2.91894036098431 \quad \mathrm{c}$

$4.61326533540837 \quad \mathrm{c}$

$4.97808807897203 \mathrm{c}$

$7.64551464352405 \quad \mathrm{c}$

$2.91263929369675 \quad \mathrm{c}$

$4.61264705462713 \mathrm{c}$

$4.97680135360345 \quad \mathrm{c}$

$7.64375153218989 \quad \mathrm{c}$

$2.91152924214134 \quad \mathrm{c}$

4.61204101790462 c

$4.98162643722474 \quad \mathrm{c}$

$7.65159174135297 \quad \mathrm{C}$

$2.92196766356823 \quad \mathrm{c}$

$4.86548666934588 \mathrm{~h}$

$9.12010668768627 \mathrm{~h}$

$7.99841084990034 \mathrm{~h}$

$7.87748193094653 \mathrm{~h}$

$3.18105155607783 \mathrm{~h}$

$2.96433915014373 \mathrm{~h}$

$1.00962999172770 \mathrm{~h}$

$4.86950370194993 \mathrm{~h}$

$9.12019620102949 \mathrm{~h}$

$7.99388477076357 \mathrm{~h}$

$7.87067835218803 \mathrm{~h}$

$3.17274356378146 \mathrm{~h}$

$2.95427884654252 \mathrm{~h}$

$1.00645496135900 \mathrm{~h}$

$4.86491374735494 \mathrm{~h}$

$9.11768625905610 \mathrm{~h}$

$7.98511845627327 \mathrm{~h}$

$7.87028372425055 \mathrm{~h}$

$3.15675791585047 \mathrm{~h}$

$2.95169174892055 \mathrm{~h}$

$1.00056052370642 \mathrm{~h}$

$4.86302281649762 \mathrm{~h}$

$9.11688400253288 \mathrm{~h}$

$7.98360330956371 \mathrm{~h}$

$7.86740474878328 \mathrm{~h}$

$3.15546175759130 \mathrm{~h}$

$0.99943745571945 \mathrm{~h}$

$2.95276162339302 \mathrm{~h}$

$4.86822396269078 \mathrm{~h}$

$7.99676622116598 \mathrm{~h}$

$9.12104483909025 \mathrm{~h}$

$7.87552941506296 \mathrm{~h}$

$3.17318676329946 \mathrm{~h}$

$1.00824712102867 \mathrm{~h}$

$2.96199494747357 \mathrm{~h}$ 


\begin{tabular}{|c|c|c|}
\hline-0.00006313212889 & 0.00003313033032 & 0.00044436324250 \\
\hline 0.27035046571840 & -2.30624969421675 & -4.61030790143929 \\
\hline 0.67567051481420 & -5.14773569653268 & -4.98056374647805 \\
\hline-0.12895034266767 & -6.02174330326063 & -7.65198104733924 \\
\hline-0.48935785136448 & -6.85575735575167 & -2.92464588994169 \\
\hline 2.27132281653560 & -0.45794754453484 & -4.61136937839076 \\
\hline 5.09905237497540 & -0.94977546418326 & -4.98052203613828 \\
\hline 5.68454098864993 & -1.99049184618856 & -7.64892948068004 \\
\hline 6.36311195339468 & -2.57872142329349 & -2.91902141597185 \\
\hline 1.13175452905836 & 2.01585635966045 & -4.61249134615621 \\
\hline 2.47422462275924 & 4.55321143546078 & -4.97795162251167 \\
\hline 3.64620136301566 & 4.79206834199486 & -7.64549595832584 \\
\hline 4.41246364773983 & 5.24601931650538 & -2.91289595665921 \\
\hline-1.57312906727700 & 1.69692775374602 & -4.61192073083687 \\
\hline-3.57082303005618 & 3.75859083781693 & -4.97667611468076 \\
\hline-3.43575154288747 & 4.94786984839353 & -7.64381750593295 \\
\hline-3.62946214354589 & 5.81672962856268 & -2.91186433701449 \\
\hline-2.10557440318529 & -0.97428993162504 & -4.61125911897482 \\
\hline-4.68326932665800 & -2.23685443225142 & -4.98140245274790 \\
\hline-5.76539175001447 & -1.73970046499317 & -7.65150821276898 \\
\hline-6.66448851815176 & -1.65738997448059 & -2.92216738376754 \\
\hline 2.73118821662582 & -5.45494427454439 & -4.86521936495136 \\
\hline 0.67617206548567 & -4.77008496624112 & -9.11969623578526 \\
\hline 0.55412391572850 & -7.97026344454117 & -7.99881621215189 \\
\hline-2.20671660305610 & -6.02645149920221 & -7.87711823903168 \\
\hline 0.14262227192850 & -8.83409236878411 & -3.18181691272582 \\
\hline-2.57848382371702 & -6.85274880262900 & -2.96472989187582 \\
\hline 0.12256514557994 & -6.27192943881163 & 3856437389 \\
\hline 6.02509212662978 & 0.91089016088093 & -4.86937934801851 \\
\hline 4.74228065516327 & -0.84255368073627 & -9.11980312670672 \\
\hline 7.74898276338027 & -1.94066145152196 & -7.99427012906786 \\
\hline 5.05042250436574 & -3.96949252573135 & -7.87019741399868 \\
\hline 8.44026856630872 & -2.58720228817292 & -3.17345098616291 \\
\hline 5.71696785302964 & -4.56552835336263 & -2.95444917577216 \\
\hline 5.99396599692021 & -1.81128950999990 & -1.00638487177608 \\
\hline 0.99158579403476 & 6.00985756309098 & -4.86508397354257 \\
\hline 2.26139063895212 & 4.25857100085058 & -9.11749691181182 \\
\hline 4.24210437501965 & 6.77014994079726 & -7.98575117587292 \\
\hline 5.32839331254798 & 3.57275539999078 & -7.86982792680357 \\
\hline 5.06096389953435 & 7.22044209865815 & -3.15759796422941 \\
\hline 6.10347266463033 & 4.01879641633457 & -2.95205228315514 \\
\hline 3.56731932450174 & 5.12326235751052 & -1.00066873405440 \\
\hline-5.41445246711461 & 2.79917068516900 & -4.86299914214750 \\
\hline-3.35539089322115 & 3.46695067668006 & -9.11669111270711 \\
\hline-5.13283781659767 & 6.12588405611985 & -7.98412909048984 \\
\hline-1.75644448026584 & 6.17172006841499 & -7.86738565363880 \\
\hline-5.30734160706600 & 7.04325112454849 & -3.15644714008938 \\
\hline-3.77194081017058 & 4.97604872558976 & -0.99962392841515 \\
\hline-1.94068312844053 & 7.04668503165278 & -2.95317040638062 \\
\hline-4.34110705003418 & -4.28701615733480 & -4.86818058055891 \\
\hline-7.40821362951319 & -2.99075804854261 & -7.99715731046619 \\
\hline
\end{tabular}




$$
\begin{aligned}
& -4.32776057680575 \\
& -6.41152314609739 \\
& -8.34947272809705 \\
& -5.91475877643255 \\
& -7.30951709320087
\end{aligned}
$$
\$end

\section{$5.3 \mathrm{Pu}$}

\$coord

$$
\begin{array}{r}
-0.26919165882949 \\
-0.67461653352987 \\
0.12117224773485 \\
0.50218879241006 \\
-2.27037920803847 \\
-5.09719740852532 \\
-5.68711650761019 \\
-6.35721314759375 \\
-1.13465313945677 \\
-2.47736432059449 \\
-3.64645812215247 \\
-4.41803927856411 \\
1.56819700023270 \\
3.56595525414351 \\
3.45640143074764 \\
3.59704888218081 \\
2.10324251881520 \\
4.68034062942759 \\
5.77026737020909 \\
6.65588295996715 \\
-2.72925028614695 \\
-0.70041874918789 \\
-0.54927716983724 \\
2.19769633461337 \\
-0.18802949336103 \\
2.58834039585578 \\
-0.03923250848662 \\
-6.02039205528032 \\
-4.75632520544052 \\
-7.75287933486151 \\
-5.04504620753302 \\
-8.43684851966305 \\
-5.74636123701283 \\
-5.94332390723055 \\
-0.99544262101196 \\
-2.25686671248553 \\
-4.25511989078455 \\
-5.32045839534728 \\
-5.04244029917899 \\
-6.12129571970274
\end{array}
$$

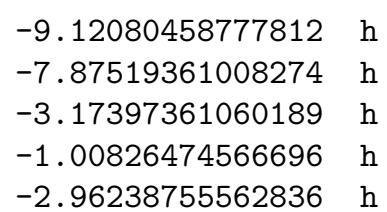

$\mathrm{h}$

$-2.87246563012769$

$-2.05574081853756$

0.32968441451293

$-2.96238755562836$
2. 29877513529919

5.14148024668667

6.04447492941448

6.82299791700522

0.45507422082047

0.94665953738522

1. 98996333274129

2.57362843594769

$-2.01802458786947$

$-4.55386400337750$

$-4.80314979802834$

$-5.23163322705999$

$-1.70254635552580$

$-3.76401415030075$

$-4.94586249608165$

$-5.82829107262424$

0.96587263961096

2. 23096031226967

1. 75791123861306

1. 63094374088403

5.44585176842190

4.81990302326973

8.00235800907232

6.03732663346880

8.78917075625822

6.87561789183284

6.16745008321729

$-0.91482070373024$

0.83872960902000

1. 94972272125478

3. 96618803671651

2. 54345570846498

4.57029583219185

1.83120542579349

$-6.00960937594686$

$-4.29020165862478$

$-6.77940680555099$

$-3.57475404450218$

$-7.21674346186975$

$-4.02411864097569$
$4.58906782080116 \quad \mathrm{C}$

$4.92284101164238 \quad \mathrm{c}$

$7.58713890915252 \quad \mathrm{c}$

$2.85180681458564 \quad \mathrm{c}$

$4.59829271713439 \quad \mathrm{c}$

$4.95383719215852 \quad \mathrm{c}$

$7.62045443633021 \quad \mathrm{c}$

$2.88837732622461 \quad \mathrm{C}$

$4.59989967691010 \quad \mathrm{C}$

$4.95107073222403 \mathrm{C}$

$7.61908347801157 \quad \mathrm{c}$

$2.88349282372115 \quad \mathrm{c}$

$4.59093827133551 \quad \mathrm{c}$

$4.93319500250829 \quad \mathrm{c}$

$7.60505250753307 \quad \mathrm{C}$

$2.87396077781409 \quad \mathrm{c}$

$4.58437637662615 \quad \mathrm{c}$

$4.92561867571344 \quad \mathrm{c}$

7.59694899827459 C

$2.86702257086637 \quad \mathrm{c}$

$4.79341521183968 \mathrm{~h}$

$9.06836072274159 \mathrm{~h}$

$7.90280134725031 \mathrm{~h}$

$7.82167227963083 \mathrm{~h}$

$3.03833563458563 \mathrm{~h}$

$2.94823340679550 \mathrm{~h}$

$0.94044742062603 \mathrm{~h}$

$4.84079754223689 \mathrm{~h}$

$9.09600398208881 \mathrm{~h}$

$7.95738573335985 \mathrm{~h}$

$7.84204523233636 \mathrm{~h}$

$3.11567879926972 \mathrm{~h}$

$2.95197669723445 \mathrm{~h}$

$0.97685144899791 \mathrm{~h}$

$4.82820818320350 \mathrm{~h}$

$9.09388276924976 \mathrm{~h}$

$7.94522008833168 \mathrm{~h}$

$7.85424080764614 \mathrm{~h}$

$3.09945129295976 \mathrm{~h}$

$2.95301967793009 \mathrm{~h}$ 


\begin{tabular}{|c|c|}
\hline-3.58208048303552 & -5.06124605836231 \\
\hline 5.40814885737818 & -2.80584265636177 \\
\hline 3.41058204392385 & -3.46285738839251 \\
\hline 5.14659676009568 & -6.13941721772666 \\
\hline 1.76900477638012 & -6.15352045986452 \\
\hline 5.30456957165076 & -7.02082414749798 \\
\hline 3.66027061848750 & -4.99058715329713 \\
\hline 1.93378187242469 & -7.08854316948931 \\
\hline 4.33535182122147 & 4.27884723511140 \\
\hline 7.42154517973737 & 3.00329757493466 \\
\hline 4.34250257983288 & 2.16073447066992 \\
\hline 6.40637385421982 & -0.21800184804682 \\
\hline 8.31666411012124 & 2.88647109371398 \\
\hline 5.88172498436945 & 1.95628048425793 \\
\hline 7.34181156463208 & -0.34008172151819 \\
\hline-0.01310488909926 & -0.01017563019197 \\
\hline 0.27026201692047 & -2.30148621886139 \\
\hline 0.67550915424098 & -5.14205311240770 \\
\hline-0.10925052215142 & -6.02187164669940 \\
\hline-0.50901736978018 & -6.84033616767948 \\
\hline 2.27135270256961 & -0.45748181163695 \\
\hline 5.10013782283789 & -0.94844613959395 \\
\hline 5.70890540794675 & -1.96786534999688 \\
\hline 6.34196403479449 & -2.59938798738765 \\
\hline 1.13555651704816 & 2.01602722286803 \\
\hline 2.47452751271971 & 4.55487720824254 \\
\hline 3.62790137807044 & 4.80963673318736 \\
\hline 4.43102373954661 & 5.23787184327664 \\
\hline-1.56771981792842 & 1.69932214405184 \\
\hline-3.56755206846712 & 3.75855536591041 \\
\hline-3.44482569621303 & 4.95760978039093 \\
\hline-3.62175769628838 & 5.80976133167863 \\
\hline-2.10188295981885 & -0.96874865901703 \\
\hline-4.67783221410601 & -2.23127269809149 \\
\hline-5.76624646257317 & -1.73558186527475 \\
\hline-6.65247452150057 & -1.65043590166402 \\
\hline 2.72942275275304 & -5.44917999515357 \\
\hline 0.72276649301636 & -4.78670180763144 \\
\hline 0.55899440291936 & -7.97840454581585 \\
\hline-2.18439559670132 & -6.00851112944363 \\
\hline 0.15848951741154 & -8.81098703429659 \\
\hline-2.59618589026808 & -6.87042912106269 \\
\hline 0.04940297514991 & -6.22016055957369 \\
\hline 6.02422247169023 & 0.91107495758856 \\
\hline 4.79290997710641 & -0.80145685519418 \\
\hline 7.77744747606435 & -1.92966444799454 \\
\hline 5.06377344571221 & -3.94043311425732 \\
\hline 8.42413886965986 & -2.55855468020890 \\
\hline 5.73985020536284 & -4.59731158660048 \\
\hline 5.90183975631709 & -1.88843832546975 \\
\hline 0.98976965162555 & 6.00700602294776 \\
\hline
\end{tabular}

\begin{tabular}{|c|c|}
\hline 0.97304034631137 & \\
\hline 4.79530774 & \\
\hline 9.07725897520438 & \\
\hline 7.92326170825207 & \\
\hline 7.85249096581332 & \\
\hline 3.07228096014333 & \\
\hline 862518480 & \\
\hline 2.97257632632873 & \\
\hline 4.79187521932910 & \\
\hline 7.920 & \\
\hline 9.06937993264255 & \\
\hline 7.83754110541430 & \\
\hline 3.07383686 & \\
\hline 18486 & \\
\hline 2.96016388 & \\
\hline 0.00337562317735 & \\
\hline 2608 & \\
\hline-4.9 & \\
\hline-7.61 & \\
\hline-2.89145824241634 & \\
\hline-4.57843698513565 & \\
\hline-4.91792135967194 & \\
\hline-7.58969664 & \\
\hline-2.86019432373169 & \\
\hline-4.57536147542565 & \\
\hline-4.92203166464216 & \\
\hline-7.59665933 & \\
\hline-2.87 & \\
\hline-4.58742307 & \\
\hline-4.93234235667940 & \\
\hline-7.59589963367722 & $c$ \\
\hline-2.86091919 & \\
\hline-4.59818277 & \\
\hline-4.95683596102446 & \\
\hline-7.62460485 & c \\
\hline-2.89178518159103 & \\
\hline-4.81035658853692 & $\mathrm{~h}$ \\
\hline-9.08658288 & \\
\hline-7.94837134621471 & \\
\hline-7.86592106099181 & \\
\hline-3.11171403087951 & \\
\hline-2.97702792078802 & \\
\hline-0.97329029543583 & \\
\hline-4.77986812563898 & \\
\hline-9.06241320567127 & \\
\hline-7.90929433429026 & \\
\hline-7.83421233055104 & \\
\hline-3.06124223626740 & \\
\hline-2.96048617135282 & \\
\hline-0.94291287750306 & \\
\hline 170660100 & \\
\hline
\end{tabular}




\begin{abstract}
2. 23403882517389
4. 22847285646600

5. 30518797542427

5.02356277141083

6. 15195759514728

3. 62613887041814

$-5.40736026635270$

$-3.37337916259218$

$-5.14270241403579$

$-1.76645615101514$

$-5.33570714842353$

$-3.68796467486968$

$-1.96670946670819$

$-4.33262502256493$

$-7.41530110308505$

$-4.33649142173877$

$-6.40527755248649$

$-8.31922094756660$

$-5.88111495017163$

$-7.32931547934744$
\end{abstract}

\$end

\section{$5.4 \quad \mathrm{Am}$}

\$coord

0.06971065031865

0.11279663804809

1.10536055854992

1.44789709437169

$-2.17829620089471$

$-4.90342700575064$

$-5.33228361450233$

$-5.93458013821872$

$-1.42974398962852$

$-3.15274044901953$

$-4.35441958068229$

$-5.18572858903402$

1. 27286647216534

2. 94692895820918

2. 63423716078143

2.74907359909958

2. 21200962843106

4.95306407305243

5.97295903878923

6.83506650372305

$-1.87900471569592$

0.14900777893661

0.73498780352501

3. 16428688549955

1. 13869564412780
4.29130795369649

6.78791958415811

3. 58668120681956

7. 23385422556120

4.05779484334640

5.03624109821328

2.79336593868587

3.48430733546912

6.13821178083922

6.18203031351183

6.99290835736457

4.96161866342205

7.08202360405955

$-4.28028222362282$

$-2.98063216574722$

$-2.12399413051398$

0.24113792377630

$-2.89522393113433$

$-2.00414345730295$

0.32482101873714
$-9.06536509502753 \mathrm{~h}$

$-7.92558478800014 \mathrm{~h}$

$-7.83768863976352 \mathrm{~h}$

$-3.07657263357128 \mathrm{~h}$

$-2.97553833216852 \mathrm{~h}$

$-0.95095782880885 \mathrm{~h}$

$-4.81365308027376 \mathrm{~h}$

$-9.07682100104500 \mathrm{~h}$

$-7.92127256132820 \mathrm{~h}$

$-7.82089950103713 \mathrm{~h}$

$-3.06053554004465 \mathrm{~h}$

$-0.94949481151836 \mathrm{~h}$

$-2.94488009806283 \mathrm{~h}$

$-4.84224438535647 \mathrm{~h}$

$-7.96053800050636 \mathrm{~h}$

$-9.09903600578662 \mathrm{~h}$

$-7.84958370973961 \mathrm{~h}$

$-3.11526442605153 \mathrm{~h}$

$-0.97994650067568 \mathrm{~h}$

$-2.95961067164210 \mathrm{~h}$
2.21995563622066

5.08935641936380

5.83075780692313

6.64541521648306

0.71331832094456

1. 61511491844702

2. 68096542971643

3. 45791366593929

$-1.94301327509466$

$-4.23969541780078$

$-4.30204841976166$

$-4.66170710153821$

$-2.05663867265184$

$-4.38944980000568$

$-5.50561363877231$

$-6.49287537845586$

0.52018670181718

1. 37840303121679

0.73494964739618

0.51475639645375

5.69426195330206

4.72913303565322

7.86228626662330

5.52517933806136

8. 68991213367968
$4.72484805307967 \quad \mathrm{c}$ $5.13589615141373 \quad \mathrm{c}$ $7.78733298568101 \quad \mathrm{c}$ $3.05883238711575 \quad \mathrm{c}$ $4.69084411121924 \quad c$ $5.08750099610365 \quad \mathrm{c}$ $7.78089944231988 \quad \mathrm{c}$ $3.07186361193826 \quad \mathrm{c}$ 4.65936148712729 c $5.03838084628587 \quad \mathrm{c}$ $7.70823089959480 \quad \mathrm{c}$ $2.98732578837446 \quad c$ $4.65989315961227 \quad \mathrm{c}$ $5.07218114060211 \quad \mathrm{c}$ $7.76091182864903 \quad \mathrm{c}$ $3.05923362610432 \quad c$ $4.66115224083753 \quad \mathrm{c}$ $5.02560730742336 \quad c$ $7.69491793556298 \quad \mathrm{c}$ $2.96904874745636 \quad \mathrm{c}$ $5.08790246133713 \mathrm{~h}$ $9.28466068844077 \mathrm{~h}$ $8.13381652394635 \mathrm{~h}$ $7.97380213711190 \mathrm{~h}$ $3.38423499889119 \mathrm{~h}$ 


\begin{tabular}{|c|c|}
\hline 1369761622593 & 6.32903723264536 \\
\hline 0.67598621186051 & 6.21208299400270 \\
\hline-6.09726286952097 & -0.08560418814127 \\
\hline-4.60533625311883 & 1.36955902418280 \\
\hline-7.38191214672593 & 2.95316089588334 \\
\hline-4.39526567240011 & 4.52916750202423 \\
\hline-7.97635259365836 & 3.79120586409698 \\
\hline-4.98396373482037 & 5.31865268332232 \\
\hline-5.74426836280193 & 2.67096214271863 \\
\hline-1.90406934211398 & -5.90308263280520 \\
\hline-2.91652521869397 & -3.99134718874225 \\
\hline-5.23619507895984 & -6.17293106747286 \\
\hline-5.84412049862816 & -2.85457830360433 \\
\hline-6.13288421318753 & -6.50270149623969 \\
\hline-6.66917555124427 & -3.18938317157034 \\
\hline-4.34292591488403 & -4.72578994117302 \\
\hline 4.91048384246817 & -3.70427708507197 \\
\hline 2.77893921245848 & -4.01623172232570 \\
\hline 4.13799863112785 & -6.91840356734012 \\
\hline 0.79422334325264 & -6.46760736397451 \\
\hline 4.20650298141701 & -7.95332380881111 \\
\hline 3.08326327370933 & -5.74571902392339 \\
\hline 0.89178849419408 & -7.45167240047465 \\
\hline 4.91859113993299 & 3.45768053382217 \\
\hline 7.78715417150743 & 1.73199623358459 \\
\hline 4.63294037292869 & 1.33539856581534 \\
\hline 6.32558910526921 & -1.31114428483636 \\
\hline 8.67854157549512 & 1.46519303456638 \\
\hline 6.17360927181637 & 1.03417880240530 \\
\hline 7.18242758050074 & -1.54544906969918 \\
\hline-0.02722674976402 & -0.00303169612724 \\
\hline-0.07190677708679 & -2.43691131810221 \\
\hline-0.11217641233927 & -5.30785453910482 \\
\hline-1.05946893702524 & -6.03937422804606 \\
\hline-1.48854146309969 & -6.87595092199085 \\
\hline 2.17616220414092 & -0.92792226926449 \\
\hline 4.90299842262728 & -1.82798969714141 \\
\hline 5.32805192033837 & -2.89886203669655 \\
\hline 5.94875165241035 & -3.66708941425530 \\
\hline 1.42689924272476 & 1.72826178148845 \\
\hline 3.15067380296720 & 4.02309651245410 \\
\hline 4.38818165312598 & 4.06088778016771 \\
\hline 5.15106978932849 & 4.47233518780433 \\
\hline-1.27502399555693 & 1.83953077973139 \\
\hline-2.94520965452140 & 4.17298989258436 \\
\hline-2.61584954742971 & 5.31182385656972 \\
\hline-2.75559107045439 & 6.25009784825868 \\
\hline-2.21493509588128 & -0.73240721489459 \\
\hline-4.95623755886190 & -1.59096287190941 \\
\hline-5.97151245867558 & -0.97804182468658 \\
\hline-6.83510799969295 & -0.69436118762774 \\
\hline
\end{tabular}

\begin{tabular}{|c|c|}
\hline & \\
\hline 321090 & \\
\hline 4953882933149 & \\
\hline 23685379529904 & \\
\hline 11084492586592 & \\
\hline .05091113126847 & \\
\hline 3. 38943824459400 & \\
\hline .11230077262030 & \\
\hline 1.14148158174128 & \\
\hline 4.93566259518508 & \\
\hline 9.19289579771851 & \\
\hline 8.03396744720106 & \\
\hline 7.93575189522030 & \\
\hline 3.29484983299154 & \\
\hline 3.00148236228813 & \\
\hline .07266692160888 & \\
\hline 794 & \\
\hline 3616 & \\
\hline 582 & \\
\hline 544 & \\
\hline 193 & \\
\hline 97 & \\
\hline 370 & \\
\hline 22 & \\
\hline 0 & \\
\hline 279 & \\
\hline 9632 & \\
\hline 39974 & \\
\hline 1.05 & \\
\hline 3.00 & \\
\hline-0.0 & \\
\hline-4.6 & \\
\hline-5.0 & \\
\hline-7.7 & \\
\hline-3.02 & \\
\hline-4.656 & \\
\hline-5.04 & \\
\hline-7.73 & \\
\hline-3.03 & \\
\hline-4.69 & \\
\hline-5.08 & \\
\hline-7.73 & \\
\hline-3.00809 & \\
\hline-4.72 & \\
\hline-5.14 & \\
\hline-7.8187342 & \\
\hline-3.10109981083867 & \\
\hline-4.66221762224364 & \\
\hline-5.02129409855102 & \\
\hline 050634 & \\
\hline & \\
\hline
\end{tabular}




$\begin{array}{rr}1.87824546196877 & -5.91393531262788 \\ -0.08546083018154 & -4.92840252634418 \\ -0.67867475899919 & -8.06837688630872 \\ -3.11656736916968 & -5.73893387848086 \\ -1.16996005515521 & -8.91737525054628 \\ -3.55558297034944 & -6.56360721942848 \\ -0.75979547512283 & -6.45008929936230 \\ 6.09281608642198 & -0.12369764440811 \\ 4.58363195526458 & -1.60033692778322 \\ 7.37835774607656 & -3.15570010665362 \\ 4.40515305666292 & -4.75610709221341 \\ 7.99299218377223 & -3.98016226363159 \\ 5.01534453116035 & -5.53603180548276 \\ 5.74814757058665 & -2.89071890149863 \\ 1.89746674893655 & 5.68495783598556 \\ 2.96959838319295 & 3.73222583788446 \\ 5.26980122986422 & 5.93052911621814 \\ 5.88374565260059 & 2.61468525261907 \\ 6.09652477194240 & 6.31407952402317 \\ 6.63900703579011 & 3.00480511775049 \\ 4.27984288506047 & 4.55236858727496 \\ -4.91010412577080 & 3.48956221168457 \\ -2.76884492155830 & 3.83444745405333 \\ -4.10922606795138 & 6.73761121949831 \\ -0.76876864555749 & 6.26237406688920 \\ -4.20180079016451 & 7.72429433380993 \\ -3.11724982371621 & 5.47947036990920 \\ -0.89240863301090 & 7.19740007987480 \\ -4.92714108592142 & -3.66855204716882 \\ -7.79104567647782 & -1.96910103941060 \\ -4.63400103741355 & -1.60625165643104 \\ -6.31249423785856 & 1.06654542769837 \\ -8.68488223226336 & -1.63532984830278 \\ -6.17802697197551 & -1.20060209821216 \\ -7.16908520945940 & 1.36784377837036\end{array}$
\$end

\section{$5.5 \mathrm{Bk}$}

\$coord

$$
\begin{array}{r}
-0.24975809805562 \\
-0.65508347371328 \\
0.13639359490708 \\
0.49820105319723 \\
-2.26771980785265 \\
-5.09541249914367 \\
-5.66641847391153 \\
-6.41551647534408 \\
-1.14159259496864 \\
-2.48619350484097
\end{array}
$$

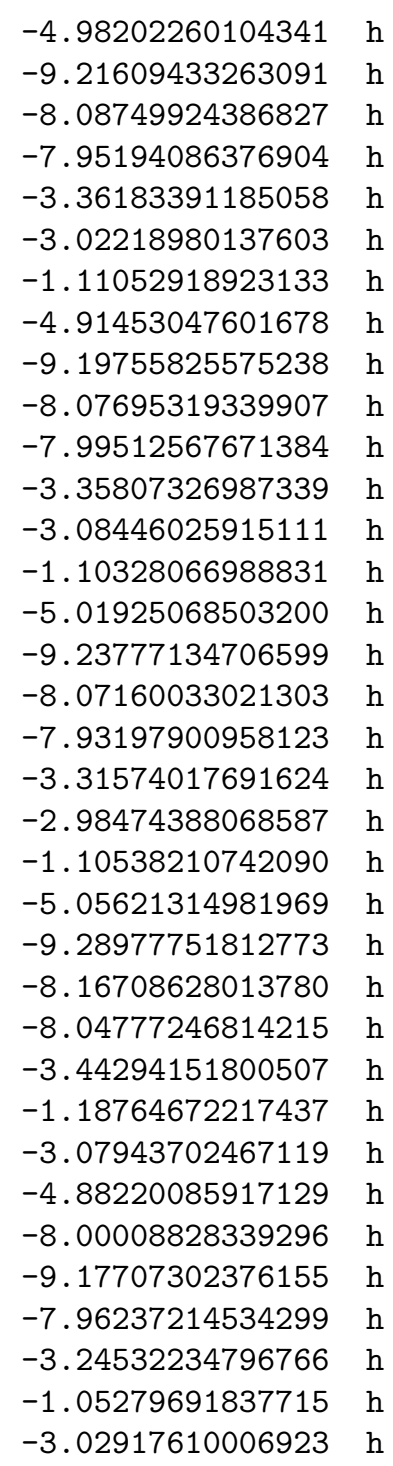

.

.

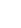

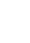

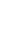

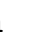

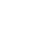

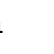

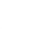

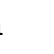

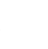

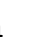




\begin{tabular}{|c|c|}
\hline-3.63589877430146 & -4.77775511317151 \\
\hline-4.45083885083101 & -5.29752877965002 \\
\hline 1.57206868075741 & -1.71326379316214 \\
\hline 3.56812805885584 & -3.77567120662265 \\
\hline 3.42706864836106 & -4.93100684958477 \\
\hline 3.67170134959999 & -5.88289904054727 \\
\hline 2.12309345812212 & 0.96146035322506 \\
\hline 4.70048927644385 & 2.22356539915830 \\
\hline 5.75178860422469 & 1.73041806813685 \\
\hline 6.74024632613620 & 1.67463215442728 \\
\hline-2.71306894151965 & 5.45143440976748 \\
\hline-0.66875738959213 & 4.74011232736788 \\
\hline-0.56047645 & 7.94246383183787 \\
\hline 2.21262417947798 & 6.02139095463371 \\
\hline-0.12609466918271 & 8.89187748498054 \\
\hline 2.58799638317299 & 6.92182287755200 \\
\hline-0.13552827522984 & 6.41541808456697 \\
\hline-6.01481421129677 & -0.89971456816517 \\
\hline-4.72181509773258 & 0.83811623182356 \\
\hline-7.72997022879933 & 1.92679974692029 \\
\hline-5.04634579801321 & 3.97068614860871 \\
\hline-8.48223153223791 & 2.60986314094395 \\
\hline-5.77382607962083 & 4.59065331631091 \\
\hline-6.11118505481507 & 1.83897242550493 \\
\hline-0.99754966166320 & -6.00349038219307 \\
\hline-2.24739597896291 & -4.24052913923903 \\
\hline-4.21990692478539 & -6.75822125950089 \\
\hline-5.32513775562046 & -3.57041142326013 \\
\hline-5.09373848523599 & -7.26286030223756 \\
\hline-6.14620172098687 & -4.07537180141422 \\
\hline-3.63235106874171 & -5.23448020001221 \\
\hline 5.40926297371394 & -2.80911449427819 \\
\hline 3.34240409140396 & -3.44074557193827 \\
\hline 5.13037018417928 & -6.09649658271789 \\
\hline 1.75741002664515 & -6.16537393801171 \\
\hline 5.34015963160447 & -7.10207856475982 \\
\hline 3.86912767933082 & -5.09378481181056 \\
\hline 1.98459701851383 & -7.11608312184725 \\
\hline 4.34912791990900 & 4.27338098334037 \\
\hline 7.38287590767919 & 2.99365866922734 \\
\hline 4.30470040351776 & 2.10489124112011 \\
\hline 6.41432123287381 & -0.23763908033624 \\
\hline 8.41653295882287 & 2.88029614707384 \\
\hline 6.05632769728606 & 2.11506960721004 \\
\hline 7.38695000352566 & -0.31254661454901 \\
\hline-0.00001514014672 & 0.00002123094405 \\
\hline 0.25006567231995 & -2.31166932598134 \\
\hline 0.65576891221954 & -5.15319681928782 \\
\hline-0.13518570438280 & -6.00312864772652 \\
\hline-0.49693235544552 & -6.92282984653511 \\
\hline 2.26776280852834 & -0.47107211427431 \\
\hline
\end{tabular}

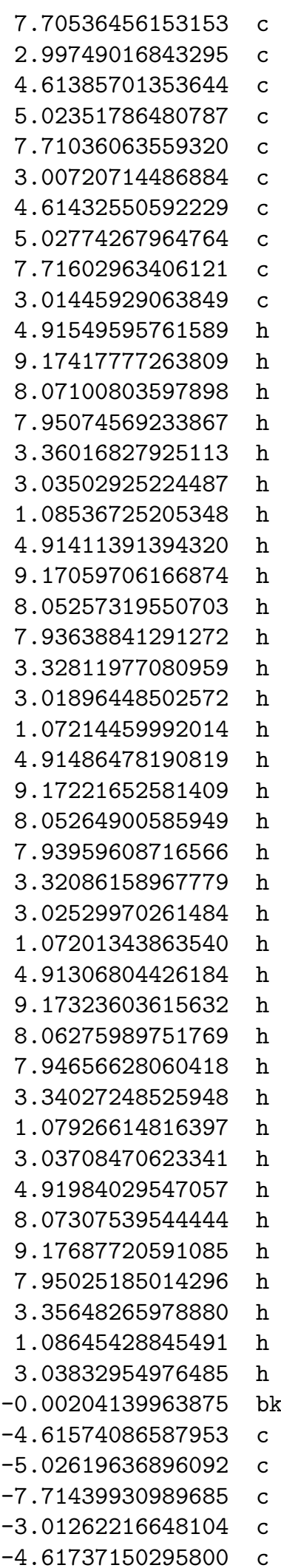




\begin{tabular}{|c|c|c|}
\hline 5.09570773694000 & -0.96493078320455 & -5.02134200083617 \\
\hline 5.66832849983826 & -1.98861762507637 & -7.70401412543391 \\
\hline 6.41407838750149 & -2.60121122208997 & -2.99668139995227 \\
\hline 1.14130801848697 & 2.01537513679449 & -4.61978237228610 \\
\hline 2.48561781818109 & 4.55185563165177 & -5.02272979620699 \\
\hline 3.63640045592734 & 4.78052194890377 & -7.70531732386701 \\
\hline 4.44891649043641 & 5.29695111120308 & -2.99644035151602 \\
\hline-1.57226123888994 & 1.71302173320704 & -4.61653607987433 \\
\hline-3.56869987530535 & 3.77537189135730 & -5.02433196169373 \\
\hline-3.42896816695203 & 4.93232071540619 & -7.71049869639199 \\
\hline-3.67136371429078 & 5.88117503035819 & -3.00647507643659 \\
\hline-2.12292735750512 & -0.96173452922736 & -4.61700975767851 \\
\hline-4.70036806819484 & -2.22423153643222 & -5.02867699817383 \\
\hline-5.75341841410743 & -1.73180159232954 & -7.71635926226433 \\
\hline-6.73858346869191 & -1.67475723059796 & -3.01398373316066 \\
\hline 2.71381069071258 & -5.45100925712788 & -4.91596089109590 \\
\hline 0.67177210893882 & -4.74500953296499 & -9.17526633749124 \\
\hline 0.56029394534697 & -7.94600831217708 & -8.06841654071715 \\
\hline-2.21131781068640 & -6.02249154795286 & -7.95187533406873 \\
\hline 0.12918129285736 & -8.89028771447324 & -3.35611962246603 \\
\hline-2.58671770396918 & -6.92205935160198 & -3.03477028197193 \\
\hline 0.13563381695992 & -6.41076845627285 & -1.08474306367585 \\
\hline 6.01510953099569 & 0.90023980264248 & -4.91463551698314 \\
\hline 4.72637530034712 & -0.83681017511034 & -9.17173891446201 \\
\hline 7.73228646357891 & -1.92887759176595 & -8.05088079054180 \\
\hline 5.04626859914004 & -3.96985634322320 & -7.93755311049971 \\
\hline 8.48124525761499 & -2.60806209546855 & -3.32493924750194 \\
\hline 5.77340773306465 & -4.59027893980158 & -3.01993485255611 \\
\hline 6.10679973252796 & -1.83892892230603 & -1.07200261905870 \\
\hline 0.99662050422619 & 6.00364017965083 & -4.91519896596344 \\
\hline 2.24792595423462 & 4.24596430174229 & -9.17317411081215 \\
\hline 4.22190559857846 & 6.76097679792876 & -8.05009014967737 \\
\hline 5.32483277236672 & 3.57220905754358 & -7.94043266631111 \\
\hline 5.09023186463275 & 7.26330718616862 & -3.31665961706533 \\
\hline 6.14533579093376 & 4.07628362074830 & -3.02520496493100 \\
\hline 3.62967863570837 & 5.23052642236407 & -1.07134865081754 \\
\hline-5.40970558839065 & 2.80859519455332 & -4.91362460801705 \\
\hline-3.34678919897756 & 3.44282010964142 & -9.17429141673662 \\
\hline-5.13161111390905 & 6.09942509880061 & -8.06069040045756 \\
\hline-1.75844252248991 & 6.16541132266020 & -7.94736669798470 \\
\hline-5.34140184523441 & 7.09908747475424 & -3.33621605479292 \\
\hline-3.86539162211831 & 5.09049458572662 & -1.07879164504759 \\
\hline-1.98539965857925 & 7.11588308788966 & -3.03777222434339 \\
\hline-4.34881827104396 & -4.27399914763103 & -4.92046267936903 \\
\hline-7.38591526432989 & -2.99381319194932 & -8.07127011623779 \\
\hline-4.30777734835798 & -2.10852375678156 & -9.17806121328237 \\
\hline-6.41432875254242 & 0.23671658508136 & -7.95134778941819 \\
\hline-8.41446645719570 & -2.88178295790998 & -3.35315968755278 \\
\hline-6.05247550359633 & -2.11306316312477 & -1.08622943823107 \\
\hline-7.38652768715970 & 0.31200162520258 & -3.03893847271793 \\
\hline
\end{tabular}




\subsection{No}

\$coord

$-0.27826920780649$

$-0.67416974187278$

0.13222246740360

0.48634469710581

$-2.26277005813402$

$-5.08084181722281$

$-5.63886544707889$

$-6.37687500904413$

$-1.10657779503526$

$-2.46625077596117$

$-3.63012713535026$

$-4.42076403215331$

1.56292886030175

3. 57099116400090

3.42758674191564

3. 65670390818909

2. 09451507283351

4.65979515751871

5.71196964041841

6.66785046921710

$-2.72992318826884$

$-0.66294173032180$

$-0.55821315599064$

2.21030187335636

$-0.14027524636821$

2.57599550205884

$-0.12936263487068$

$-6.00290408359786$

$-4.66246765246307$

$-7.69752351429647$

$-5.02998275056872$

$-8.44857672406168$

$-5.74194724621644$

$-6.02165509480493$

$-0.99231188440952$

$-2.24322268463347$

$-4.21831601710620$

$-5.31723588111110$

$-5.06728949203093$

$-6.11287056175336$

$-3.58657432149677$

5.41061930979224

3.31698406029488

5.13479413633119

1. 76112603366274

5.33483399390263

3. 80681931030171

1. 97170247498000
2.32720769975513

5. 16399205233908

6.00264983754329

6.90753741573134

0.48449265020989

0.95168608948398

1. 96478503248503

2. 60484422607832

$-2.03987683227061$

$-4.55478562130416$

$-4.76082967952443$

$-5.26147128656651$

$-1.73120114319117$

$-3.77238817063166$

$-4.91985761836151$

$-5.86982430298431$

0.98056703529954

2. 23127670898998

1. 72361734058638

1. 64511381921471

5.47389480030512

4.72872477627999

7.94296252076526

6.01726232229976

8.88074342654326

6.90338925819345

6.35190210111909

$-0.90992671890907$

0.82096059364627

1. 88127917631667

3. 95070076825035

2.59402509134224

4.59399032439660

1.86821737309306

$-6.02122096383197$

$-4.20948382052215$

$-6.73713266527783$

$-3.54779771643420$

$-7.23192217210877$

$-4.03586356199817$

$-5.15157108542052$

$-2.80613716264505$

$-3.42037986923668$

$-6.07270774382593$

$-6.16048144839076$

$-7.08442147941283$

$-5.06242641681199$

$-7.10456124026764$
$4.56511704942405 \quad \mathrm{C}$

$4.97642482475319 \quad \mathrm{c}$

$7.66106776232896 \quad \mathrm{c}$

$2.94765176262995 \quad \mathrm{c}$

$4.56653617929044 \quad \mathrm{C}$

$4.96775504073510 \quad \mathrm{c}$

$7.65862703937690 \quad \mathrm{C}$

$2.94252458906145 \quad \mathrm{c}$

$4.56853996788375 \quad \mathrm{c}$

$4.96603527255382 \quad \mathrm{c}$

$7.64447234129536 \quad \mathrm{c}$

$2.91907098504092 \quad \mathrm{c}$

$4.56692916429541 \quad \mathrm{C}$

$4.97382160042671 \quad \mathrm{c}$

$7.66203587406832 \quad \mathrm{c}$

$2.94886904236888 \quad \mathrm{c}$

$4.56700296812170 \quad \mathrm{c}$

$4.96464940302030 \quad \mathrm{c}$

$7.65163402694190 \quad \mathrm{c}$

$2.92969031849261 \quad \mathrm{c}$

$4.86926905232900 \mathrm{~h}$

$9.11503850499573 \mathrm{~h}$

$8.03650349314630 \mathrm{~h}$

$7.88321323626177 \mathrm{~h}$

$3.25365609582432 \mathrm{~h}$

$2.98552791590085 \mathrm{~h}$

$1.02844514521864 \mathrm{~h}$

$4.84792266162982 \mathrm{~h}$

$9.10973473462441 \mathrm{~h}$

$8.02730864527027 \mathrm{~h}$

$7.88155116108215 \mathrm{~h}$

$3.23591440986036 \mathrm{~h}$

$3.00383172951339 \mathrm{~h}$

$1.01858365440199 \mathrm{~h}$

$4.86438145621182 \mathrm{~h}$

$9.10757873915511 \mathrm{~h}$

$8.00501199642827 \mathrm{~h}$

$7.86092872942092 \mathrm{~h}$

$3.19771759463175 \mathrm{~h}$

$2.96403988785902 \mathrm{~h}$

$1.00438880064191 \mathrm{~h}$

$4.85523085371433 \mathrm{~h}$

$9.11399619382389 \mathrm{~h}$

$8.03411026535891 \mathrm{~h}$

$7.88842350220078 \mathrm{~h}$

$3.24573268900122 \mathrm{~h}$

$1.02633527594457 \mathrm{~h}$

$3.00278095473670 \mathrm{~h}$ 


\begin{tabular}{|c|c|c|}
\hline 4.32589298357630 & 4.28260937509922 & 4.85297019369632 \\
\hline 7.33689674578461 & 2.99155889029821 & 8.01554049282225 \\
\hline 4.25660906276842 & 2.08294348303447 & 9.10767005169422 \\
\hline 6.37711394280614 & -0.24446192402499 & 7.86975659093486 \\
\hline 8.34161401620996 & 2.86816019152914 & 3.21265022812750 \\
\hline 5.93919816075492 & 2.03364736860582 & 1.00892698766028 \\
\hline 7.32012772559093 & -0.33842461035207 & 2.99025923151825 \\
\hline-0.00006379994914 & -0.00004684255260 & -0.00015204254881 \\
\hline 0.27827600192187 & -2.32736014394769 & -4.56536240140284 \\
\hline 0.67425639049044 & -5.16417649404522 & -4.97641465780329 \\
\hline-0.13223204597596 & -6.00309025814598 & -7.66094775610226 \\
\hline-0.48638931819049 & -6.90751181478377 & -2.94751006932772 \\
\hline 2.26271354248906 & -0.48457166824454 & -4.56678836051850 \\
\hline 5.08081604715327 & -0.95158517552000 & -4.96774537737454 \\
\hline 5.63945187986140 & -1.96412534905058 & -7.65867657883814 \\
\hline 6.37677703027172 & -2.60459672886124 & -2.94235866420143 \\
\hline 1.10647284877902 & 2.03975134396448 & -4.56879868288016 \\
\hline 2.46610522058785 & 4.55476494739138 & -4.96573668105534 \\
\hline 3.62962002355841 & 4.76183067014577 & -7.64423486693460 \\
\hline 4.42066561778797 & 5.26105145041136 & -2.91869481180542 \\
\hline-1.56302160550315 & 1.73100082337526 & -4.56739867315651 \\
\hline-3.57107442402679 & 3.77215542472653 & -4.97453521980989 \\
\hline-3.42663410757425 & 4.92030713122778 & -7.66239680269625 \\
\hline-3.65730178534421 & 5.86915268695109 & -2.94913975501736 \\
\hline-2.09452971957111 & -0.98077357915474 & -4.56736464996716 \\
\hline-4.65980915102460 & -2.23152232030920 & -4.96475240720059 \\
\hline-5.71213705901897 & -1.72423616817576 & -7.65174730141752 \\
\hline-6.66768707576173 & -1.64515083210496 & -2.92967511331593 \\
\hline 2.73001323980436 & -5.47408447873978 & -4.86924430731172 \\
\hline 0.66300362533182 & -4.72944338813146 & -9.11512112057325 \\
\hline 0.55798977785077 & -7.94352070211514 & -8.03614777626598 \\
\hline-2.21032278074636 & -6.01752642845885 & -7.88298846094686 \\
\hline 0.14050619246761 & -8.88070568911267 & -3.25301779794696 \\
\hline-2.57603865538432 & -6.90361473624605 & -2.98582039730678 \\
\hline 0.12880284680561 & -6.35148013720450 & -1.02824944128968 \\
\hline 6.00261735852922 & 0.91014163788302 & -4.84756114776799 \\
\hline 4.66328568510385 & -0.82003183404259 & -9.10972178528960 \\
\hline 7.69818026445516 & -1.88030408826722 & -8.02685271451744 \\
\hline 5.03081485006606 & -3.95006511003047 & -7.88220360628592 \\
\hline 8.44853210526746 & -2.59338683500245 & -3.23533417120596 \\
\hline 5.74223884453698 & -4.59386842529164 & -3.00380227690385 \\
\hline 6.02102131369703 & -1.86802598845886 & -1.01849507405917 \\
\hline 0.99208659544335 & 6.02108662057565 & -4.86343019927279 \\
\hline 2.24244340391588 & 4.21124465223104 & -9.10735609412642 \\
\hline 4.21782039107746 & 6.73827600411133 & -8.00395281480225 \\
\hline 5.31662676394008 & 3.54879073127658 & -7.86154292083202 \\
\hline 5.06707361016232 & 7.23160473780047 & -3.19685767170330 \\
\hline 6.11288948071021 & 4.03562035714724 & -2.96395778279974 \\
\hline 3.58649324983207 & 5.15064048677216 & -1.00403019381668 \\
\hline-5.41073885040892 & 2.80587547959113 & -4.85683690873325 \\
\hline-3.31601247326506 & 3.42120960137451 & -9.11475165781056 \\
\hline
\end{tabular}




$$
\begin{aligned}
& -5.13341246021512 \\
& -1.75978454383582 \\
& -5.33569593554576 \\
& -3.80721678034022 \\
& -1.97252587431907 \\
& -4.32589298826891 \\
& -7.33707315478177 \\
& -4.25685945243874 \\
& -6.37729510973891 \\
& -8.34138020383065 \\
& -5.93876831474768 \\
& -7.32012915913731
\end{aligned}
$$
\$end

\section{$5.7 \quad \mathrm{Lr}$}

$\$$ coord

$$
\begin{array}{rr}
-0.25800656556899 & 2.31667690503902 \\
-0.66330362980540 & 5.14891788321622 \\
0.09311458280883 & 5.92502133093218 \\
0.53894436885512 & 6.94938234195469 \\
-2.27835846312030 & 0.46987123853550 \\
-5.09734257707061 & 0.95967998048710 \\
-5.60546748372483 & 1.92008186354695 \\
-6.43652521656588 & 2.65709529104045 \\
-1.14618026782282 & -2.02194011675716 \\
-2.48412080533181 & -4.55152827001557 \\
-3.55745505532220 & -4.73977856218346 \\
-4.50996391552525 & -5.29653947905565 \\
1.57362275537520 & -1.71578731437335 \\
3.56538451402942 & -3.76994793352333 \\
3.41061925570837 & -4.84475076258108 \\
3.64911132863367 & -5.93121939787642 \\
2.12270805518628 & 0.96582619257426 \\
4.69152858037637 & 2.22572828866296 \\
5.66295820056901 & 1.74733008489807 \\
6.77512269324745 & 1.63744102706537 \\
-2.71733467307625 & 5.45383815283104 \\
-0.68956788771993 & 4.59720611704210 \\
-0.64111581401086 & 7.83999676632925 \\
2.16817957313686 & 5.97539142671757 \\
-0.15025769605222 & 8.90053226551890 \\
2.62394147608404 & 6.99565998107561 \\
0.02309084334537 & 6.41465565231205 \\
-6.02159770077307 & -0.89976846381995 \\
-4.58618092075630 & 0.76623209019514 \\
-7.65423188303248 & 1.81307180965001 \\
-5.01353120199817 & 3.90952101906972 \\
-8.50579904390686 & 2.60146566585478 \\
-5.83972894821929 & 4.65534849719630
\end{array}
$$

$$
\begin{array}{r}
6.07372601798441 \\
6.16055888939501 \\
7.08345852928199 \\
5.06130093506576 \\
7.10423265637150 \\
-4.28283768557913 \\
-2.99225878760192 \\
-2.08385768718337 \\
0.24380800651957 \\
-2.86839141752399 \\
-2.03335389965988 \\
0.33832837744171
\end{array}
$$

$$
\begin{array}{ll}
-8.03465405242809 & \mathrm{~h} \\
-7.88789816638125 & \mathrm{~h} \\
-3.24572886795673 & \mathrm{~h} \\
-1.02678940588495 & \mathrm{~h} \\
-3.00282091442015 & \mathrm{~h} \\
-4.85283693741130 & \mathrm{~h} \\
-8.01530969475030 & \mathrm{~h} \\
-9.10778553384810 & \mathrm{~h} \\
-7.87024567423724 & \mathrm{~h} \\
-3.21224705364916 & \mathrm{~h} \\
-1.00893495649284 & \mathrm{~h} \\
-2.99042791534889 & \mathrm{~h}
\end{array}
$$

$\begin{array}{ll}4.35419602274566 & \mathrm{c} \\ 4.80513065422041 & \mathrm{c} \\ 7.52343318495686 & \mathrm{c} \\ 2.85260178794597 & \mathrm{c} \\ 4.35520404252219 & \mathrm{c} \\ 4.80388658970980 & \mathrm{c} \\ 7.52082534892056 & \mathrm{c} \\ 2.84813980857444 & \mathrm{c} \\ 4.35593802725422 & \mathrm{c} \\ 4.80205590518167 & \mathrm{c} \\ 7.51773061245348 & \mathrm{c} \\ 2.84232268655511 & \mathrm{c} \\ 4.35454689513892 & \mathrm{c} \\ 4.80331201540895 & \mathrm{c} \\ 7.52055728745559 & \mathrm{c} \\ 2.84796775240612 & \mathrm{c} \\ 4.35403466537733 & \mathrm{c} \\ 4.80492553351920 & \mathrm{c} \\ 7.52372134600404 & \mathrm{c} \\ 2.85250174947667 & \mathrm{c} \\ 4.66100283782660 & \mathrm{~h} \\ 8.93427486302920 & \mathrm{~h} \\ 7.94172674381638 & \mathrm{~h} \\ 7.76433502303360 & \mathrm{~h} \\ 3.16235476048388 & \mathrm{~h} \\ 2.98244370951835 & \mathrm{~h} \\ 0.89830940022187 & \mathrm{~h} \\ 4.66032982878449 & \mathrm{~h} \\ 8.93357656186588 & \mathrm{~h} \\ 7.93598342314022 & \mathrm{~h} \\ 7.76187511179194 & \mathrm{~h} \\ 3.15304970820621 & \mathrm{~h} \\ 2.97830447495475 & \mathrm{~h}\end{array}$ 


\begin{tabular}{|c|c|c|}
\hline-6.08210135247668 & 2.00082827536588 & 0.89472064105930 \\
\hline-1.00187865731887 & -6.00601164901895 & 4.65859525369862 \\
\hline-2.14544887331679 & -4.13030745064598 & 8.93230946817790 \\
\hline-4.09111468014829 & -6.72124316195949 & 7.93045274232534 \\
\hline-5.26485201374951 & -3.55986510549885 & 7.75819672707190 \\
\hline-5.09551557704590 & -7.28309843091072 & 3.13939377800583 \\
\hline-6.22705563609376 & -4.11288757834852 & 2.97523015701629 \\
\hline-3.77439846349707 & -5.15487571397150 & 0.88982861829443 \\
\hline 5.40667559614319 & -2.81012401782292 & 4.65887026764193 \\
\hline 3.26663796700850 & -3.31167616910669 & 8.93318159458278 \\
\hline 5.12912657831153 & -5.96497261435972 & 7.93643439388489 \\
\hline 1.76004013456114 & -6.10327950801921 & 7.76165596923504 \\
\hline 5.35773135748314 & -7.10059417110900 & 3.14995890706375 \\
\hline 3.74259746330026 & -5.19288316461761 & 0.89415799164439 \\
\hline 1.99356273582542 & -7.19912397688808 & 2.98261536652450 \\
\hline 4.34791524570494 & 4.27381428345359 & 4.66040869081550 \\
\hline 7.25673003576157 & 3.03822240183636 & 7.94169199619749 \\
\hline 4.15802177880055 & 2.08140399484709 & 8.93436313853215 \\
\hline 6.35303171117406 & -0.21017614811780 & 7.76535961763117 \\
\hline 8.41651723109712 & 2.89825579216663 & 3.158827 \\
\hline 6.10503146554379 & 1.95812319236060 & 0.8981907 \\
\hline 7.46611297128455 & -0.33020561803655 & 2.98559665937735 \\
\hline-0.00028797169094 & 0.00014040395960 & -0.00023013590757 \\
\hline 0.25807862197157 & -2.31668416316222 & -4.35446892076259 \\
\hline 0.66357422135944 & -5.14895040656072 & -4.804920 \\
\hline-0.09186533952424 & -5.92562497831230 & -7.52332268981479 \\
\hline-0.53891186502096 & -6.94917245550785 & -2.85231554287038 \\
\hline 2.27835167673107 & -0.46980258911369 & -4.35537547719172 \\
\hline 5.09739750943943 & -0.95949597991355 & -4.80376717681247 \\
\hline 5.60570895399604 & -1.92010776392891 & -7.52058964542671 \\
\hline 6.43639410859543 & -2.65689219863252 & -2.84787183327438 \\
\hline 1.14605022753200 & 2.02195573826293 & -4.35636415380728 \\
\hline 2.48388248885947 & 4.55165327675406 & -4.80210360364100 \\
\hline 3.55719974844966 & 4.74055499258343 & -7.51773148516494 \\
\hline 4.50953313528601 & 5.29651816671534 & -2.84211333347912 \\
\hline-1.57372453141307 & 1.71568187747211 & -4.35523639476806 \\
\hline-3.56558914336173 & 3.76977298346874 & -4.80376055178943 \\
\hline-3.41104689830489 & 4.84496258313194 & -7.52084811691981 \\
\hline-3.64936507196630 & 5.93069283923317 & -2.84802164654384 \\
\hline-2.12268416641422 & -0.96593502731516 & -4.35464476347896 \\
\hline-4.69146544050901 & -2.22595627288967 & -4.80536086940747 \\
\hline-5.66319267925832 & -1.74735203067107 & -7.52400265212219 \\
\hline-6.77480010834275 & -1.63760037989986 & -2.85266015042883 \\
\hline 2.71759244087422 & -5.45366277889478 & -4.66016339252000 \\
\hline 0.69137195258472 & -4.59814378461172 & -8.93416538446342 \\
\hline 0.64255421729742 & -7.84068355796339 & -7.94088174763162 \\
\hline-2.16684235611614 & -5.97606203638600 & -7.76501567838965 \\
\hline 0.15064356112715 & -8.90029861493432 & -3.16143238264830 \\
\hline-2.62387844536106 & -6.99574490772735 & -2.98261267835119 \\
\hline-0.02355899836074 & -6.41387293623495 & -0.89804391852188 \\
\hline 6.02160213538895 & 0.89997903404536 & -4.66023200901013 \\
\hline
\end{tabular}




$\begin{array}{rrrr}4.58680185479119 & -0.76616491480715 & -8.93353166469404 & \mathrm{~h} \\ 7.65454088428082 & -1.81341408425713 & -7.93547026807723 & \mathrm{~h} \\ 5.01351051798709 & -3.90947903376827 & -7.76160833342616 & \mathrm{~h} \\ 8.50573742372394 & -2.60082354482498 & -3.15222069886949 & \mathrm{~h} \\ 5.84002543692754 & -4.65525407855875 & -2.97842731218115 & \mathrm{~h} \\ 6.08130751421457 & -2.00092965624035 & -0.89447904867543 & \mathrm{~h} \\ 1.00152856854159 & 6.00600157924276 & -4.65842603494103 & \mathrm{~h} \\ 2.14513548293529 & 4.13158646035831 & -8.93246585651092 & \mathrm{~h} \\ 4.09095934929296 & 6.72210475863799 & -7.92989549807063 & \mathrm{~h} \\ 5.26453116646634 & 3.56061133509668 & -7.75853108947918 & \mathrm{~h} \\ 5.09473851300241 & 7.28326698559435 & -3.13858482919050 & \mathrm{~h} \\ 6.22683212802953 & 4.11318252635354 & -2.97526071702015 & \mathrm{~h} \\ 3.77388176699625 & 5.15416807974298 & -0.88969266369271 & \mathrm{~h} \\ -5.40681011403054 & 2.80980254517257 & -4.65939231153953 & \mathrm{~h} \\ -3.26741297206482 & 3.31206857621826 & -8.93369842930279 & \mathrm{~h} \\ -5.12953362707048 & 5.96536233540587 & -7.93630761496685 & \mathrm{~h} \\ -1.76040454781741 & 6.10340648664897 & -7.76199884220238 & \mathrm{~h} \\ -5.35826585478954 & 7.09980657047385 & -3.14944994118802 & \mathrm{~h} \\ -3.74239130969081 & 5.19193197021092 & -0.89433867862802 & \mathrm{~h} \\ -1.99405183309949 & 7.19891306036403 & -2.98268004193232 & \mathrm{~h} \\ -4.34783045108428 & -4.27404532899692 & -4.66097733723016 & \mathrm{~h} \\ -7.25714506757376 & -3.03807096740823 & -7.94180053299625 & \mathrm{~h} \\ -4.15847487928011 & -2.08157263801385 & -8.93484136111487 & \mathrm{~h} \\ -6.35309475484382 & 0.21023431658424 & -7.76551727809692 & \mathrm{~h} \\ -8.41609165390189 & -2.89869356268932 & -3.15841389441882 & \mathrm{~h} \\ -6.10430763912133 & -1.95784989294366 & -0.89840286127850 & \mathrm{~h} \\ -7.46606138297502 & 0.32994605027879 & -2.98596824478822 & \mathrm{~h} \\ \mathrm{n} & & & \end{array}$

\section{Orbital occupations for solvated $\left[\operatorname{An}\left(\mathrm{Cp}^{\mathrm{iPr} 5}\right)_{2}\right]^{+}$cations}

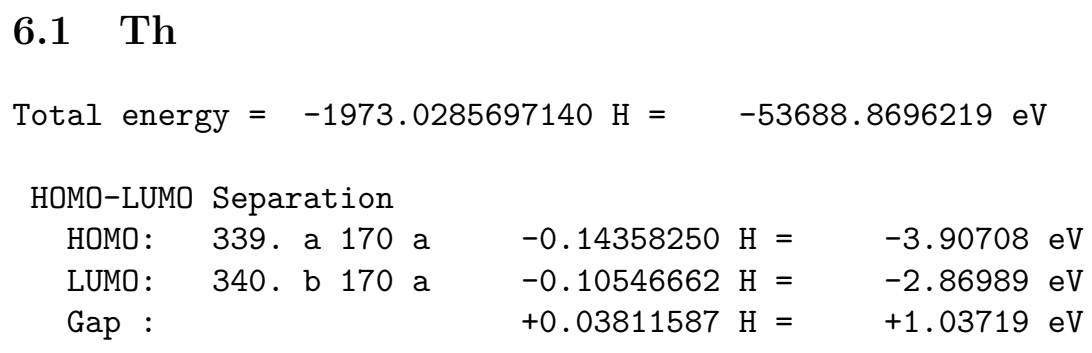

Number of MOs $=1598$, Electrons $=339.00$, Symmetry: $c 1$

Nr. Orbital Occupation Energy

356. a 179 a $-0.066284 \mathrm{H}=\quad-1.804 \mathrm{eV}$

355. a 178 a $-0.067374 \mathrm{H}=\quad-1.833 \mathrm{eV}$

354. b 177 a $\quad-0.079878 \mathrm{H}=\quad-2.174 \mathrm{eV}$

353. $\mathrm{b} 176 \mathrm{a} \quad-0.081589 \mathrm{H}=\quad-2.220 \mathrm{eV}$

352. $\mathrm{b} 175 \mathrm{a} \quad-0.082074 \mathrm{H}=\quad-2.233 \mathrm{eV}$

351. b 174 a $-0.083773 \mathrm{H}=\quad-2.280 \mathrm{eV}$ 


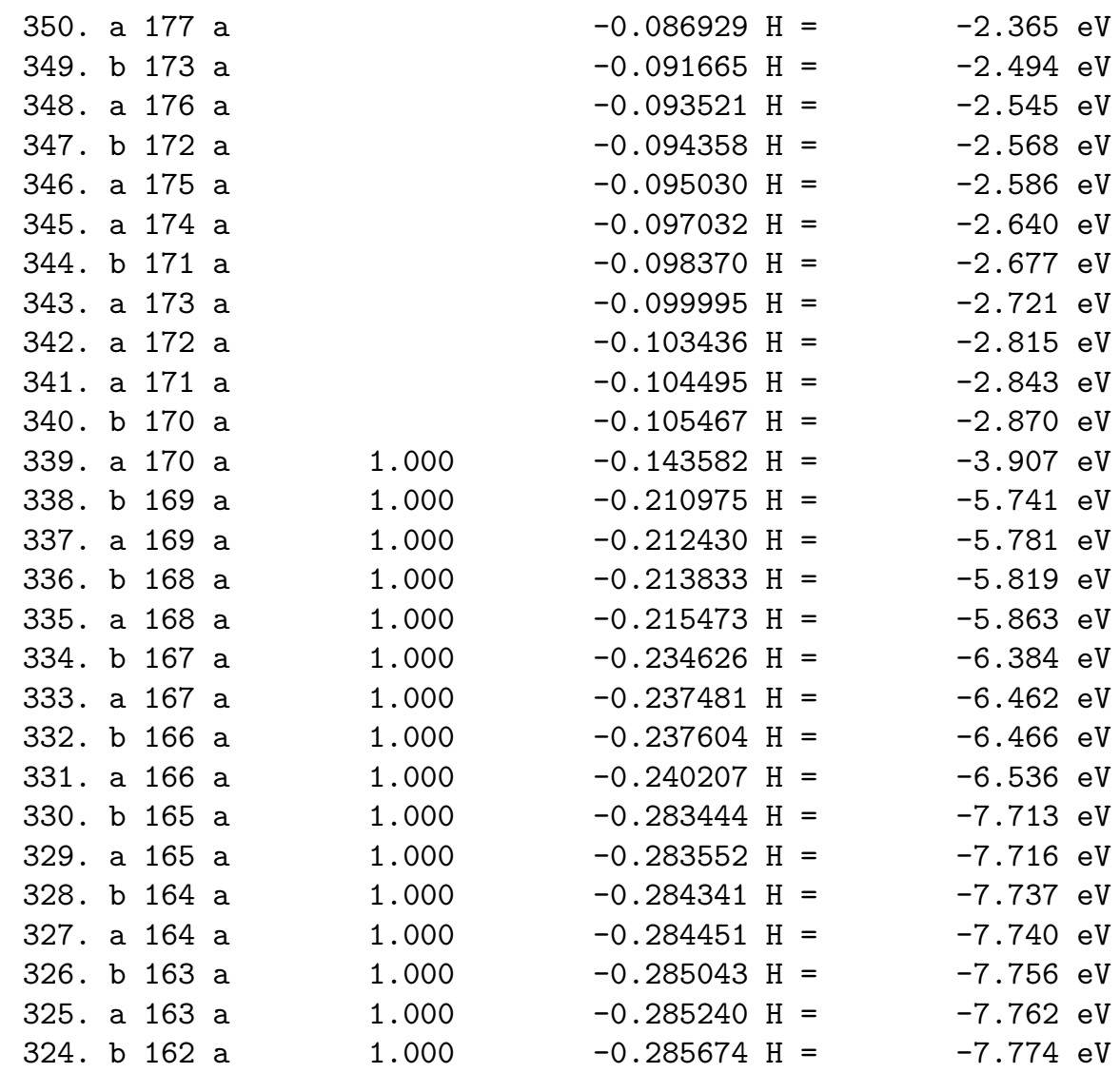

\section{$6.2 \mathrm{U}$}

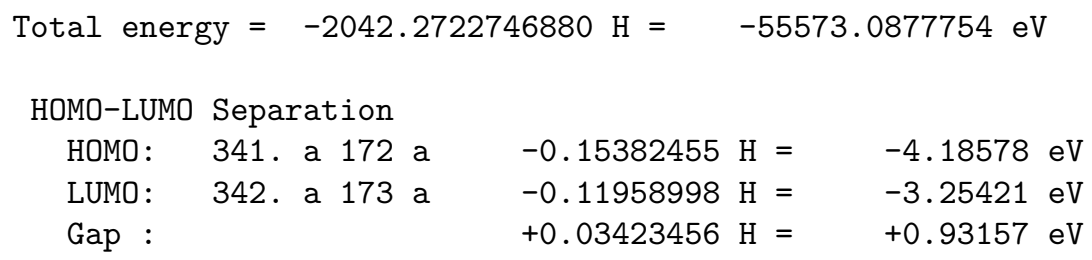

Number of MOs $=1598$, Electrons $=341.00$, Symmetry: c1

$\begin{array}{clll}\text { Nr. } & \text { Orbital } & \text { 0ccupation } & \text { Energy } \\ \text { 358. b } 179 \text { a } & -0.055220 \mathrm{H}= & -1.503 \mathrm{eV} \\ \text { 357. b } 178 \mathrm{a} & -0.062575 \mathrm{H}= & -1.703 \mathrm{eV} \\ \text { 356. b } 177 \mathrm{a} & -0.062651 \mathrm{H}= & -1.705 \mathrm{eV} \\ \text { 355. b } 176 \mathrm{a} & -0.076903 \mathrm{H}= & -2.093 \mathrm{eV} \\ \text { 354. b } 175 \mathrm{a} & -0.077013 \mathrm{H}= & -2.096 \mathrm{eV} \\ \text { 353. b } 174 \mathrm{a} & -0.088511 \mathrm{H}= & -2.408 \mathrm{eV} \\ \text { 352. b } 173 \mathrm{a} & -0.088607 \mathrm{H}= & -2.411 \mathrm{eV} \\ \text { 351. a } 179 \mathrm{a} & -0.091163 \mathrm{H}= & -2.481 \mathrm{eV} \\ \text { 350. a } 178 \mathrm{a} & -0.091198 \mathrm{H}= & -2.482 \mathrm{eV}\end{array}$




$\begin{array}{llll}\text { 349. b } 172 \mathrm{a} & -0.100239 \mathrm{H}= & -2.728 \mathrm{eV} \\ \text { 348. b } 171 \mathrm{a} & -0.100548 \mathrm{H}= & -2.736 \mathrm{eV} \\ \text { 347. b } 170 \mathrm{a} & -0.101048 \mathrm{H}= & -2.750 \mathrm{eV} \\ \text { 346. a } 177 \mathrm{a} & -0.115206 \mathrm{H}= & -3.135 \mathrm{eV} \\ \text { 345. a } 176 \mathrm{a} & -0.115361 \mathrm{H}= & -3.139 \mathrm{eV} \\ \text { 344. a } 175 \mathrm{a} & -0.119096 \mathrm{H}= & -3.241 \mathrm{eV} \\ \text { 343. a } 174 \mathrm{a} & & -0.119406 \mathrm{H}= & -3.249 \mathrm{eV} \\ \text { 342. a } 173 \mathrm{a} & & -0.119590 \mathrm{H}= & -3.254 \mathrm{eV} \\ \text { 341. a } 172 \mathrm{a} & 1.000 & -0.153825 \mathrm{H}= & -4.186 \mathrm{eV} \\ \text { 340. a } 171 \mathrm{a} & 1.000 & -0.166671 \mathrm{H}= & -4.535 \mathrm{eV} \\ \text { 339. a } 170 \mathrm{a} & 1.000 & -0.166726 \mathrm{H}= & -4.537 \mathrm{eV} \\ \text { 338. b } 169 \mathrm{a} & 1.000 & -0.212587 \mathrm{H}= & -5.785 \mathrm{eV} \\ \text { 337. b } 168 \mathrm{a} & 1.000 & -0.212614 \mathrm{H}= & -5.786 \mathrm{eV} \\ \text { 336. a } 169 \mathrm{a} & 1.000 & -0.215195 \mathrm{H}= & -5.856 \mathrm{eV} \\ \text { 335. a } 168 \mathrm{a} & 1.000 & -0.215227 \mathrm{H}= & -5.857 \mathrm{eV} \\ \text { 334. b } 167 \mathrm{a} & 1.000 & -0.234963 \mathrm{H}= & -6.394 \mathrm{eV} \\ \text { 333. b } 166 \mathrm{a} & 1.000 & -0.235005 \mathrm{H}= & -6.395 \mathrm{eV} \\ \text { 332. a } 167 \mathrm{a} & 1.000 & -0.239145 \mathrm{H}= & -6.507 \mathrm{eV} \\ \text { 331. a } 166 \mathrm{a} & 1.000 & -0.239189 \mathrm{H}= & -6.509 \mathrm{eV} \\ \text { 330. b } 165 \mathrm{a} & 1.000 & -0.285423 \mathrm{H}= & -7.767 \mathrm{eV} \\ \text { 329. b } 164 \mathrm{a} & 1.000 & -0.285446 \mathrm{H}= & -7.767 \mathrm{eV} \\ \text { 328. a } 165 \mathrm{a} & 1.000 & -0.285459 \mathrm{H}= & -7.768 \mathrm{eV} \\ \text { 327. a } 164 \mathrm{a} & 1.000 & -0.285482 \mathrm{H}= & -7.768 \mathrm{eV} \\ \text { 326. b } 163 \mathrm{a} & 1.000 & -0.285486 \mathrm{H}= & -7.768 \mathrm{eV}\end{array}$

\section{3 $\mathrm{Pu}$}

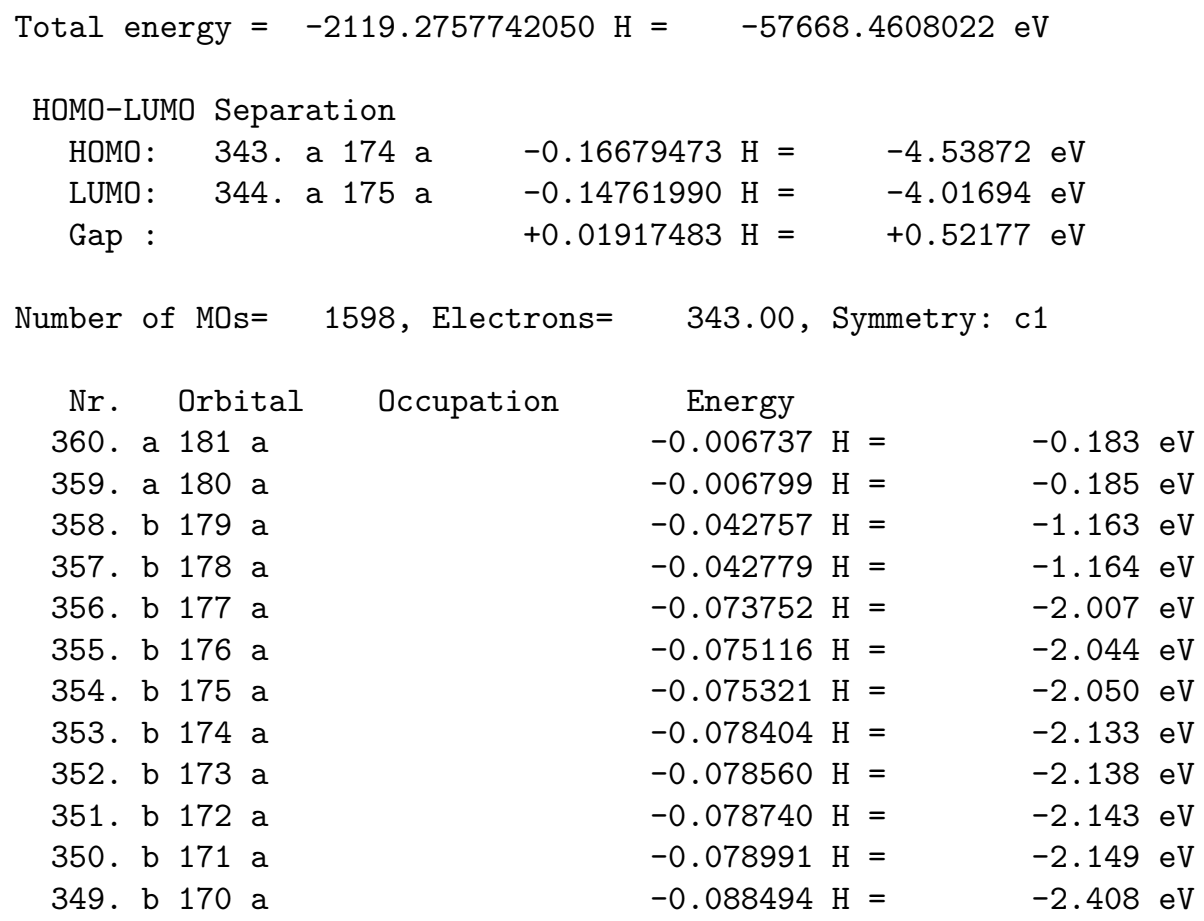




$\begin{array}{llll}\text { 348. a } 179 \text { a } & & -0.103142 \mathrm{H}= & -2.807 \mathrm{eV} \\ \text { 347. a } 178 \mathrm{a} & -0.104113 \mathrm{H}= & -2.833 \mathrm{eV} \\ \text { 346. a } 177 \mathrm{a} & -0.113859 \mathrm{H}= & -3.098 \mathrm{eV} \\ \text { 345. a } 176 \mathrm{a} & & -0.147598 \mathrm{H}= & -4.016 \mathrm{eV} \\ \text { 344. a } 175 \mathrm{a} & & -0.147620 \mathrm{H}= & -4.017 \mathrm{eV} \\ \text { 343. a } 174 \mathrm{a} & 1.000 & -0.166795 \mathrm{H}= & -4.539 \mathrm{eV} \\ \text { 342. a } 173 \mathrm{a} & 1.000 & -0.166798 \mathrm{H}= & -4.539 \mathrm{eV} \\ \text { 341. a } 172 \mathrm{a} & 1.000 & -0.186966 \mathrm{H}= & -5.088 \mathrm{eV} \\ \text { 340. a } 171 \mathrm{a} & 1.000 & -0.190592 \mathrm{H}= & -5.186 \mathrm{eV} \\ \text { 339. b } 169 \mathrm{a} & 1.000 & -0.212372 \mathrm{H}= & -5.779 \mathrm{eV} \\ \text { 338. b } 168 \mathrm{a} & 1.000 & -0.212397 \mathrm{H}= & -5.780 \mathrm{eV} \\ \text { 337. a } 170 \mathrm{a} & 1.000 & -0.213410 \mathrm{H}= & -5.807 \mathrm{eV} \\ \text { 336. a } 169 \mathrm{a} & 1.000 & -0.219066 \mathrm{H}= & -5.961 \mathrm{eV} \\ \text { 335. a } 168 \mathrm{a} & 1.000 & -0.219088 \mathrm{H}= & -5.962 \mathrm{eV} \\ \text { 334. b } 167 \mathrm{a} & 1.000 & -0.232419 \mathrm{H}= & -6.324 \mathrm{eV} \\ \text { 333. b } 166 \mathrm{a} & 1.000 & -0.232450 \mathrm{H}= & -6.325 \mathrm{eV} \\ \text { 332. a } 167 \mathrm{a} & 1.000 & -0.235222 \mathrm{H}= & -6.401 \mathrm{eV} \\ \text { 331. a } 166 \mathrm{a} & 1.000 & -0.235245 \mathrm{H}= & -6.401 \mathrm{eV} \\ \text { 330. a } 165 \mathrm{a} & 1.000 & -0.285144 \mathrm{H}= & -7.759 \mathrm{eV} \\ \text { 329. a } 164 \mathrm{a} & 1.000 & -0.285185 \mathrm{H}= & -7.760 \mathrm{eV} \\ \text { 328. b } 165 \mathrm{a} & 1.000 & -0.285227 \mathrm{H}= & -7.761 \mathrm{eV}\end{array}$

\subsection{Am}

\begin{tabular}{|c|c|c|c|c|c|c|c|}
\hline \multicolumn{8}{|c|}{ HOMO-LUMO Separation } \\
\hline HOMO : & $344 . \mathrm{k}$ & b $175 \mathrm{a}$ & \multicolumn{2}{|c|}{$-0.18130873 \mathrm{H}=$} & \multicolumn{3}{|c|}{$-4.93366 \mathrm{eV}$} \\
\hline LUMO: & $345 . \mathrm{k}$ & b 176 a & \multicolumn{2}{|c|}{$-0.17478106 \mathrm{H}=$} & \multicolumn{3}{|c|}{$-4.75604 \mathrm{eV}$} \\
\hline Gap : & & & \multicolumn{2}{|c|}{$+0.00652767 \mathrm{H}=$} & \multicolumn{3}{|c|}{$+0.17763 \mathrm{eV}$} \\
\hline Number of & $\mathrm{MOs}=$ & 1598 & trons $=$ & 344.00 & Symm & $c 1$ & \\
\hline $\mathrm{Nr}$. & Orbital & $\mathrm{Occl}$ & tion & Energy & & & \\
\hline 361. a 1 & $180 \mathrm{a}$ & & & -0.001428 & $\mathrm{H}=$ & -0.039 & \\
\hline 360. b 1 & $181 \mathrm{a}$ & & & -0.010028 & $\mathrm{H}=$ & -0.273 & $\mathrm{eV}$ \\
\hline 359. b 1 & $180 \mathrm{a}$ & & & -0.010038 & $\mathrm{H}=$ & -0.273 & $\mathrm{eV}$ \\
\hline 358. a 1 & 179 a & & & -0.054542 & $\mathrm{H}=$ & -1.484 & $\mathrm{eV}$ \\
\hline 357. a 1 & 178 a & & & -0.064005 & $\mathrm{H}=$ & -1.742 & \\
\hline 356. a 1 & 177 a & & & -0.065352 & $\mathrm{H}=$ & -1.778 & $\mathrm{eV}$ \\
\hline 355. a 1 & 176 a & & & -0.067531 & $\mathrm{H}=$ & -1.838 & $\mathrm{eV}$ \\
\hline 354. a 1 & 175 a & & & -0.077050 & $\mathrm{H}=$ & -2.097 & $\mathrm{eV}$ \\
\hline 353. a 1 & 174 a & & & -0.077954 & $\mathrm{H}=$ & -2.121 & $\mathrm{eV}$ \\
\hline 352. a 1 & 173 a & & & -0.078891 & $\mathrm{H}=$ & -2.147 & eV \\
\hline 351. a 1 & 172 a & & & -0.079376 & $\mathrm{H}=$ & -2.160 & \\
\hline 350. a 1 & 171 a & & & -0.081670 & $\mathrm{H}=$ & -2.222 & \\
\hline 349. a 1 & $170 \mathrm{a}$ & & & -0.091259 & $\mathrm{H}=$ & -2.483 & eV \\
\hline 348. b 1 & 179 a & & & -0.103907 & $\mathrm{H}=$ & -2.827 & $\mathrm{eV}$ \\
\hline 347. b 1 & 178 a & & & -0.104263 & $\mathrm{H}=$ & -2.837 & $\mathrm{eV}$ \\
\hline
\end{tabular}




$\begin{array}{llll}\text { 346. b } 177 \mathrm{a} & & -0.119022 \mathrm{H}= & -3.239 \mathrm{eV} \\ \text { 345. b } 176 \mathrm{a} & & -0.174781 \mathrm{H}= & -4.756 \mathrm{eV} \\ \text { 344. b } 175 \mathrm{a} & 0.995 & -0.181309 \mathrm{H}= & -4.934 \mathrm{eV} \\ \text { 343. b } 174 \mathrm{a} & 1.000 & -0.206912 \mathrm{H}= & -5.630 \mathrm{eV} \\ \text { 342. b } 173 \mathrm{a} & 1.000 & -0.210779 \mathrm{H}= & -5.736 \mathrm{eV} \\ \text { 341. b } 172 \mathrm{a} & 1.000 & -0.210881 \mathrm{H}= & -5.738 \mathrm{eV} \\ \text { 340. a } 169 \mathrm{a} & 1.000 & -0.211863 \mathrm{H}= & -5.765 \mathrm{eV} \\ \text { 339. a } 168 \mathrm{a} & 1.000 & -0.213923 \mathrm{H}= & -5.821 \mathrm{eV} \\ \text { 338. b } 171 \mathrm{a} & 1.000 & -0.215741 \mathrm{H}= & -5.871 \mathrm{eV} \\ \text { 337. b } 170 \mathrm{a} & 1.000 & -0.227287 \mathrm{H}= & -6.185 \mathrm{eV} \\ \text { 336. b } 169 \mathrm{a} & 1.000 & -0.228112 \mathrm{H}= & -6.207 \mathrm{eV} \\ \text { 335. a } 167 \mathrm{a} & 1.000 & -0.228680 \mathrm{H}= & -6.223 \mathrm{eV} \\ \text { 334. b } 168 \mathrm{a} & 1.000 & -0.229610 \mathrm{H}= & -6.248 \mathrm{eV} \\ \text { 333. b } 167 \mathrm{a} & 1.000 & -0.230832 \mathrm{H}= & -6.281 \mathrm{eV} \\ \text { 332. a } 166 \mathrm{a} & 1.000 & -0.231123 \mathrm{H}= & -6.289 \mathrm{eV} \\ \text { 331. b } 166 \mathrm{a} & 1.000 & -0.233249 \mathrm{H}= & -6.347 \mathrm{eV} \\ \text { 330. b } 165 \mathrm{a} & 1.000 & -0.284860 \mathrm{H}= & -7.751 \mathrm{eV} \\ \text { 329. b } 164 \mathrm{a} & 1.000 & -0.284870 \mathrm{H}= & -7.752 \mathrm{eV}\end{array}$

\section{$6.5 \mathrm{Bk}$}

\begin{tabular}{|c|c|c|c|c|c|c|}
\hline \multicolumn{7}{|c|}{ HOMO-LUMO Separation } \\
\hline HOMO: & $346 . k$ & b 170 a & \multicolumn{2}{|c|}{$-0.18903650 \mathrm{H}=$} & \multicolumn{2}{|c|}{$-5.14395 \mathrm{eV}$} \\
\hline LUMO: & $347 . \mathrm{k}$ & b 171 a & \multicolumn{2}{|c|}{$-0.16129495 \mathrm{H}=$} & \multicolumn{2}{|c|}{$-4.38906 \mathrm{eV}$} \\
\hline & & & \multicolumn{2}{|c|}{$+0.02774156 \mathrm{H}=$} & \multicolumn{2}{|c|}{$+0.75489 \mathrm{eV}$} \\
\hline Number of & $\mathrm{MOs}=$ & $1598, \mathrm{E}$ & ctrons $=$ & 346.00 & Symm & c1 \\
\hline $\mathrm{Nr}$. & Orbital & $\mathrm{Occu}$ & tion & Energy & & \\
\hline 363. a 1 & $182 \mathrm{a}$ & & & +0.004017 & $\mathrm{H}=$ & $+0.109 \mathrm{e}$ \\
\hline 362. b 1 & $181 \mathrm{a}$ & & & -0.010510 & $\mathrm{H}=$ & $-0.286 \mathrm{e}$ \\
\hline $361 . \mathrm{b} 1$ & $180 \mathrm{a}$ & & & -0.010626 & $\mathrm{H}=$ & $-0.289 \mathrm{e}$ \\
\hline 360. a 1 & $181 \mathrm{a}$ & & & -0.018235 & $\mathrm{H}=$ & -0.496 e \\
\hline 359. a 1 & $180 \mathrm{a}$ & & & -0.018392 & $\mathrm{H}=$ & -0.500 e \\
\hline 358. b 1 & 179 a & & & -0.083151 & $\mathrm{H}=$ & $-2.263 \mathrm{e}$ \\
\hline $357 . \mathrm{b} 1$ & 178 a & & & -0.083489 & $\mathrm{H}=$ & $-2.272 \mathrm{e}$ \\
\hline 356. a 1 & 179 a & & & -0.101738 & $\mathrm{H}=$ & -2.768 e \\
\hline 355. b 1 & $177 \mathrm{a}$ & & & -0.102005 & $\mathrm{H}=$ & -2.776 e \\
\hline 354. a 1 & 178 a & & & -0.102094 & $\mathrm{H}=$ & $-2.778 \mathrm{e}$ \\
\hline 353. b 1 & 176 a & & & -0.117623 & $\mathrm{H}=$ & $-3.201 \mathrm{e}$ \\
\hline 352. a 1 & 177 a & & & -0.122717 & $\mathrm{H}=$ & $-3.339 e$ \\
\hline $351 . \mathrm{b} 1$ & 175 a & & & -0.156939 & $\mathrm{H}=$ & $-4.271 \mathrm{e}$ \\
\hline 350. b 1 & $174 \mathrm{a}$ & & & -0.158068 & $\mathrm{H}=$ & $-4.301 \mathrm{e}$ \\
\hline 349. b 1 & 173 a & & & -0.159887 & $\mathrm{H}=$ & $-4.351 \mathrm{e}$ \\
\hline 348. b 1 & 172 a & & & -0.160214 & $\mathrm{H}=$ & $-4.360 \mathrm{e}$ \\
\hline $347 . \quad b \quad 1$ & $171 \mathrm{a}$ & & & -0.161295 & $\mathrm{H}=$ & $-4.389 \mathrm{e}$ \\
\hline 346. b 1 & $170 \mathrm{a}$ & & & -0.189037 & $\mathrm{H}=$ & $-5.144 \mathrm{e}$ \\
\hline
\end{tabular}




$\begin{array}{lllll}\text { 345. a } 176 \mathrm{a} & 1.000 & -0.206680 \mathrm{H}= & -5.624 \mathrm{eV} \\ \text { 344. a } 175 \mathrm{a} & 1.000 & -0.206744 \mathrm{H}= & -5.626 \mathrm{eV} \\ \text { 343. b } 169 \mathrm{a} & 1.000 & -0.218786 \mathrm{H}= & -5.953 \mathrm{eV} \\ \text { 342. b } 168 \mathrm{a} & 1.000 & -0.218848 \mathrm{H}= & -5.955 \mathrm{eV} \\ \text { 341. b } 167 \mathrm{a} & 1.000 & -0.229388 \mathrm{H}= & -6.242 \mathrm{eV} \\ \text { 340. b } 166 \mathrm{a} & 1.000 & -0.229410 \mathrm{H}= & -6.243 \mathrm{eV} \\ \text { 339. a } 174 \mathrm{a} & 1.000 & -0.236694 \mathrm{H}= & -6.441 \mathrm{eV} \\ \text { 338. a } 173 \mathrm{a} & 1.000 & -0.236724 \mathrm{H}= & -6.442 \mathrm{eV} \\ \text { 337. a } 172 \mathrm{a} & 1.000 & -0.255797 \mathrm{H}= & -6.961 \mathrm{eV} \\ \text { 336. a } 171 \mathrm{a} & 1.000 & -0.281640 \mathrm{H}= & -7.664 \mathrm{eV} \\ \text { 335. a } 170 \mathrm{a} & 1.000 & -0.284962 \mathrm{H}= & -7.754 \mathrm{eV} \\ \text { 334. b } 165 \mathrm{a} & 1.000 & -0.285370 \mathrm{H}= & -7.765 \mathrm{eV} \\ \text { 333. b } 164 \mathrm{a} & 1.000 & -0.285399 \mathrm{H}= & -7.766 \mathrm{eV} \\ \text { 332. b } 163 \mathrm{a} & 1.000 & -0.285427 \mathrm{H}= & -7.767 \mathrm{eV} \\ \text { 331. b } 162 \mathrm{a} & 1.000 & -0.285467 \mathrm{H}= & -7.768 \mathrm{eV}\end{array}$

\subsection{No}

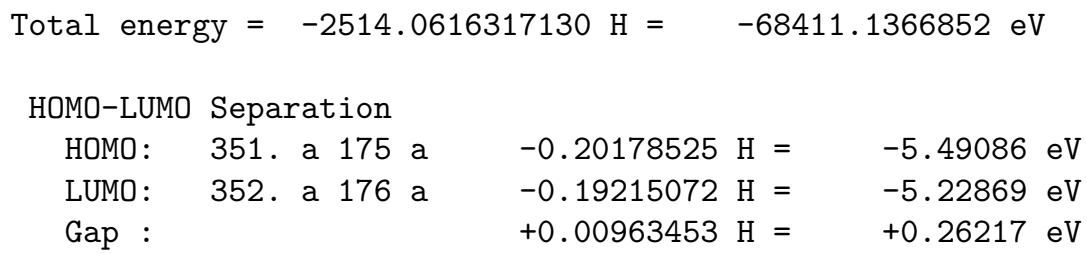

\begin{tabular}{|c|c|c|c|c|}
\hline $\mathrm{Nr}$. & Orbital & Occupation & Energy & \\
\hline 368. a & $184 \mathrm{a}$ & & $+0.018239 \mathrm{H}=$ & $+0.496 \mathrm{eV}$ \\
\hline $367 . \mathrm{b}$ & $184 a$ & & $+0.018030 \mathrm{H}=$ & $+0.491 \mathrm{eV}$ \\
\hline 366. a & $183 \mathrm{a}$ & & $+0.008426 \mathrm{H}=$ & $+0.229 \mathrm{eV}$ \\
\hline 365. a & $182 \mathrm{a}$ & & $+0.008140 \mathrm{H}=$ & $+0.222 \mathrm{eV}$ \\
\hline $364 . \mathrm{b}$ & 183 a & & $+0.008078 \mathrm{H}=$ & $+0.220 \mathrm{eV}$ \\
\hline 363. b & $182 \mathrm{a}$ & & $+0.006597 \mathrm{H}=$ & $+0.180 \mathrm{eV}$ \\
\hline 362. a & $181 \mathrm{a}$ & & $-0.011310 \mathrm{H}=$ & $-0.308 \mathrm{eV}$ \\
\hline 361 a & $180 \mathrm{a}$ & & $-0.011350 \mathrm{H}=$ & $-0.309 \mathrm{eV}$ \\
\hline $360 . \mathrm{b}$ & $181 \mathrm{a}$ & & $-0.019768 \mathrm{H}=$ & $-0.538 \mathrm{eV}$ \\
\hline $359 . \mathrm{b}$ & $180 \mathrm{a}$ & & $-0.019828 \mathrm{H}=$ & $-0.540 \mathrm{eV}$ \\
\hline 358. a & 179 a & & $-0.067155 \mathrm{H}=$ & $-1.827 \mathrm{eV}$ \\
\hline $357 . a$ & 178 a & & $-0.067390 \mathrm{H}=$ & $-1.834 \mathrm{eV}$ \\
\hline $356 . \mathrm{b}$ & 179 a & & $-0.071731 \mathrm{H}=$ & $-1.952 \mathrm{eV}$ \\
\hline $355 . \mathrm{b}$ & $178 \mathrm{a}$ & & $-0.071958 \mathrm{H}=$ & $-1.958 \mathrm{eV}$ \\
\hline 354. a & 177 a & & $-0.098937 \mathrm{H}=$ & $-2.692 \mathrm{eV}$ \\
\hline 353. b & 177 a & & $-0.101458 \mathrm{H}=$ & $-2.761 \mathrm{eV}$ \\
\hline 352. a & 176 a & & $-0.192151 \mathrm{H}=$ & $-5.229 \mathrm{eV}$ \\
\hline 351 . a & $175 \mathrm{a}$ & 1.000 & $-0.201785 \mathrm{H}=$ & $-5.491 \mathrm{eV}$ \\
\hline $350 . \mathrm{b}$ & 176 a & 1.000 & $-0.204296 \mathrm{H}=$ & $-5.559 \mathrm{eV}$ \\
\hline $349 . \mathrm{b}$ & 175 a & 1.000 & $-0.207854 \mathrm{H}=$ & $-5.656 \mathrm{eV}$ \\
\hline 348. a & $174 \mathrm{a}$ & 1.000 & $-0.216839 \mathrm{H}=$ & $-5.900 \mathrm{eV}$ \\
\hline
\end{tabular}




$\begin{array}{llll}\text { 347. a } 173 \mathrm{a} & 1.000 & -0.226919 \mathrm{H}= & -6.175 \mathrm{eV} \\ \text { 346. b } 174 \mathrm{a} & 1.000 & -0.228828 \mathrm{H}= & -6.227 \mathrm{eV} \\ \text { 345. b } 173 \mathrm{a} & 1.000 & -0.230700 \mathrm{H}= & -6.278 \mathrm{eV} \\ \text { 344. a } 172 \mathrm{a} & 1.000 & -0.238210 \mathrm{H}= & -6.482 \mathrm{eV} \\ \text { 343. b } 172 \mathrm{a} & 1.000 & -0.240973 \mathrm{H}= & -6.557 \mathrm{eV} \\ \text { 342. a } 171 \mathrm{a} & 1.000 & -0.243087 \mathrm{H}= & -6.615 \mathrm{eV} \\ \text { 341. a } 170 \mathrm{a} & 1.000 & -0.243444 \mathrm{H}= & -6.624 \mathrm{eV} \\ \text { 340. a } 169 \mathrm{a} & 1.000 & -0.243946 \mathrm{H}= & -6.638 \mathrm{eV} \\ \text { 339. a } 168 \mathrm{a} & 1.000 & -0.245279 \mathrm{H}= & -6.674 \mathrm{eV} \\ \text { 338. b } 171 \mathrm{a} & 1.000 & -0.245543 \mathrm{H}= & -6.682 \mathrm{eV} \\ \text { 337. a } 167 \mathrm{a} & 1.000 & -0.246021 \mathrm{H}= & -6.695 \mathrm{eV} \\ \text { 336. b } 170 \mathrm{a} & 1.000 & -0.246310 \mathrm{H}= & -6.702 \mathrm{eV}\end{array}$

\section{7 $\mathrm{Lr}$}

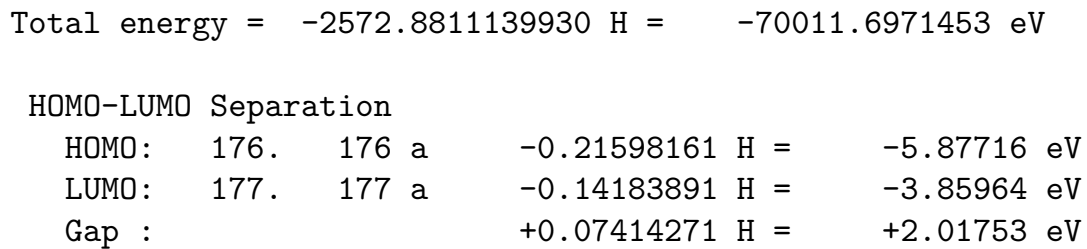

\begin{tabular}{|c|c|c|c|c|}
\hline $\mathrm{Nr}$. & Orbital & Occupation & Energy & \\
\hline 193. & $193 \mathrm{a}$ & & $+0.044306 \mathrm{H}=$ & $+1.206 \mathrm{eV}$ \\
\hline 192. & 192 a & & $+0.041546 \mathrm{H}=$ & $+1.131 \mathrm{eV}$ \\
\hline 191. & $191 \mathrm{a}$ & & $+0.041268 \mathrm{H}=$ & $+1.123 \mathrm{eV}$ \\
\hline 190. & $190 \mathrm{a}$ & & $+0.038318 \mathrm{H}=$ & $+1.043 \mathrm{eV}$ \\
\hline 189. & 189 a & & $+0.028080 \mathrm{H}=$ & $+0.764 \mathrm{eV}$ \\
\hline 188. & $188 \mathrm{a}$ & & $+0.027970 \mathrm{H}=$ & $+0.761 \mathrm{eV}$ \\
\hline 187. & $187 \mathrm{a}$ & & $+0.026015 \mathrm{H}=$ & $+0.708 \mathrm{eV}$ \\
\hline 186. & $186 \mathrm{a}$ & & $+0.025948 \mathrm{H}=$ & $+0.706 \mathrm{eV}$ \\
\hline 185. & $185 \mathrm{a}$ & & $+0.020480 \mathrm{H}=$ & $+0.557 \mathrm{eV}$ \\
\hline 184. & $184 \mathrm{a}$ & & $+0.016603 \mathrm{H}=$ & $+0.452 \mathrm{eV}$ \\
\hline 183. & $183 \mathrm{a}$ & & $+0.003701 \mathrm{H}=$ & $+0.101 \mathrm{eV}$ \\
\hline 182. & $182 \mathrm{a}$ & & $+0.003146 \mathrm{H}=$ & $+0.086 \mathrm{eV}$ \\
\hline 181. & $181 \mathrm{a}$ & & $-0.024461 \mathrm{H}=$ & $-0.666 \mathrm{eV}$ \\
\hline 180. & $180 \mathrm{a}$ & & $-0.024484 \mathrm{H}=$ & $-0.666 \mathrm{eV}$ \\
\hline 179. & 179 a & & $-0.114286 \mathrm{H}=$ & $-3.110 \mathrm{eV}$ \\
\hline 178. & $178 \mathrm{a}$ & & $-0.114505 \mathrm{H}=$ & $-3.116 \mathrm{eV}$ \\
\hline 177. & $177 \mathrm{a}$ & & $-0.141839 \mathrm{H}=$ & $-3.860 \mathrm{eV}$ \\
\hline 176. & $176 \mathrm{a}$ & 2.000 & $-0.215982 \mathrm{H}=$ & $-5.877 \mathrm{eV}$ \\
\hline 175 . & $175 \mathrm{a}$ & 2.000 & $-0.215993 \mathrm{H}=$ & $-5.877 \mathrm{eV}$ \\
\hline 174. & $174 \mathrm{a}$ & 2.000 & $-0.240987 \mathrm{H}=$ & $-6.558 \mathrm{eV}$ \\
\hline 173. & $173 \mathrm{a}$ & 2.000 & $-0.241011 \mathrm{H}=$ & $-6.558 \mathrm{eV}$ \\
\hline 172. & $172 \mathrm{a}$ & 2.000 & $-0.286334 \mathrm{H}=$ & $-7.792 \mathrm{eV}$ \\
\hline 171. & $171 \mathrm{a}$ & 2.000 & $-0.286360 \mathrm{H}=$ & $-7.792 \mathrm{eV}$ \\
\hline 170. & $170 \mathrm{a}$ & 2.000 & $-0.286547 \mathrm{H}=$ & $-7.797 \mathrm{eV}$ \\
\hline
\end{tabular}




$\begin{array}{lllll}\text { 169. } & 169 \mathrm{a} & 2.000 & -0.286584 \mathrm{H}= & -7.798 \mathrm{eV} \\ \text { 168. } & 168 \mathrm{a} & 2.000 & -0.303063 \mathrm{H}= & -8.247 \mathrm{eV} \\ 167 . & 167 \mathrm{a} & 2.000 & -0.311298 \mathrm{H}= & -8.471 \mathrm{eV} \\ 166 . & 166 \mathrm{a} & 2.000 & -0.311319 \mathrm{H}= & -8.471 \mathrm{eV} \\ 165 . & 165 \mathrm{a} & 2.000 & -0.312233 \mathrm{H}= & -8.496 \mathrm{eV} \\ 164 . & 164 \mathrm{a} & 2.000 & -0.312258 \mathrm{H}= & -8.497 \mathrm{eV} \\ 163 . & 163 \mathrm{a} & 2.000 & -0.321291 \mathrm{H}= & -8.743 \mathrm{eV} \\ 162 . & 162 \mathrm{a} & 2.000 & -0.321353 \mathrm{H}= & -8.744 \mathrm{eV} \\ 161 . & 161 \mathrm{a} & 2.000 & -0.324124 \mathrm{H}= & -8.820 \mathrm{eV}\end{array}$

\section{Optimized coordinates for solvated $\left[\mathrm{Th}\left(\mathrm{Cp}^{\mathrm{iPr}}\right)_{2}\right]^{-}$anion}

$\begin{array}{rr}\text { \$coord } & \\ -0.24521441010128 & 2.30581154452109 \\ -0.64969174746909 & 5.13960491241571 \\ 0.13083096452823 & 5.97169705833205 \\ 0.49074377538192 & 6.92812843348046 \\ -2.26657147984179 & 0.46983164877178 \\ -5.08639312277189 & 0.96421987696401 \\ -5.63967497293186 & 1.94556778318510 \\ -6.42892312121624 & 2.62379130438479 \\ -1.13913518806483 & -2.01913770755442 \\ -2.48212816330890 & -4.54827199385665 \\ -3.61578414787073 & -4.76888980659859 \\ -4.45532084929443 & -5.31235462006172 \\ 1.57109161468273 & -1.71563946426389 \\ 3.56329874102217 & -3.76983916012270 \\ 3.41211544834397 & -4.90095166204520 \\ 3.69425868044113 & -5.88917504067068 \\ 2.11688856201447 & 0.95046229105626 \\ 4.69069569299315 & 2.21361676379692 \\ 5.75531052962061 & 1.73772587547099 \\ 6.71945595737236 & 1.67008768866365 \\ -2.70980178356285 & 5.43731071982020 \\ -0.65233324310656 & 4.66632458188342 \\ -0.57101875475413 & 7.90596937487125 \\ 2.21021286070643 & 5.99413626399199 \\ -0.12923138481215 & 8.90610832888602 \\ 2.58340798362446 & 6.90389012386991 \\ -0.12514216014011 & 6.33109190212888 \\ -6.01206550228327 & -0.89918612041207 \\ -4.64315751689668 & 0.78734278417025 \\ -7.69695725314003 & 1.87568855319350 \\ -5.01659349174121 & 3.92793792353713 \\ -8.50236366886470 & 2.64247564923934 \\ -5.75841453063770 & 4.60579776145213 \\ -6.04763862451250 & 1.87636700016403 \\ -0.99466959579325 & -6.00474480224333 \\ -2.22272648617585 & -4.17703593633713\end{array}$

\begin{tabular}{ll}
4.76283636249069 & $\mathrm{c}$ \\
5.19083593149441 & $\mathrm{c}$ \\
7.88696878809956 & $\mathrm{c}$ \\
3.18533720244653 & $\mathrm{c}$ \\
4.73660097276645 & $\mathrm{c}$ \\
5.16264056519457 & $\mathrm{c}$ \\
7.86542589067008 & $\mathrm{c}$ \\
3.17081748310097 & $\mathrm{c}$ \\
4.75115890348079 & $\mathrm{c}$ \\
5.16719003189652 & $\mathrm{c}$ \\
7.85805635026974 & $\mathrm{c}$ \\
3.15554137272994 & $\mathrm{c}$ \\
4.78449051387754 & $\mathrm{c}$ \\
5.21486562758525 & $\mathrm{c}$ \\
7.91068846457155 & $\mathrm{c}$ \\
3.21137754843476 & $\mathrm{c}$ \\
4.79717792759773 & $\mathrm{c}$ \\
5.18945538224369 & $\mathrm{c}$ \\
7.87440659544686 & $\mathrm{c}$ \\
3.16169033639576 & $\mathrm{c}$ \\
5.07091562625885 & $\mathrm{~h}$ \\
9.32249841843703 & $\mathrm{~h}$ \\
8.30092637848735 & $\mathrm{~h}$ \\
8.11763623429763 & $\mathrm{~h}$ \\
3.53280687349342 & $\mathrm{~h}$ \\
3.19813181605403 & $\mathrm{~h}$ \\
1.27449429454462 & $\mathrm{~h}$ \\
5.02753668776428 & $\mathrm{~h}$ \\
9.29472549439377 & $\mathrm{~h}$ \\
8.27625073064761 & $\mathrm{~h}$ \\
8.10923846128759 & $\mathrm{~h}$ \\
3.51329870790906 & $\mathrm{~h}$ \\
\hline .20629724331323 & $\mathrm{~h}$ \\
\hline .30248872357920 & $\mathrm{~h}$
\end{tabular}
C c C h c c C C C C C $\mathrm{h}$ .

h

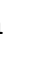

.

h

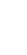

h

h

h




\begin{tabular}{|c|c|}
\hline-4.18700350065274 & -6.74766618822133 \\
\hline-5.31204733288961 & -3.56531980281919 \\
\hline-5.12944302898419 & -7.27226590900787 \\
\hline-6.12695426044518 & -4.05313357456629 \\
\hline-3.60092804668021 & -5.20270734193790 \\
\hline 5.40632978290344 & -2.80192691755061 \\
\hline 3.28479526533369 & -3.38420252701800 \\
\hline 5.11098093638497 & -6.06145821677002 \\
\hline 1.73656191184446 & -6.13291158956375 \\
\hline 5.36553136126149 & -7.11557222089996 \\
\hline 3.83306813365302 & -5.04852283599343 \\
\hline 1.99374350186200 & -7.10861899923894 \\
\hline 4.33766572827252 & 4.26483476325596 \\
\hline 7.38525202597395 & 3.00249972509623 \\
\hline 4.29122271594533 & 2.08960243502281 \\
\hline 6.41854566513999 & -0.23291372297033 \\
\hline 8.41784838619141 & 2.86176027569637 \\
\hline 5.95507394996335 & 2.07913358120624 \\
\hline 7.33051446163169 & -0.33077236637720 \\
\hline 0.00007276563380 & 0.00014959084804 \\
\hline 0.24509801591983 & -2.30578044247123 \\
\hline 0.64936994153737 & -5.13960689502030 \\
\hline-0.13146090424992 & -5.97171501383281 \\
\hline-0.49122180588459 & -6.92791966631808 \\
\hline 2.26656230653613 & -0.46990894273744 \\
\hline 5.08634159953423 & -0.96452087415681 \\
\hline 5.63948777001798 & -1.94631723110849 \\
\hline 6.42873660061245 & -2.62399997921578 \\
\hline 1.13924832760684 & 2.01911527523802 \\
\hline 2.48236226294650 & 4.54817688071021 \\
\hline 3.61621443530061 & 4.76867351805928 \\
\hline 4.45535617260255 & 5.31242407319475 \\
\hline-1.57098027348520 & 1.71576364417481 \\
\hline-3.56300136319921 & 3. 77012742594432 \\
\hline-3.41145270962064 & 4.90128300920750 \\
\hline-3.69363356686454 & 5.88952293155517 \\
\hline-2.11693155908701 & -0.95030829425557 \\
\hline-4.69081439614636 & -2.21338832846462 \\
\hline-5.75564870933066 & -1.73775800406698 \\
\hline-6.71943382512759 & -1.66992420554686 \\
\hline 2.70945593600982 & -5.43750209454452 \\
\hline 0.65176047898137 & -4.66653294085669 \\
\hline 0.57009984907286 & -7.90609879500586 \\
\hline-2.21086413335359 & -5.99388305725492 \\
\hline 0.12847023191898 & -8.90598942951531 \\
\hline-2.58388090495663 & -6.90338156582169 \\
\hline 0.12482328916060 & -6.33091990559388 \\
\hline 6.01217257948567 & 0.89882270962285 \\
\hline 4.64308853779106 & -0.78824897051630 \\
\hline 7.69677858285004 & -1.87671469606961 \\
\hline 5.01622325547367 & -3.92866871562992 \\
\hline
\end{tabular}

\begin{tabular}{|c|c|}
\hline 26346617334793 & \\
\hline 8.08206864442248 & \\
\hline 3.50019569121101 & \\
\hline 3.16381160221157 & \\
\hline 1.24688953534455 & \\
\hline 5.09906632121685 & \\
\hline 9.34658934213953 & \\
\hline 8. 32564041988704 & \\
\hline 8.13832525734883 & \\
\hline 3.55680541298791 & \\
\hline 1.29836053722606 & \\
\hline 3. 23085093698942 & \\
\hline 5.06687669137649 & \\
\hline 8.26004259256565 & \\
\hline 9.32713097133094 & \\
\hline 8.10632391989816 & \\
\hline 3.49347527923276 & \\
\hline 1.25611530139996 & \\
\hline 3.16636613054985 & \\
\hline-0.000037867 & \\
\hline 786464 & \\
\hline-5.190674240 & \\
\hline-7.88670559689638 & \\
\hline-3.18508796844789 & \\
\hline-4.73671432635365 & \\
\hline-5.16274293643920 & \\
\hline-7.86539085430085 & \\
\hline-3.1 & \\
\hline-4.75140874659368 & \\
\hline-5.16753124497862 & \\
\hline-7.85832940913068 & . \\
\hline-3.15574530059235 & \\
\hline-4.78467240341130 & \\
\hline-5.21512796188163 & c \\
\hline-7.91091681329232 & C \\
\hline-3.21168065995528 & c \\
\hline-4.79717541050071 & c \\
\hline-5.189241995 & c \\
\hline-7.87415521232872 & C \\
\hline-3.16131250229532 & \\
\hline-5.07084354918457 & II \\
\hline-9.32237332086177 & \\
\hline-8.30061477815976 & - \\
\hline-8.11718591490607 & \\
\hline-3.53253302948025 & \\
\hline-3.19779250593289 & \\
\hline-1.27429266223410 & \\
\hline-5.02787837503309 & \\
\hline-9.29489729701892 & \\
\hline-8.27620891563941 & \\
\hline -8 1088581 & \\
\hline
\end{tabular}




\begin{tabular}{rrrr}
8.50215449784723 & -2.64302891608873 & -3.51334849656510 & $\mathrm{~h}$ \\
5.75792864992089 & -4.60590768904261 & -3.20591707472318 & $\mathrm{~h}$ \\
6.04765810265435 & -1.87625514446343 & -1.25217869941889 & $\mathrm{~h}$ \\
0.99491095216005 & 6.00468438044655 & -5.04803356657354 & $\mathrm{~h}$ \\
2.22330113710351 & 4.17676505505517 & -9.30287969740931 & $\mathrm{~h}$ \\
4.18746348505808 & 6.74743885444790 & -8.26374398713060 & $\mathrm{~h}$ \\
5.31249942833372 & 3.56510623975045 & -8.08214431164431 & $\mathrm{~h}$ \\
5.12966348273855 & 7.27223433769352 & -3.50062061508104 & $\mathrm{~h}$ \\
6.12689295028109 & 4.05307558608136 & -3.16359956698792 & $\mathrm{~h}$ \\
3.60072338539958 & 5.20313696453974 & -1.24717244748801 & $\mathrm{~h}$ \\
-5.40617620243807 & 2.80248029586416 & -5.09940179691527 & $\mathrm{~h}$ \\
-3.28437253231714 & 3.38458248947631 & -9.34689082448626 & $\mathrm{~h}$ \\
-5.11005852809995 & 6.06216110418535 & -8.32588424015135 & $\mathrm{~h}$ \\
-1.73563511740636 & 6.13290965854271 & -8.13841508480631 & $\mathrm{~h}$ \\
-5.36458686388319 & 7.11630963737579 & -3.55727938970799 & $\mathrm{~h}$ \\
-3.83280534881085 & 5.04894466837086 & -1.29865095667978 & $\mathrm{~h}$ \\
-1.99281709005722 & 7.10855579230431 & -3.23100941807961 & $\mathrm{~h}$ \\
-4.33775670755468 & -4.26459579541084 & -5.06660037972151 & $\mathrm{~h}$ \\
-7.38558504393879 & -3.00261810983064 & -8.25952124050979 & $\mathrm{~h}$ \\
-4.29170242469724 & -2.08968947673729 & -9.32700917452904 & $\mathrm{~h}$ \\
-6.41896357888742 & 0.23283622953827 & -8.10618013147908 & $\mathrm{~h}$ \\
-8.41782021566386 & -2.86164115459327 & -3.49297364862419 & $\mathrm{~h}$ \\
-5.95489856622839 & -2.07897531318421 & -1.25579901126087 & $\mathrm{~h}$ \\
-7.33053390734931 & 0.33092089370000 & -3.16591158664367 & $\mathrm{~h}$ \\
\hline & & &
\end{tabular}
\$end

\section{Orbital occupations for solvated $\left[\mathbf{T h}\left(\mathbf{C p}^{\mathrm{iPr}{ }_{5}}\right)_{2}\right]^{-}$anion}

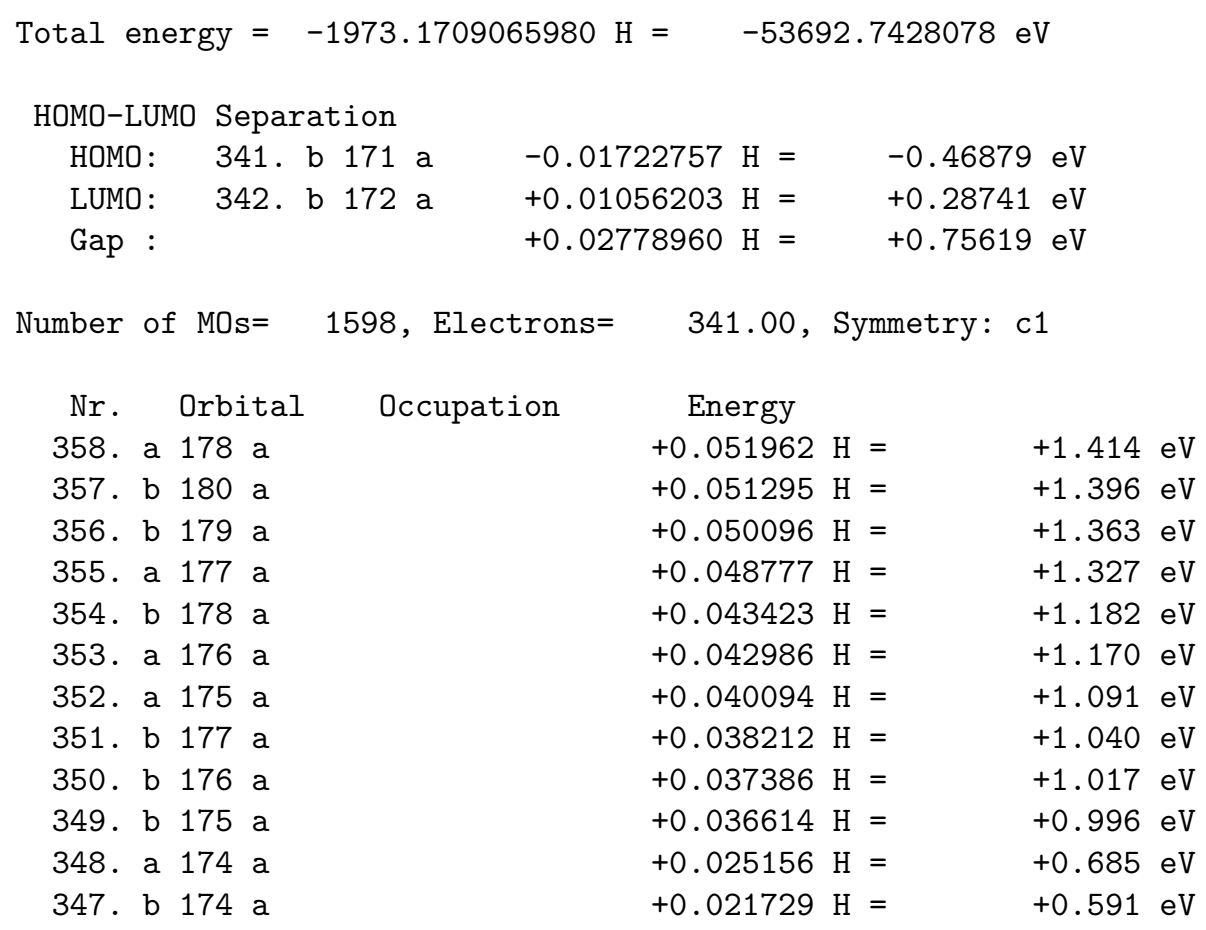


346. a 173 a

345. a 172 a

344. b 173 a

343. a 171 a

342. b 172 a

341. b 171 a

340. a 170 a

339. b 170 a

338. a 169 a

337. a 168 a

336. b 169 a

335. b 168 a

334. a 167 a

333. a 166 a

332. b 167 a

331. b 166 a

330. a 165 a

329. a 164 a

328. b 165 a

327. b 164 a

326. a 163 a
$+0.021625 \mathrm{H}=$

$+0.017811 \mathrm{H}=$

$+0.015925 \mathrm{H}=$

$+0.011077 \mathrm{H}=$

$+0.010562 \mathrm{H}=$

1.000

$-0.017228 \mathrm{H}=$

$-0.020483 \mathrm{H}=$

$-0.031301 \mathrm{H}=$

$-0.121126 \mathrm{H}=$

$-0.121304 \mathrm{H}=$

$-0.123168 \mathrm{H}=$

$-0.123394 \mathrm{H}=$

$-0.141442 \mathrm{H}=$

$-0.142050 \mathrm{H}=$

$-0.143883 \mathrm{H}=$

$-0.144975 \mathrm{H}=$

$-0.214958 \mathrm{H}=$

$-0.215106 \mathrm{H}=$

$-0.215118 \mathrm{H}=$

$-0.215260 \mathrm{H}=$

$-0.215687 \mathrm{H}=$

$$
\begin{aligned}
& +0.588 \mathrm{eV} \\
& +0.485 \mathrm{eV} \\
& +0.433 \mathrm{eV} \\
& +0.301 \mathrm{eV} \\
& +0.287 \mathrm{eV} \\
& -0.469 \mathrm{eV} \\
& -0.557 \mathrm{eV} \\
& -0.852 \mathrm{eV} \\
& -3.296 \mathrm{eV} \\
& -3.301 \mathrm{eV} \\
& -3.352 \mathrm{eV} \\
& -3.358 \mathrm{eV} \\
& -3.849 \mathrm{eV} \\
& -3.865 \mathrm{eV} \\
& -3.915 \mathrm{eV} \\
& -3.945 \mathrm{eV} \\
& -5.849 \mathrm{eV} \\
& -5.853 \mathrm{eV} \\
& -5.854 \mathrm{eV} \\
& -5.858 \mathrm{eV} \\
& -5.869 \mathrm{eV}
\end{aligned}
$$




\section{Natural Atomic Populations of HOMO for $\operatorname{An}\left(\mathrm{Cp}^{\mathrm{iPr}}\right)_{2}$}

\begin{tabular}{l|rrrr} 
& $\mathrm{s}$ & $\mathrm{p}$ & $\mathrm{d}$ & $\mathrm{f}$ \\
\hline Th & 0.351 & 0.000 & 0.409 & 0.000 \\
Am & 0.000 & 0.036 & 0.010 & 0.859 \\
No & 0.000 & 0.081 & 0.000 & 0.555 \\
Lr & 0.409 & 0.000 & 0.356 & 0.000 \\
\hline
\end{tabular}

Table 15: Natural atomic populations of the HOMO arising from the An atom for $\mathrm{An}\left(\mathrm{Cp}^{\mathrm{iPr}{ }_{5}}\right)_{2},(\mathrm{An}=\mathrm{Th}, \mathrm{Am}, \mathrm{No}, \mathrm{Lr})$. Those for $\mathrm{U}, \mathrm{Pu}$, and $\mathrm{Bk}$ are given in the manuscript. 